\title{
Meteorological Data for Four Sites at Surface-Disruption Features in Yucca Flat, Nevada Test Site, Nye County, Nevada, 1985-86
}

\author{
By R.L. Carman
}

U.S. GEOLOGICAL SURVEY

Open-File Report 94-491

Prepared in cooperation with the U.S. DEPARTMENT OF ENERGY

(Interagency Agreement DE-A108-91NV11040)

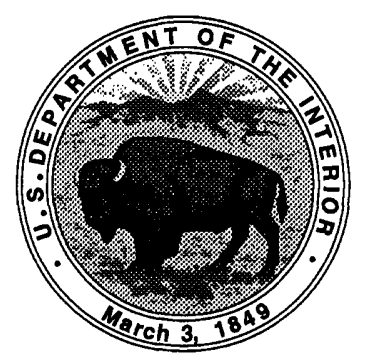




\section{U.S. DEPARTMENT OF THE INTERIOR BRUCE BABBITT, Secretary}

U.S. GEOLOGICAL SURVEY

GORDON P. EATON, Director

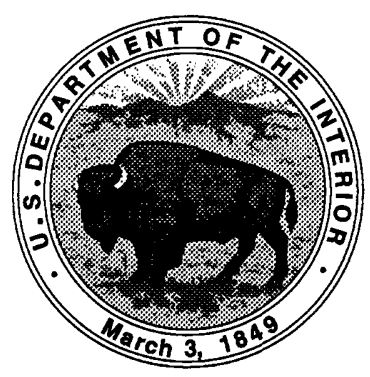

Any use of trade names in this publication is for descriptive purposes only and does not imply endorsement by the U.S. Government.

For additional information write to:

District Chief

U.S. Geological Survey 333 W. Nye Lane, Room 203 Carson City, NV 89706-0866
Copies of this report can be purchased from:

U.S. Geological Survey Earth Science Information Center Open-File Reports Section Box 25286, MS 517 Denver Federal Center Denver, CO 80225-0046 


\section{CONTENTS}

Abstract Introduction

\section{FIGURES}

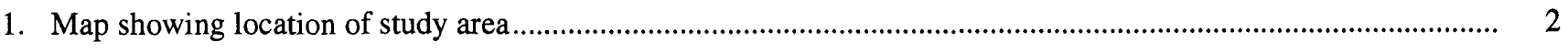

2. Sketch map showing location of instrument sites within study area .............................................................. 2

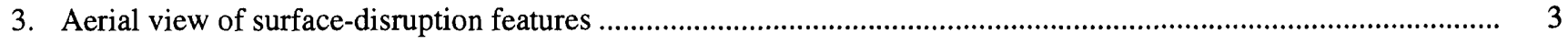

4. Photograph showing weather-station instruments ................................................................................................. 4

5. Photograph showing instruments for collecting bare-soil evaporation data....................................................... 5

\section{TABLES}

1-3. Bare-soil evaporation data:

1. Undisturbed-land-surface site, May 1985 through June 1986 ......................................................................... 6

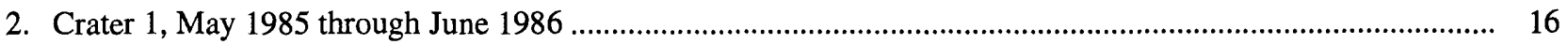

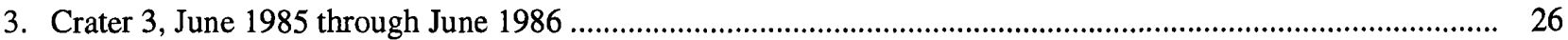

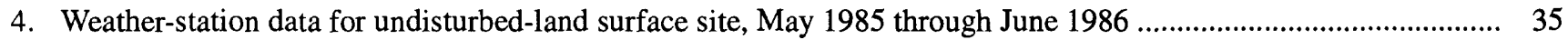

\section{CONVERSION FACTORS AND VERTICAL DATUM}

\begin{tabular}{rll}
\hline Multiply & By & To obtain \\
\hline centimeter & 0.3937 & inch \\
kilometer & 0.6214 & mile \\
meter & 3.281 & foot \\
meter per second & 2.237 & mile per hour \\
watt per square meter & 0.005287 & British thermal unit per minute per square foot \\
\hline
\end{tabular}

Temperature: Degrees Celsius $\left({ }^{\circ} \mathrm{C}\right)$ may be converted to degrees Fahrenheit $\left({ }^{\circ} \mathrm{F}\right)$ by using the formula ${ }^{\circ} \mathrm{F}=\left[1.8\left({ }^{\circ} \mathrm{C}\right)\right]+32$.

Sea Level: In this report, "sea level" refers to the National Geodetic Vertical Datum of 1929 (NGVD of 1929, formerly called SeaLevel Datum of 1929), which is derived from a general adjustment of the first-order leveling networks of the United States and Canada. 


\title{
Meteorological Data for Four Sites at Surface-Disruption Features in Yucca Flat, Nevada Test Site, Nye County, Nevada, 1985-86
}

\author{
By R.L. Carman
}

\section{Abstract}

Surface-disruption features, or craters, resulting from underground nuclear testing at the Nevada Test Site may increase the potential for ground-water recharge in an area that would normally produce little, if any, recharge. This report presents selected meteorological data resulting from a study of two surface-disruption features during May 1985 through June 1986. The data were collected at four adjacent sites in Yucca Flat, about 56 kilometers north of Mercury, Nev. Three sites (one in each of two craters and one at an undisturbed site at the original land surface) were instrumented to collect meteorological data for calculating bare-soil evaporation. These data include (1) long-wave radiation, (2) short-wave radiation, (3) net radiation, (4) air temperature, and (5) soil surface temperature. Meteorological data also were collected at a weather station at an undisturbed site near the study craters. Data collected at this site include (1) air temperature,

(2) relative humidity, (3) wind velocity, and

(4) wind direction.

\section{INTRODUCTION}

Since nuclear-weapons testing began at the Nevada Test Site in southern Nevada, questions have arisen regarding how the testing may affect natural physical processes in the testing areas. It is important to understand the changes that may occur, their effects, and future implications, if any.
One result of underground nuclear testing is the formation of surface-disruption features, or craters. Concern exists that the craters could increase the amount of infiltration and ground-water recharge in an area that normally would produce little, if any, recharge. Normally, precipitation in the area would be evaporated, taken up by plants, or held as soil moisture because of the low precipitation and high evaporation potential. The surface-disruption sites may increase the potential for ground-water recharge by acting as catchments for precipitation and overland flow. Such recharge could create problems from subsurface radionuclide migration.

A study was initiated in January 1985 to determine whether the surface-disruption features, which resulted from underground nuclear testing, are increasing the potential for ground-water recharge. This report presents the meteorological data collected during the study. By June 1985 bare-soil evaporation data (long- and short-wave radiation, air and soil surface temperature, net radiation) were being collected at three sites. One station was at an undisturbed site, and the other two stations were in the bottoms of craters. Data collection was discontinued after June 1986.

\section{STUDY SITE}

Two craters at the Nevada Test Site were chosen for study because of their proximity to each other and to Mercury, Nev., and because they appear to be representative of most craters in the area. The craters, referred to as craters 1 and 3, are in Yucca Flat, approximately 56 kilometers north of Mercury (figs. 1 and 2). 


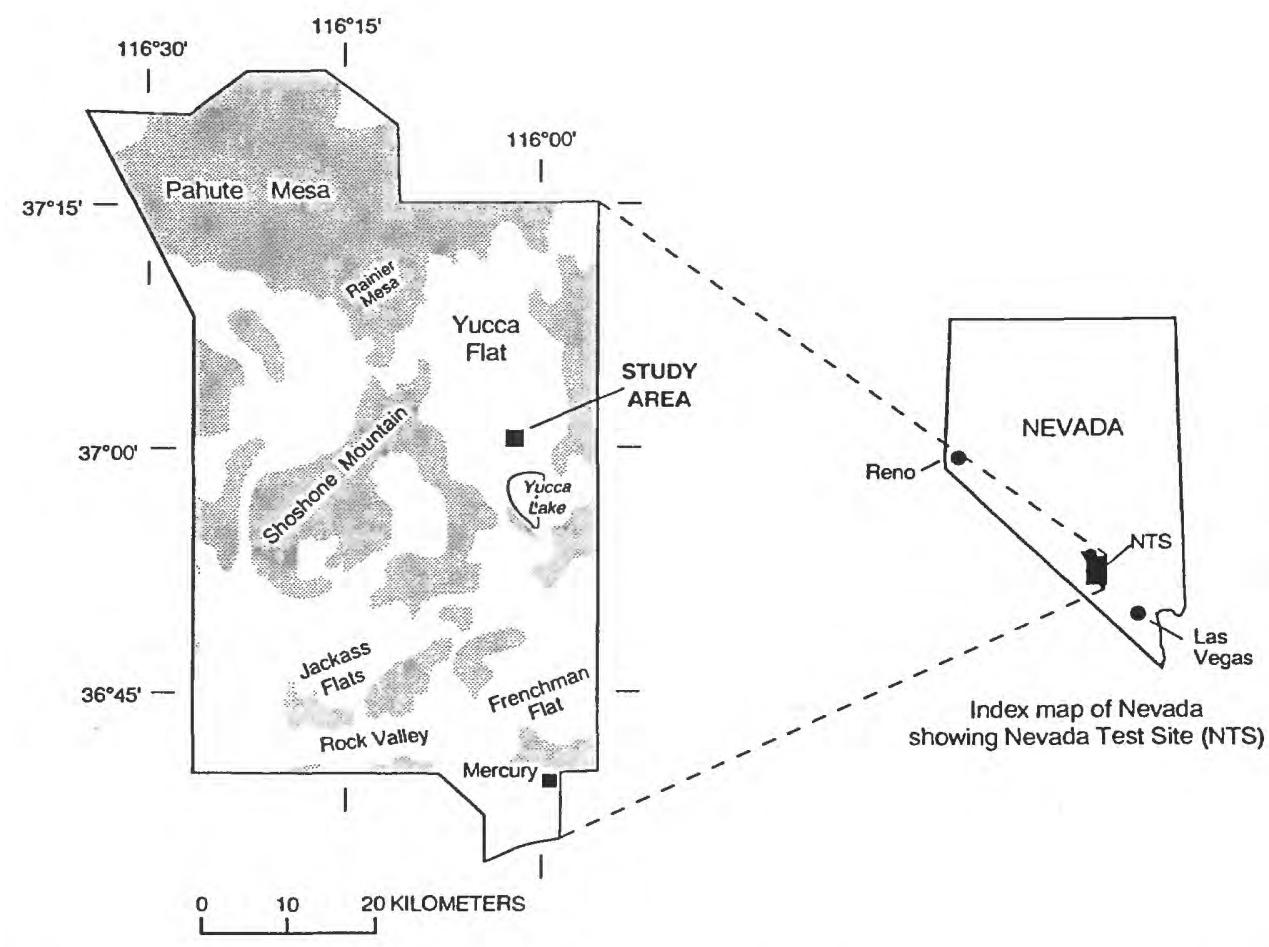

Figure 1. Location of study area within Nevada Test Site. Shading indicates higher altitude areas.

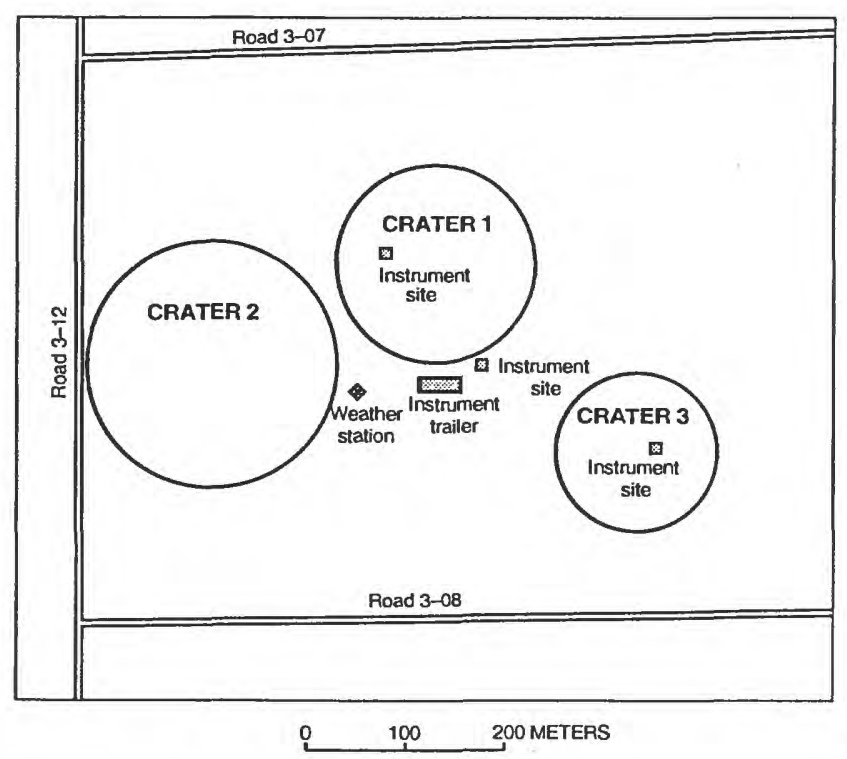

Figure 2. Location of U.S. Geological Survey instrument sites and weather station within study area (fig. 1).
Altitude of the undisturbed land surface is approximately 1,210 meters. The climate is arid. Precipitation in the area is 15 to 18 centimeters per year, and temperatures range from -10 to $41^{\circ} \mathrm{C}$. The area is underlain by alluvial deposits. The depth to water is about 460 meters.

Crater 1 is approximately 13 meters in depth and 190 meters in diameter; crater 3 is approximately 22 meters in depth and 153 meters in diameter (fig. 2). The craters exhibit extensive erosional features with deep gullies down the sides and depositional fans in the bottom. An aerial view of the craters at the Nevada Test Site is shown in figure 3. The study sites have sparse vegetation, consisting of desert lowland shrubs and grasses. In this study, vegetation was assumed to be negligible and the area was considered to be bare soil. 


\section{INSTRUMENTATION}

The weather-station instruments were erected at an undisturbed site near the southwest edge of crater 1 (fig. 2 ) and at a height of approximately 2 meters. Data on wind velocity and direction, relative humidity, and air temperature were collected. Instruments included a cup anemometer and wind vane for measuring wind velocity and direction and a relative-humidity probe with an attached thermistor (both enclosed in a fanaspirated radiation shield) for measuring relative humidity and air temperature (fig. 4A). The instruments were connected to a programmable-data-logger system that processed and recorded the data (fig. $4 B$ ). The sensors were scanned every 60 seconds and data values recorded. These values were integrated over a period of 60 minutes, and the hourly average value was recorded.

Meteorological data for calculating bare-soil evaporation were recorded continuously at three stations. One station was at an undisturbed site just outside the south edge of crater 1 ; the other two were at the bottoms of craters 1 and 3 (fig. 2). Data collected include incoming and outgoing short- and long-wave radiation, air temperature, soil surface temperature, and net radiation. The instruments were approximately 1 meter above the land surface. Instruments at the undisturbed site and in crater 1 included two precision pyranometers for measuring incoming and outgoing shortwave radiation, two precision pyrgeometers for measuring incoming and outgoing long-wave radiation, a thermistor psychrometer for measuring air temperature, a net radiometer for measuring net radiation, and an infrared thermometer for measuring soil surface temperature (fig. 5). The site in crater 3 was equipped with pyranometers, a thermistor psychrometer, and an infrared thermometer; it had no pyrgeometers or net radiometer. All the instruments from the stations were connected to a central data-logger system that processed and stored the data (fig. $4 B$ ). Measurements were taken every 60 seconds, the values integrated over 60 minutes, and the hourly average value recorded.

\section{DATA SUMMARY}

The daily meteorological data, summarized from the hourly field data, are presented in tables 1 through 3. The daily weather-station data are summarized in table 4 .

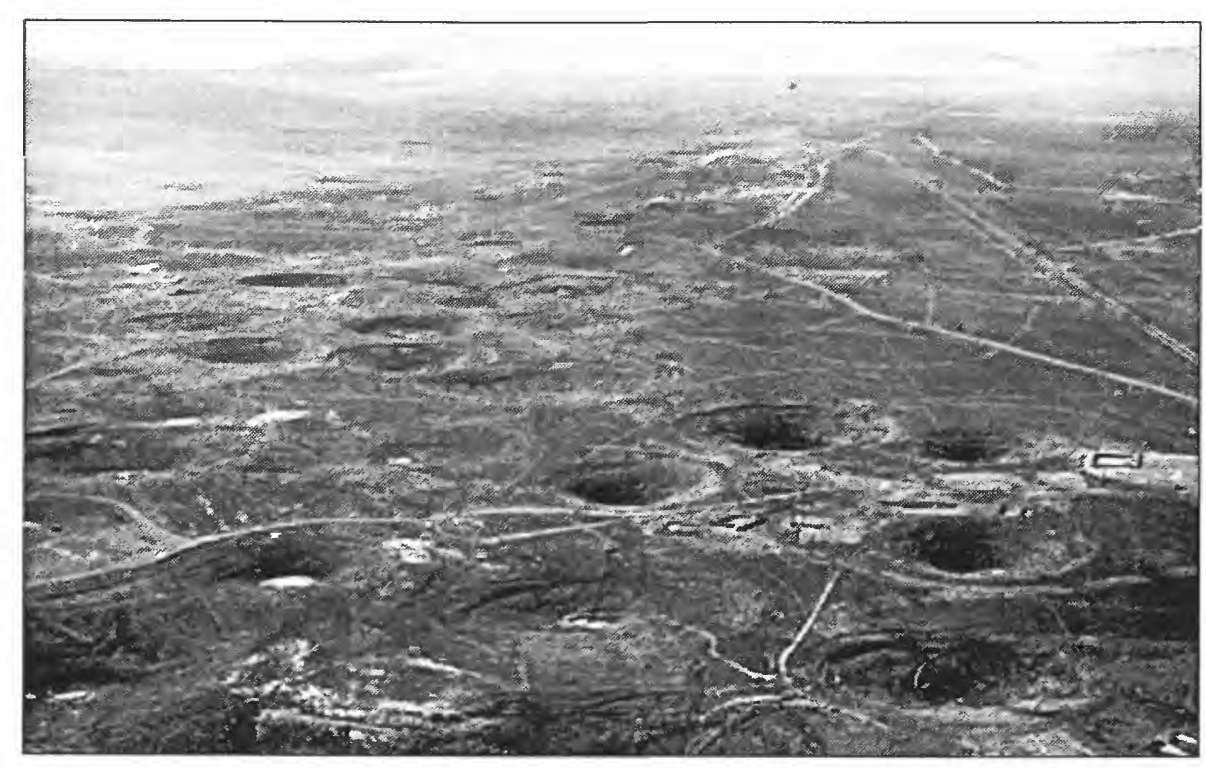

Figure 3. Surface-disruption features. View southeast, toward Yucca Lake. (Photograph courtesy of U.S. Department of Energy.) 

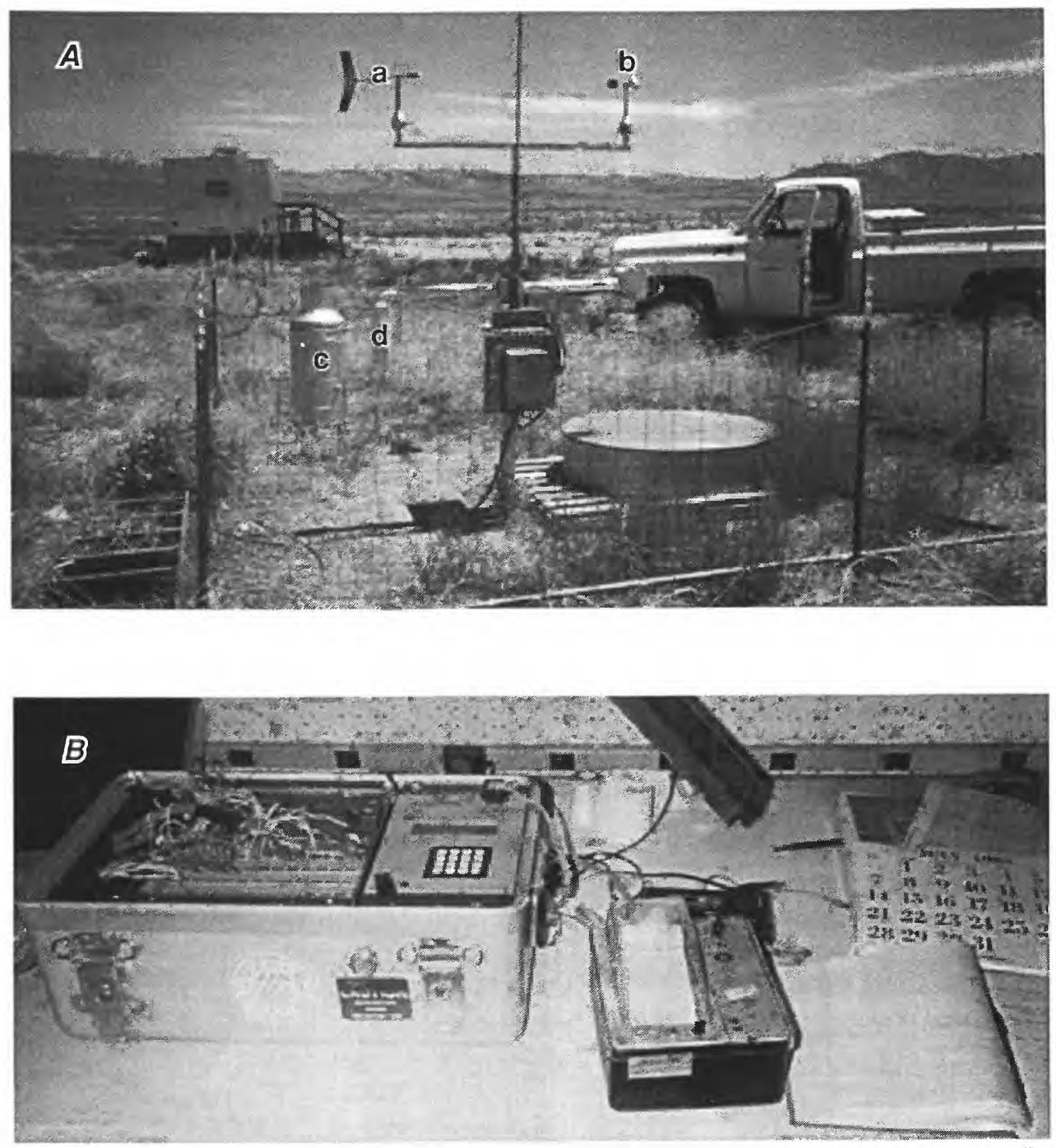

Figure 4. Weather-station instruments. A, Measuring devices: wind vane (a), cup anemometer (b), rain gage (c), relative-humidity probe with temperature sensor (d). Instrument trailer in left background. $B$, CR 7 data logger with printer attached. 

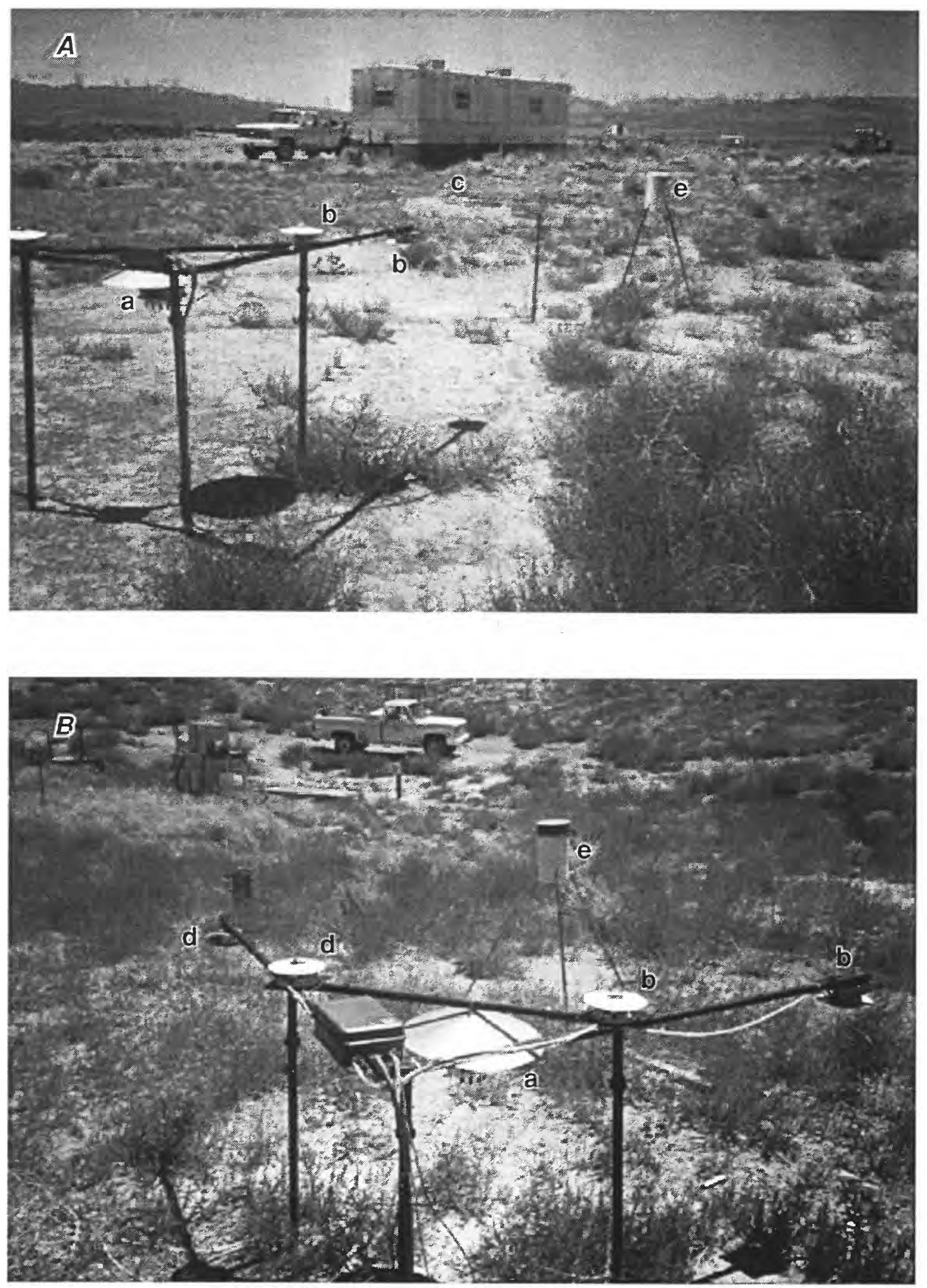

Figure 5. Instruments for collecting bare-soil evaporation data: thermistor psychrometers (a), Eppley pyranometers (b), net radiometer (c), pyrgeometers (d), I-R gun shelter (e). A, Station at original land surface. Instrument trailer in background. $B$, Station in crater 1 . 
Table 1. Radiation and temperature field data for undisturbed-land-surface site, May 1985 through June 1986

[Daily mean radiation: Pyranometer measured short-wave radiation; pyrgeometer measured long-wave radiation; net radiometer measured net radiation. In, incoming radiation; Out, outgoing radiation. --, no data or not calculated]

\begin{tabular}{|c|c|c|c|c|c|c|c|c|c|}
\hline \multirow{3}{*}{ Date } & \multicolumn{5}{|c|}{$\begin{array}{c}\text { Daily mean radiation } \\
\text { (watts per square meter) }\end{array}$} & \multicolumn{4}{|c|}{$\begin{array}{c}\text { Temperature } \\
\text { (degrees Celsius) }\end{array}$} \\
\hline & \multicolumn{2}{|c|}{ Long wave } & \multicolumn{2}{|c|}{ Short wave } & \multirow{2}{*}{ Net } & \multirow{2}{*}{$\begin{array}{c}\text { Daily mean } \\
\text { alr } \\
\text { temperature }\end{array}$} & \multicolumn{2}{|c|}{ Soil surface t } & temperature \\
\hline & In & out & In & Out & & & Mean & Maximum & Minimum \\
\hline \multicolumn{10}{|c|}{ May 1985} \\
\hline $\begin{array}{l}1 \ldots \ldots \\
2 \ldots \ldots \\
3 \ldots \ldots \\
4 \ldots \ldots \\
5 \ldots \ldots\end{array}$ & $\begin{array}{l}275 \\
295 \\
285 \\
263 \\
278\end{array}$ & $\begin{array}{l}410 \\
405 \\
407 \\
400 \\
394\end{array}$ & $\begin{array}{l}324 \\
277 \\
332 \\
331 \\
284\end{array}$ & $\begin{array}{r}102 \\
88 \\
109 \\
108 \\
92\end{array}$ & $\begin{array}{l}69 \\
55 \\
77 \\
68 \\
55\end{array}$ & $\begin{array}{l}18.8 \\
20.0 \\
21.3 \\
17.5 \\
16.6\end{array}$ & $\begin{array}{l}21.0 \\
21.4 \\
22.2 \\
19.9 \\
19.3\end{array}$ & $\begin{array}{l}43.7 \\
43.6 \\
39.6 \\
41.9 \\
39.9\end{array}$ & $\begin{array}{l}3.7 \\
6.6 \\
9.4 \\
3.8 \\
4.8\end{array}$ \\
\hline $\begin{array}{c}6 \ldots \ldots \ldots \\
7 \ldots \ldots \ldots \\
8 \ldots \ldots \\
9 \ldots \ldots \\
10 \ldots \ldots\end{array}$ & $\begin{array}{l}275 \\
257 \\
277 \\
300 \\
274\end{array}$ & $\begin{array}{l}-- \\
384 \\
379 \\
373 \\
347\end{array}$ & $\begin{array}{l}512 \\
343 \\
285 \\
250 \\
252\end{array}$ & $\begin{array}{r}168 \\
114 \\
93 \\
57 \\
58\end{array}$ & $\begin{array}{r}163 \\
81 \\
62 \\
76 \\
77\end{array}$ & $\begin{array}{l}21.6 \\
16.3 \\
18.5 \\
18.0 \\
12.9\end{array}$ & $\begin{array}{l}26.2 \\
18 \cdot 3 \\
18.8 \\
19.3 \\
14.4\end{array}$ & $\begin{array}{l}38.4 \\
38.0 \\
36.8 \\
36.2 \\
31.0\end{array}$ & $\begin{array}{r}9.0 \\
3.4 \\
6.5 \\
11.7 \\
5.5\end{array}$ \\
\hline $\begin{array}{l}11 \ldots \ldots \\
12 \ldots \ldots \\
13 \ldots \ldots \\
14 \ldots \ldots \ldots \\
15 \ldots \ldots\end{array}$ & $\begin{array}{r}262 \\
269 \\
-- \\
-- \\
--\end{array}$ & $\begin{array}{r}375 \\
383 \\
-- \\
-- \\
--\end{array}$ & $\begin{array}{r}333 \\
336 \\
-- \\
-- \\
--\end{array}$ & $\begin{array}{l}81 \\
83 \\
-- \\
-- \\
--\end{array}$ & $\begin{array}{r}103 \\
99 \\
-- \\
-- \\
--\end{array}$ & $\begin{array}{c}13.0 \\
16.1 \\
-- \\
-- \\
--\end{array}$ & $\begin{array}{c}17.1 \\
18.9 \\
-- \\
-- \\
--\end{array}$ & $\begin{array}{l}37.7 \\
41.6 \\
-- \\
-- \\
--\end{array}$ & $\begin{array}{l}3.7 \\
6.5 \\
-- \\
-- \\
--\end{array}$ \\
\hline $\begin{array}{l}16 \ldots \ldots \ldots \\
17 \ldots \ldots \ldots \\
18 \ldots \ldots \ldots \\
19 \ldots \ldots \ldots \\
20 \ldots \ldots \ldots\end{array}$ & $\begin{array}{l}-- \\
-- \\
-- \\
-- \\
--\end{array}$ & $\begin{array}{l}-- \\
-- \\
-- \\
-- \\
--\end{array}$ & $\begin{array}{l}-- \\
-- \\
-- \\
-- \\
--\end{array}$ & $\begin{array}{l}-- \\
-- \\
-- \\
-- \\
--\end{array}$ & $\begin{array}{l}-- \\
-- \\
-- \\
-- \\
--\end{array}$ & $\begin{array}{l}-- \\
-- \\
-- \\
-- \\
--\end{array}$ & $\begin{array}{l}-- \\
-- \\
-- \\
-- \\
--\end{array}$ & $\begin{array}{l}-- \\
-- \\
-- \\
-- \\
--\end{array}$ & $\begin{array}{l}-- \\
-- \\
-- \\
--\end{array}$ \\
\hline $\begin{array}{l}21 \ldots \ldots \ldots \\
22 \ldots \ldots \ldots \\
23 \ldots \ldots \ldots \\
24 \ldots \ldots \ldots \\
25 \ldots \ldots \ldots\end{array}$ & $\begin{array}{l}278 \\
285 \\
285 \\
295 \\
281\end{array}$ & $\begin{array}{l}.419 \\
416 \\
422 \\
420 \\
403\end{array}$ & $\begin{array}{l}399 \\
323 \\
342 \\
336 \\
306\end{array}$ & $\begin{array}{r}100 \\
79 \\
87 \\
85 \\
79\end{array}$ & $\begin{array}{r}115 \\
81 \\
91 \\
94 \\
77\end{array}$ & $\begin{array}{l}20.2 \\
19.7 \\
20.8 \\
22.1 \\
20.1\end{array}$ & $\begin{array}{l}24.9 \\
23.8 \\
24.9 \\
25.3 \\
22.4\end{array}$ & $\begin{array}{l}47.8 \\
50.4 \\
48.2 \\
47.0 \\
47.4\end{array}$ & $\begin{array}{l}6.9 \\
6.6 \\
6.8 \\
9.4 \\
7.7\end{array}$ \\
\hline $\begin{array}{l}26 \ldots \ldots \ldots \\
27 \ldots \ldots \ldots \\
28 \ldots \ldots \ldots \\
29 \ldots \ldots \ldots \\
30 \ldots \ldots \ldots\end{array}$ & $\begin{array}{l}261 \\
260 \\
259 \\
255 \\
262\end{array}$ & $\begin{array}{l}396 \\
393 \\
396 \\
396 \\
389\end{array}$ & $\begin{array}{l}348 \\
328 \\
345 \\
359 \\
286\end{array}$ & $\begin{array}{l}90 \\
85 \\
90 \\
94 \\
73\end{array}$ & $\begin{array}{l}96 \\
84 \\
90 \\
99 \\
60\end{array}$ & $\begin{array}{l}18.4 \\
19.1 \\
19.9 \\
19.2 \\
16.8\end{array}$ & $\begin{array}{l}20.9 \\
20.7 \\
21.4 \\
21.5 \\
19.6\end{array}$ & $\begin{array}{l}41.6 \\
41.0 \\
42.5 \\
42.3 \\
46.4\end{array}$ & $\begin{array}{l}4.3 \\
5.1 \\
6.9 \\
6.7 \\
2.6\end{array}$ \\
\hline $31 \ldots \ldots \ldots$ & 267 & 391 & 350 & 92 & 105 & 17.3 & 21.1 & 40.9 & 5.2 \\
\hline Mean..... & $\overline{274}$ & $\overline{395}$ & $\overline{330}$ & 92 & $\overline{86}$ & $\overline{18.4}$ & $\overline{21.0}$ & $\overline{41.9}$ & $\overline{6.2}$ \\
\hline \multicolumn{10}{|c|}{ June 1985} \\
\hline $\begin{array}{l}1 \ldots \ldots \\
2 \ldots \ldots \\
3 \ldots \ldots \\
4 \ldots \ldots \\
5 \ldots \ldots\end{array}$ & $\begin{array}{l}255 \\
276 \\
310 \\
287 \\
304\end{array}$ & $\begin{array}{r}390 \\
376 \\
-- \\
405 \\
425\end{array}$ & $\begin{array}{l}356 \\
270 \\
277 \\
338 \\
353\end{array}$ & $\begin{array}{l}92 \\
68 \\
51 \\
76 \\
82\end{array}$ & $\begin{array}{r}99 \\
66 \\
106 \\
105 \\
107\end{array}$ & $\begin{array}{l}16.0 \\
15.3 \\
16.2 \\
20.7 \\
23.7\end{array}$ & $\begin{array}{l}20.8 \\
19.1 \\
16.8 \\
22.0 \\
25.9\end{array}$ & $\begin{array}{l}43.6 \\
38.3 \\
29.8 \\
45.3 \\
48.5\end{array}$ & $\begin{array}{l}4.9 \\
4.3 \\
8.7 \\
5.9 \\
9.4\end{array}$ \\
\hline $\begin{array}{c}6 \ldots \ldots \ldots \\
7 \ldots \ldots \ldots \\
8 \ldots \ldots \ldots \\
9 \ldots \ldots \ldots \\
10 \ldots \ldots\end{array}$ & $\begin{array}{l}305 \\
302 \\
300 \\
297 \\
294\end{array}$ & $\begin{array}{l}434 \\
433 \\
439 \\
444 \\
415\end{array}$ & $\begin{array}{l}351 \\
345 \\
350 \\
352 \\
267\end{array}$ & $\begin{array}{l}83 \\
85 \\
86 \\
86 \\
62\end{array}$ & $\begin{array}{c}102 \\
-- \\
-- \\
-- \\
--\end{array}$ & $\begin{array}{l}24.7 \\
25.6 \\
25.8 \\
25.8 \\
24.5\end{array}$ & $\begin{array}{l}27.4 \\
27.7 \\
28.3 \\
28.6 \\
24.0\end{array}$ & $\begin{array}{l}48.9 \\
48.9 \\
51.6 \\
52.6 \\
52.8\end{array}$ & $\begin{array}{r}10.4 \\
10.4 \\
10.9 \\
9.4 \\
12.6\end{array}$ \\
\hline $\begin{array}{l}11 \ldots \ldots \\
12 \ldots \ldots \\
13 \ldots \ldots \\
14 \ldots \ldots \\
15 \ldots \ldots \ldots\end{array}$ & $\begin{array}{l}302 \\
305 \\
308 \\
306 \\
304\end{array}$ & $\begin{array}{l}458 \\
452 \\
453 \\
457 \\
457\end{array}$ & $\begin{array}{l}348 \\
350 \\
347 \\
347 \\
350\end{array}$ & $\begin{array}{l}82 \\
83 \\
83 \\
82 \\
84\end{array}$ & $\begin{array}{l}84 \\
91 \\
93 \\
90 \\
92\end{array}$ & $\begin{array}{l}28.3 \\
26.5 \\
26.9 \\
26.9 \\
26.9\end{array}$ & $\begin{array}{l}30.3 \\
29.7 \\
30.0 \\
30.4 \\
29.5\end{array}$ & $\begin{array}{l}54.7 \\
55.0 \\
54.4 \\
55.0 \\
45.4\end{array}$ & $\begin{array}{l}14.1 \\
10.8 \\
12.9 \\
12.0 \\
11.8\end{array}$ \\
\hline
\end{tabular}


Table 1. Radiation and temperature field data for undisturbed-land-surface site, May 1985 through June 1986 -Continued

\begin{tabular}{|c|c|c|c|c|c|c|c|c|c|}
\hline \multirow{3}{*}{ Date } & \multicolumn{5}{|c|}{$\begin{array}{l}\text { Daily mean radiation } \\
\text { (watts per square meter) }\end{array}$} & \multicolumn{4}{|c|}{$\begin{array}{c}\text { Temperature } \\
\text { (degrees Celsius) }\end{array}$} \\
\hline & \multicolumn{2}{|c|}{ Long wave } & \multicolumn{2}{|c|}{ Short wave } & \multirow{2}{*}{ Net } & \multirow{2}{*}{$\begin{array}{c}\text { Daily mean } \\
\text { air } \\
\text { temperature }\end{array}$} & \multirow{2}{*}{$\frac{\text { Soil }}{\text { Mean }}$} & \multirow{2}{*}{$\frac{\text { surface } t}{\text { Maximum }}$} & temperature \\
\hline & In & out & In & Out & & & & & Minimum \\
\hline \multicolumn{10}{|c|}{ June 1985 -Continued } \\
\hline $\begin{array}{l}16 \ldots \ldots \ldots \\
17 \ldots \ldots \ldots \\
18 \ldots \ldots \ldots \\
19 \ldots \ldots \ldots \\
20 \ldots \ldots \ldots\end{array}$ & $\begin{array}{l}304 \\
305 \\
323 \\
320 \\
295\end{array}$ & $\begin{array}{l}457 \\
462 \\
467 \\
465 \\
446\end{array}$ & $\begin{array}{l}356 \\
354 \\
318 \\
353 \\
357\end{array}$ & $\begin{array}{l}86 \\
84 \\
73 \\
85 \\
88\end{array}$ & $\begin{array}{l}-- \\
67 \\
72 \\
97 \\
95\end{array}$ & $\begin{array}{l}26.6 \\
27.5 \\
29.0 \\
28.6 \\
25.5\end{array}$ & $\begin{array}{l}30.1 \\
31.1 \\
32.4 \\
31.6 \\
28.6\end{array}$ & $\begin{array}{l}46.5 \\
48.4 \\
51.3 \\
46.2 \\
41.9\end{array}$ & $\begin{array}{l}12.5 \\
12.1 \\
14.9 \\
14.3 \\
13.6\end{array}$ \\
\hline $\begin{array}{l}21 \ldots \ldots \ldots \\
22 \ldots \ldots \ldots \\
23 \ldots \ldots \ldots \\
24 \ldots \ldots \ldots \\
25 \ldots \ldots \ldots \ldots\end{array}$ & $\begin{array}{l}276 \\
285 \\
295 \\
314 \\
247\end{array}$ & $\begin{array}{l}444 \\
438 \\
426 \\
414 \\
413\end{array}$ & $\begin{array}{l}360 \\
357 \\
305 \\
232 \\
369\end{array}$ & $\begin{array}{l}88 \\
88 \\
74 \\
53 \\
92\end{array}$ & $\begin{array}{l}88 \\
97 \\
78 \\
56 \\
99\end{array}$ & $\begin{array}{l}24.0 \\
24.8 \\
24.1 \\
23.1 \\
20.9\end{array}$ & $\begin{array}{l}27.6 \\
27.6 \\
26.5 \\
25.2 \\
23.7\end{array}$ & $\begin{array}{l}45.3 \\
41.5 \\
40.4 \\
36.5 \\
35.8\end{array}$ & $\begin{array}{r}8.8 \\
12.0 \\
10.8 \\
17.0 \\
11.6\end{array}$ \\
\hline $\begin{array}{l}26 \ldots \ldots \ldots \\
27 \ldots \ldots \ldots \\
28 \ldots \ldots \ldots \ldots \\
29 \ldots \ldots \ldots \\
30 \ldots \ldots \ldots\end{array}$ & $\begin{array}{l}208 \\
238 \\
254 \\
277 \\
238\end{array}$ & $\begin{array}{l}426 \\
433 \\
422 \\
403 \\
422\end{array}$ & $\begin{array}{l}367 \\
365 \\
312 \\
207 \\
369\end{array}$ & $\begin{array}{l}89 \\
89 \\
76 \\
49 \\
91\end{array}$ & $\begin{array}{r}86 \\
94 \\
78 \\
35 \\
101\end{array}$ & $\begin{array}{l}20.8 \\
21.7 \\
22.8 \\
22.4 \\
20.5\end{array}$ & $\begin{array}{l}24.8 \\
25.3 \\
25.3 \\
24.3 \\
23.9\end{array}$ & $\begin{array}{l}40.4 \\
42.1 \\
41.2 \\
35.7 \\
40.0\end{array}$ & $\begin{array}{r}9.1 \\
5.6 \\
7.2 \\
13.4 \\
5.8\end{array}$ \\
\hline Mean..... & $\overline{288}$ & $\overline{434}$ & $\overline{333}$ & 80 & 87 & $\overline{23.9}$ & $\overline{26.6}$ & $\overline{45.3}$ & 10.6 \\
\hline \multicolumn{10}{|c|}{ July 1.985} \\
\hline $\begin{array}{l}1 \ldots \ldots \\
2 \ldots \ldots \\
3 \ldots \ldots \\
4 \ldots \ldots \\
5 \ldots \ldots\end{array}$ & $\begin{array}{l}242 \\
199 \\
181 \\
241 \\
251\end{array}$ & $\begin{array}{l}443 \\
454 \\
460 \\
467 \\
472\end{array}$ & $\begin{array}{l}357 \\
361 \\
356 \\
353 \\
335\end{array}$ & $\begin{array}{l}88 \\
89 \\
89 \\
89 \\
83\end{array}$ & $\begin{array}{l}95 \\
90 \\
92 \\
97 \\
86\end{array}$ & $\begin{array}{l}23.1 \\
25.5 \\
27.4 \\
28.6 \\
29.4\end{array}$ & $\begin{array}{l}26.7 \\
29.4 \\
30.7 \\
31.7 \\
32.7\end{array}$ & $\begin{array}{l}44.6 \\
47.8 \\
48.3 \\
47.4 \\
51.3\end{array}$ & $\begin{array}{l}7.6 \\
10.0 \\
10.7 \\
13.2 \\
15.0\end{array}$ \\
\hline $\begin{array}{c}6 \ldots \ldots \ldots \\
7 \ldots \ldots \ldots \\
8 \ldots \ldots \ldots \\
9 \ldots \ldots \ldots \\
10 \ldots \ldots \ldots\end{array}$ & $\begin{array}{l}282 \\
279 \\
278 \\
307 \\
298\end{array}$ & $\begin{array}{l}475 \\
456 \\
459 \\
485 \\
485\end{array}$ & $\begin{array}{l}291 \\
296 \\
294 \\
309 \\
305\end{array}$ & $\begin{array}{l}73 \\
70 \\
69 \\
74 \\
71\end{array}$ & $\begin{array}{r}76 \\
88 \\
81 \\
100 \\
96\end{array}$ & $\begin{array}{l}31.4 \\
28.2 \\
28.8 \\
31.3 \\
31.1\end{array}$ & $\begin{array}{l}34.2 \\
31.4 \\
32.0 \\
34.2 \\
34.4\end{array}$ & $\begin{array}{l}46.5 \\
46.6 \\
48.8 \\
46.8 \\
45.0\end{array}$ & $\begin{array}{l}21.8 \\
17.7 \\
15.8 \\
23.6 \\
24.1\end{array}$ \\
\hline $\begin{array}{l}11 \ldots \ldots \ldots \\
12 \ldots \ldots \ldots \\
13 \ldots \ldots \ldots \\
14 \ldots \ldots \ldots \\
15 \ldots \ldots \ldots\end{array}$ & $\begin{array}{r}274 \\
260 \\
-- \\
-- \\
--\end{array}$ & $\begin{array}{l}480 \\
477 \\
469 \\
457 \\
454\end{array}$ & $\begin{array}{l}331 \\
327 \\
341 \\
315 \\
299\end{array}$ & $\begin{array}{l}81 \\
78 \\
83 \\
78 \\
75\end{array}$ & $\begin{array}{r}101 \\
95 \\
98 \\
88 \\
80\end{array}$ & $\begin{array}{l}29.9 \\
29.7 \\
28.0 \\
27.1 \\
27.3\end{array}$ & $\begin{array}{l}33.2 \\
33.2 \\
31.3 \\
29.9 \\
30.0\end{array}$ & $\begin{array}{l}44.8 \\
45.8 \\
46.8 \\
44.6 \\
43.7\end{array}$ & $\begin{array}{l}18.9 \\
21.6 \\
14.5 \\
15.0 \\
14.3\end{array}$ \\
\hline $\begin{array}{l}16 \ldots \ldots \ldots \\
17 \ldots \ldots \ldots \\
18 \ldots \ldots \ldots \\
19 \ldots \ldots \ldots \\
20 \ldots \ldots \ldots\end{array}$ & $\begin{array}{l}-- \\
-- \\
-- \\
--\end{array}$ & $\begin{array}{l}439 \\
463 \\
416 \\
430 \\
427\end{array}$ & $\begin{array}{l}201 \\
314 \\
191 \\
238 \\
247\end{array}$ & $\begin{array}{l}47 \\
77 \\
37 \\
50 \\
45\end{array}$ & $\begin{array}{l}43 \\
99 \\
62 \\
78 \\
82\end{array}$ & $\begin{array}{l}25.9 \\
27.3 \\
23.0 \\
21.9 \\
21.7\end{array}$ & $\begin{array}{l}28.1 \\
30.3 \\
25.3 \\
25.0 \\
24.6\end{array}$ & $\begin{array}{l}39.9 \\
42.0 \\
36.4 \\
39.4 \\
38.6\end{array}$ & $\begin{array}{l}16.3 \\
16.4 \\
18.4 \\
16.9 \\
18.1\end{array}$ \\
\hline $\begin{array}{l}21 \ldots \ldots \ldots \\
22 \ldots \ldots \ldots \\
23 \ldots \ldots \ldots \\
24 \ldots \ldots \\
25 \ldots \ldots \ldots\end{array}$ & $\begin{array}{l}-- \\
-- \\
-- \\
--\end{array}$ & $\begin{array}{l}422 \\
442 \\
454 \\
467 \\
458\end{array}$ & $\begin{array}{l}274 \\
337 \\
315 \\
318 \\
298\end{array}$ & $\begin{array}{l}56 \\
78 \\
72 \\
73 \\
69\end{array}$ & $\begin{array}{r}75 \\
109 \\
97 \\
86 \\
86\end{array}$ & $\begin{array}{l}22.5 \\
24.1 \\
26.0 \\
27.7 \\
26.1\end{array}$ & $\begin{array}{l}25.4 \\
27.1 \\
29.2 \\
31.6 \\
29.2\end{array}$ & $\begin{array}{l}36.2 \\
40.1 \\
41.8 \\
46.3 \\
44.1\end{array}$ & $\begin{array}{l}17.3 \\
14.0 \\
14.8 \\
18.7 \\
17.4\end{array}$ \\
\hline $\begin{array}{l}26 \ldots \ldots \ldots \\
27 \ldots \ldots \ldots \\
28 \ldots \ldots \ldots \\
29 \ldots \ldots \\
30 \ldots \ldots \ldots\end{array}$ & $\begin{array}{l}-- \\
-- \\
-- \\
-- \\
--\end{array}$ & $\begin{array}{l}460 \\
460 \\
455 \\
439 \\
439\end{array}$ & $\begin{array}{l}302 \\
307 \\
331 \\
339 \\
341\end{array}$ & $\begin{array}{l}69 \\
71 \\
80 \\
83 \\
83\end{array}$ & $\begin{array}{r}84 \\
88 \\
100 \\
104 \\
93\end{array}$ & $\begin{array}{l}26.1 \\
26.7 \\
25.5 \\
24.2 \\
23.2\end{array}$ & $\begin{array}{l}29.6 \\
29.9 \\
28.6 \\
26.6 \\
26.5\end{array}$ & $\begin{array}{l}45.1 \\
43.9 \\
41.7 \\
38.1 \\
38.1\end{array}$ & $\begin{array}{l}15.8 \\
15.4 \\
16.6 \\
13.7 \\
15.7\end{array}$ \\
\hline $31 \ldots \ldots \ldots$ & -- & 427 & 345 & 86 & 102 & 22.0 & 24.4 & 37.9 & 7.6 \\
\hline Mean..... & $\overline{258}$ & $\overline{455}$ & $\overline{309}$ & 73 & 89 & $\overline{26.5}$ & $\overline{29.6}$ & $\overline{43.5}$ & 16.0 \\
\hline
\end{tabular}


Table 1. Radiation and temperature field data for undisturbed-land-surface site, May 1985 through June 1986-Continued

\begin{tabular}{|c|c|c|c|c|c|c|c|c|c|}
\hline \multirow{3}{*}{ Date } & \multicolumn{5}{|c|}{$\begin{array}{l}\text { Daily mean radiation, } \\
\text { (watts per square meter) }\end{array}$} & \multicolumn{4}{|c|}{$\begin{array}{c}\text { Temperature } \\
\text { (degrees Celsius) }\end{array}$} \\
\hline & \multicolumn{2}{|c|}{ Long wave } & \multicolumn{2}{|c|}{ Short wave } & \multirow{2}{*}{ Net } & \multirow{2}{*}{$\begin{array}{c}\text { Daily mean } \\
\text { air } \\
\text { temperature }\end{array}$} & \multicolumn{2}{|c|}{ Soil surface } & temperature \\
\hline & In & Out & In & Out & & & Mean & Maximum & Minimum \\
\hline \multicolumn{10}{|c|}{ August 1985} \\
\hline $\begin{array}{l}1 \ldots \ldots \\
2 \ldots \ldots \\
3 \ldots \ldots \\
4 \ldots \ldots \\
5 \ldots \ldots\end{array}$ & $\begin{array}{l}-- \\
-- \\
-- \\
-- \\
--\end{array}$ & $\begin{array}{l}428 \\
436 \\
443 \\
442 \\
442\end{array}$ & $\begin{array}{l}344 \\
340 \\
337 \\
338 \\
338\end{array}$ & $\begin{array}{l}87 \\
84 \\
82 \\
83 \\
83\end{array}$ & $\begin{array}{r}103 \\
93 \\
89 \\
92 \\
87\end{array}$ & $\begin{array}{l}22.8 \\
22.1 \\
22.4 \\
23.4 \\
23.1\end{array}$ & $\begin{array}{l}25.0 \\
25.6 \\
25.9 \\
26.3 \\
26.3\end{array}$ & $\begin{array}{l}36.2 \\
39.3 \\
43.4 \\
41.6 \\
44.1\end{array}$ & $\begin{array}{r}10.0 \\
9.9 \\
8.7 \\
9.3 \\
8.5\end{array}$ \\
\hline $\begin{array}{c}6 \ldots \ldots \ldots \\
7 \ldots \ldots \ldots \\
8 \ldots \ldots \ldots \\
9 \ldots \ldots \ldots \\
10 \ldots \ldots\end{array}$ & $\begin{array}{l}-- \\
-- \\
-- \\
-- \\
--\end{array}$ & $\begin{array}{l}442 \\
435 \\
436 \\
450 \\
439\end{array}$ & $\begin{array}{l}336 \\
317 \\
319 \\
329 \\
327\end{array}$ & $\begin{array}{l}84 \\
79 \\
81 \\
81 \\
82\end{array}$ & $\begin{array}{l}89 \\
83 \\
89 \\
80 \\
93\end{array}$ & $\begin{array}{l}23.1 \\
23.2 \\
24.1 \\
24.8 \\
24.1\end{array}$ & $\begin{array}{l}26.0 \\
25.5 \\
26.2 \\
27.8 \\
26.2\end{array}$ & $\begin{array}{l}44.6 \\
40.5 \\
39.3 \\
45.5 \\
40.6\end{array}$ & $\begin{array}{r}8.3 \\
9.3 \\
12.0 \\
13.7 \\
9.6\end{array}$ \\
\hline $\begin{array}{l}11 \ldots \ldots \ldots \\
12 \ldots \ldots \\
13 \ldots \ldots \\
14 \ldots \ldots \ldots \\
15 \ldots \ldots \ldots\end{array}$ & $\begin{array}{l}-- \\
-- \\
-- \\
-- \\
--\end{array}$ & $\begin{array}{l}447 \\
443 \\
435 \\
435 \\
442\end{array}$ & $\begin{array}{l}317 \\
320 \\
322 \\
291 \\
314\end{array}$ & $\begin{array}{l}78 \\
80 \\
81 \\
71 \\
78\end{array}$ & $\begin{array}{l}75 \\
79 \\
85 \\
68 \\
83\end{array}$ & $\begin{array}{l}24.5 \\
24.3 \\
22.1 \\
22.4 \\
23.2\end{array}$ & $\begin{array}{l}27.5 \\
27.3 \\
24.9 \\
25.2 \\
26.0\end{array}$ & $\begin{array}{l}44.0 \\
40.4 \\
41.4 \\
43.8 \\
43.5\end{array}$ & $\begin{array}{r}11.4 \\
16.0 \\
8.4 \\
8.3 \\
10.2\end{array}$ \\
\hline $\begin{array}{l}16 \ldots \ldots \ldots \\
17 \ldots \ldots \ldots \\
18 \ldots \ldots \\
19 \ldots \ldots \ldots \\
20 \ldots \ldots \ldots\end{array}$ & $\begin{array}{l}-- \\
-- \\
-- \\
-- \\
--\end{array}$ & $\begin{array}{l}440 \\
431 \\
422 \\
416 \\
429\end{array}$ & $\begin{array}{l}301 \\
281 \\
323 \\
265 \\
324\end{array}$ & $\begin{array}{l}74 \\
70 \\
83 \\
67 \\
82\end{array}$ & $\begin{array}{l}83 \\
77 \\
90 \\
67 \\
89\end{array}$ & $\begin{array}{l}23.6 \\
23.4 \\
21.4 \\
21.0 \\
23.1\end{array}$ & $\begin{array}{l}26.0 \\
25.4 \\
23.5 \\
23.4 \\
26.0\end{array}$ & $\begin{array}{l}41.6 \\
38.6 \\
37.5 \\
39.1 \\
39.8\end{array}$ & $\begin{array}{r}11.5 \\
11.9 \\
9.5 \\
6.6 \\
11.1\end{array}$ \\
\hline $\begin{array}{l}21 \ldots \ldots \ldots \\
22 \ldots \ldots \ldots \\
23 \ldots \ldots \\
24 \ldots \ldots \ldots \\
25 \ldots \ldots \ldots\end{array}$ & $\begin{array}{l}-- \\
-- \\
-- \\
--\end{array}$ & $\begin{array}{l}421 \\
429 \\
446 \\
453 \\
453\end{array}$ & $\begin{array}{l}284 \\
295 \\
303 \\
301 \\
293\end{array}$ & $\begin{array}{l}71 \\
73 \\
73 \\
73 \\
73\end{array}$ & $\begin{array}{l}69 \\
81 \\
75 \\
73 \\
79\end{array}$ & $\begin{array}{l}22.1 \\
21.5 \\
24.2 \\
25.9 \\
26.5\end{array}$ & $\begin{array}{l}24.7 \\
25.0 \\
28.3 \\
29.7 \\
29.6\end{array}$ & $\begin{array}{l}38.4 \\
43.0 \\
49.4 \\
50.5 \\
47.5\end{array}$ & $\begin{array}{r}11.7 \\
8.1 \\
9.0 \\
10.7 \\
12.8\end{array}$ \\
\hline $\begin{array}{l}26 \ldots \ldots \ldots \\
27 \ldots \ldots \ldots \\
28 \ldots \ldots \ldots \\
29 \ldots \ldots \ldots \\
30 \ldots \ldots \ldots\end{array}$ & $\begin{array}{l}-- \\
-- \\
-- \\
-- \\
--\end{array}$ & $\begin{array}{l}455 \\
445 \\
431 \\
429 \\
426\end{array}$ & $\begin{array}{l}287 \\
298 \\
303 \\
296 \\
286\end{array}$ & $\begin{array}{l}71 \\
74 \\
76 \\
73 \\
70\end{array}$ & $\begin{array}{l}81 \\
82 \\
79 \\
74 \\
78\end{array}$ & $\begin{array}{l}27.3 \\
25.3 \\
23.4 \\
23.1 \\
24.3\end{array}$ & $\begin{array}{l}30.2 \\
2.8 \cdot 4 \\
26.3 \\
26.5 \\
27.1\end{array}$ & $\begin{array}{l}44.2 \\
43.1 \\
43.1 \\
44.8 \\
43.3\end{array}$ & $\begin{array}{r}16.6 \\
15.1 \\
10.7 \\
9.3 \\
10.7\end{array}$ \\
\hline $31 \ldots \ldots$ & -- & 400 & 292 & 72 & 78 & 25.0 & 28.0 & 43.5 & 13.5 \\
\hline Mean..... & -- & $\overline{436}$ & $\overline{310}$ & 77 & 82 & $\overline{23.6}$ & $\overline{26.5}$ & $\overline{42.5}$ & $\overline{10.7}$ \\
\hline \multicolumn{10}{|c|}{ Septamber 1985} \\
\hline $\begin{array}{l}1 \ldots \ldots \\
2 \ldots \ldots \\
3 \ldots \ldots \\
4 \ldots \ldots \\
5 \ldots \ldots\end{array}$ & $\begin{array}{l}-- \\
-- \\
-- \\
-- \\
--\end{array}$ & $\begin{array}{l}-- \\
-- \\
-- \\
-- \\
--\end{array}$ & $\begin{array}{l}286 \\
291 \\
293 \\
229 \\
200\end{array}$ & $\begin{array}{l}72 \\
73 \\
74 \\
54 \\
46\end{array}$ & $\begin{array}{l}24 \\
25 \\
20 \\
16 \\
16\end{array}$ & $\begin{array}{l}81.4 \\
76.8 \\
80.8 \\
54.5 \\
41.7\end{array}$ & $\begin{array}{l}26.8 \\
27.2 \\
23.5 \\
18.7 \\
18.9\end{array}$ & $\begin{array}{l}39.6 \\
38.0 \\
36.8 \\
32.2 \\
34.4\end{array}$ & $\begin{array}{r}13.5 \\
17.4 \\
11.4 \\
6.6 \\
9.1\end{array}$ \\
\hline $\begin{array}{c}6 \ldots \ldots \ldots \\
7 \ldots \ldots \ldots \\
8 \ldots \ldots \ldots \\
9 \ldots \ldots \ldots \\
10 \ldots \ldots \ldots\end{array}$ & $\begin{array}{l}-- \\
-- \\
-- \\
-- \\
--\end{array}$ & $\begin{array}{l}-- \\
-- \\
-- \\
-- \\
--\end{array}$ & $\begin{array}{l}196 \\
262 \\
243 \\
282 \\
277\end{array}$ & $\begin{array}{l}45 \\
63 \\
59 \\
71 \\
70\end{array}$ & $\begin{array}{l}14 \\
16 \\
18 \\
18 \\
15\end{array}$ & $\begin{array}{l}41.9 \\
76.5 \\
69.8 \\
77.3 \\
77.0\end{array}$ & $\begin{array}{l}16.4 \\
18.5 \\
20.2 \\
20.2 \\
17.4\end{array}$ & $\begin{array}{l}32.2 \\
32.2 \\
32.8 \\
28.9 \\
30.1\end{array}$ & $\begin{array}{r}5.4 \\
3.9 \\
8.6 \\
12.7 \\
4.5\end{array}$ \\
\hline $\begin{array}{l}11 \ldots \ldots \ldots \ldots \\
12 \ldots \ldots \ldots \\
13 \ldots \ldots \ldots \ldots \\
14 \ldots \ldots \ldots \ldots \\
15 \ldots \ldots \ldots \ldots\end{array}$ & $\begin{array}{l}-- \\
-- \\
-- \\
-- \\
--\end{array}$ & $\begin{array}{l}-- \\
-- \\
-- \\
-- \\
--\end{array}$ & $\begin{array}{l}205 \\
273 \\
263 \\
264 \\
260\end{array}$ & $\begin{array}{l}50 \\
67 \\
66 \\
67 \\
66\end{array}$ & $\begin{array}{l}12 \\
13 \\
16 \\
17 \\
18\end{array}$ & $\begin{array}{l}44.0 \\
65.1 \\
67.4 \\
67.6 \\
68.5\end{array}$ & $\begin{array}{l}13.8 \\
16.8 \\
18.6 \\
20.0 \\
20.3\end{array}$ & $\begin{array}{l}25.0 \\
36.9 \\
36.5 \\
36.4 \\
35.7\end{array}$ & $\begin{array}{r}4.5 \\
.2 \\
2.9 \\
3.9 \\
6.9\end{array}$ \\
\hline
\end{tabular}


Table 1. Radiation and temperature field data for undisturbed-land-surface site, May 1985 through June 1986 -Continued

\begin{tabular}{|c|c|c|c|c|c|c|c|c|c|}
\hline \multirow{3}{*}{ Date } & \multicolumn{5}{|c|}{$\begin{array}{l}\text { Daily mean radiation } \\
\text { (watts per square meter) }\end{array}$} & \multicolumn{4}{|c|}{$\begin{array}{c}\text { Temperature } \\
\text { (degrees Celsius) }\end{array}$} \\
\hline & Long $w$ & wave & \multicolumn{2}{|c|}{ Short wave } & \multirow{2}{*}{ Net } & \multirow{2}{*}{$\begin{array}{c}\text { Daily mean } \\
\text { air } \\
\text { temperature }\end{array}$} & \multirow{2}{*}{$\begin{array}{l}\text { Soil } \\
\text { Mean }\end{array}$} & \multirow{2}{*}{$\frac{\text { surface } t}{\text { Maximum }}$} & temperature \\
\hline & In & $\overline{\text { out }}$ & In & $\overline{\text { out }}$ & & & & & Minimum \\
\hline \multicolumn{10}{|c|}{ September 1985-Continued } \\
\hline $\begin{array}{l}16 \ldots \ldots \ldots \\
17 \ldots \ldots \ldots \\
18 \ldots \ldots \ldots \\
19 \ldots \ldots \ldots \\
20 \ldots \ldots \ldots\end{array}$ & $\begin{array}{l}-- \\
-- \\
-- \\
--\end{array}$ & $\begin{array}{l}-- \\
-- \\
-- \\
-- \\
--\end{array}$ & $\begin{array}{r}266 \\
263 \\
70 \\
182 \\
251\end{array}$ & $\begin{array}{l}67 \\
67 \\
12 \\
37 \\
59\end{array}$ & $\begin{array}{l}17 \\
17 \\
15 \\
14 \\
14\end{array}$ & $\begin{array}{l}67.6 \\
72.9 \\
-8.3 \\
52.3 \\
70.5\end{array}$ & $\begin{array}{l}19.4 \\
18.8 \\
16.4 \\
16.6 \\
17.6\end{array}$ & $\begin{array}{l}34.4 \\
30.9 \\
22.1 \\
27.7 \\
36.3\end{array}$ & $\begin{array}{r}5.3 \\
5.2 \\
11.8 \\
8.1 \\
4.5\end{array}$ \\
\hline $\begin{array}{l}21 \ldots \ldots \ldots \\
22 \ldots \ldots \ldots \\
23 \ldots \ldots \ldots \\
24 \ldots \ldots \ldots \\
25 \ldots \ldots \ldots \ldots\end{array}$ & $\begin{array}{r}-- \\
-- \\
-- \\
290 \\
287\end{array}$ & $\begin{array}{l}-- \\
-- \\
-- \\
-- \\
--\end{array}$ & $\begin{array}{l}253 \\
252 \\
244 \\
246 \\
244\end{array}$ & $\begin{array}{l}61 \\
62 \\
58 \\
60 \\
59\end{array}$ & $\begin{array}{l}15 \\
16 \\
16 \\
18 \\
19\end{array}$ & $\begin{array}{l}66.9 \\
63.0 \\
56.6 \\
59.7 \\
51.4\end{array}$ & $\begin{array}{l}17.1 \\
18.9 \\
19.7 \\
21.4 \\
22.5\end{array}$ & $\begin{array}{l}34.8 \\
36.3 \\
41.3 \\
41.4 \\
42.8\end{array}$ & $\begin{array}{l}1.0 \\
4.3 \\
3.0 \\
5.0 \\
5.9\end{array}$ \\
\hline $\begin{array}{l}26 \ldots \ldots \ldots \\
27 \ldots \ldots \ldots \\
28 \ldots \ldots \ldots \\
29 \ldots \ldots \ldots \\
30 \ldots \ldots\end{array}$ & $\begin{array}{l}283 \\
313 \\
282 \\
250 \\
275\end{array}$ & $\begin{array}{l}-- \\
-- \\
-- \\
--\end{array}$ & $\begin{array}{l}240 \\
162 \\
230 \\
230 \\
223\end{array}$ & $\begin{array}{l}57 \\
37 \\
53 \\
54 \\
53\end{array}$ & $\begin{array}{l}18 \\
18 \\
18 \\
14 \\
15\end{array}$ & $\begin{array}{l}56.5 \\
33.7 \\
55.0 \\
46.2 \\
55.2\end{array}$ & $\begin{array}{l}20.7 \\
19.7 \\
21.2 \\
17.4 \\
18.0\end{array}$ & $\begin{array}{l}38.4 \\
33.0 \\
39.0 \\
35.5 \\
32.8\end{array}$ & $\begin{array}{l}5.0 \\
9.0 \\
7.4 \\
1.4 \\
4.5\end{array}$ \\
\hline Mean..... & $\overline{283}$ & -- & $\overline{239}$ & $\overline{58}$ & 17 & $\overline{59.7}$ & $\overline{19.4}$ & $\overline{34.5}$ & 6.4 \\
\hline \multicolumn{10}{|c|}{ October 1985} \\
\hline $\begin{array}{l}1 \ldots \ldots \\
2 \ldots \ldots \\
3 \ldots \ldots \\
4 \ldots \ldots \\
5 \ldots \ldots\end{array}$ & $\begin{array}{l}282 \\
262 \\
275 \\
281 \\
270\end{array}$ & $\begin{array}{l}-- \\
-- \\
-- \\
--\end{array}$ & $\begin{array}{l}208 \\
227 \\
227 \\
219 \\
225\end{array}$ & $\begin{array}{l}49 \\
56 \\
55 \\
54 \\
55\end{array}$ & $\begin{array}{l}43 \\
45 \\
47 \\
40 \\
50\end{array}$ & $\begin{array}{l}16.8 \\
15.9 \\
18.6 \\
20.0 \\
17.0\end{array}$ & $\begin{array}{l}20.2 \\
18.7 \\
21.5 \\
23.2 \\
19.7\end{array}$ & $\begin{array}{l}39.7 \\
38.2 \\
41.0 \\
40.6 \\
38.1\end{array}$ & $\begin{array}{l}6.2 \\
2.5 \\
5.8 \\
9.2 \\
3.6\end{array}$ \\
\hline $\begin{array}{c}6 \ldots \ldots \ldots \\
7 \ldots \ldots \\
8 \ldots \ldots \\
9 \ldots \ldots \\
10 \ldots \ldots\end{array}$ & $\begin{array}{l}301 \\
287 \\
286 \\
278 \\
270\end{array}$ & $\begin{array}{l}-- \\
-- \\
-- \\
--\end{array}$ & $\begin{array}{r}211 \\
210 \\
77 \\
107 \\
130\end{array}$ & $\begin{array}{l}53 \\
54 \\
11 \\
20 \\
27\end{array}$ & $\begin{array}{r}57 \\
55 \\
4 \\
29 \\
32\end{array}$ & $\begin{array}{r}18.4 \\
14.9 \\
9.5 \\
7.7 \\
10.7\end{array}$ & $\begin{array}{r}20.2 \\
17.2 \\
11.4 \\
9.4 \\
12.6\end{array}$ & $\begin{array}{l}33.6 \\
28.0 \\
18.5 \\
21.2 \\
23.6\end{array}$ & $\begin{array}{r}10.2 \\
5.5 \\
4.8 \\
3.1 \\
4.6\end{array}$ \\
\hline $\begin{array}{l}11 \ldots \ldots \ldots \\
12 \ldots \ldots \\
13 \ldots \ldots \\
14 \ldots \ldots \\
15 \ldots \ldots \ldots\end{array}$ & $\begin{array}{l}239 \\
246 \\
239 \\
214 \\
221\end{array}$ & $\begin{array}{l}-- \\
-- \\
-- \\
--\end{array}$ & $\begin{array}{l}213 \\
187 \\
209 \\
214 \\
211\end{array}$ & $\begin{array}{l}48 \\
42 \\
50 \\
52 \\
51\end{array}$ & $\begin{array}{l}57 \\
32 \\
45 \\
33 \\
38\end{array}$ & $\begin{array}{r}9.2 \\
9.9 \\
12.1 \\
11.2 \\
11.7\end{array}$ & $\begin{array}{l}11.5 \\
12.3 \\
14.4 \\
13.2 \\
14.2\end{array}$ & $\begin{array}{l}26.3 \\
26.8 \\
26.6 \\
27.3 \\
30.3\end{array}$ & $\begin{array}{r}-1.0 \\
-.8 \\
6.8 \\
3.3 \\
1.0\end{array}$ \\
\hline $\begin{array}{l}16 \ldots \ldots \ldots \\
17 \ldots \ldots \\
18 \ldots \ldots \\
19 \ldots \ldots \\
20 \ldots \ldots\end{array}$ & $\begin{array}{l}228 \\
236 \\
239 \\
244 \\
263\end{array}$ & $\begin{array}{l}-- \\
-- \\
-- \\
--\end{array}$ & $\begin{array}{l}205 \\
200 \\
198 \\
193 \\
153\end{array}$ & $\begin{array}{l}48 \\
46 \\
46 \\
45 \\
35\end{array}$ & $\begin{array}{l}36 \\
35 \\
39 \\
40 \\
25\end{array}$ & $\begin{array}{l}9.9 \\
11.4 \\
11.1 \\
10.8 \\
12.3\end{array}$ & $\begin{array}{l}12.8 \\
14.1 \\
13.6 \\
13.3 \\
14.0\end{array}$ & $\begin{array}{l}34.8 \\
35.1 \\
31.6 \\
30.3 \\
27.9\end{array}$ & $\begin{array}{r}-2.1 \\
-2.1 \\
-1.2 \\
-.6 \\
1.7\end{array}$ \\
\hline $\begin{array}{l}21 \ldots \ldots \\
22 \ldots \ldots \ldots \\
23 \ldots \ldots \ldots \\
24 \ldots \ldots \ldots \\
25 \ldots \ldots \ldots\end{array}$ & $\begin{array}{r}-- \\
2 \overline{39} \\
252 \\
250\end{array}$ & $\begin{array}{l}-- \\
-- \\
-- \\
-- \\
--\end{array}$ & $\begin{array}{l}-- \\
186 \\
182 \\
182\end{array}$ & $\begin{array}{l}-- \\
-- \\
42 \\
41\end{array}$ & $\begin{array}{l}-- \\
-- \\
33 \\
22 \\
27\end{array}$ & $\begin{array}{r}-- \\
\overline{8.4} \\
11.9 \\
11.8\end{array}$ & $\begin{array}{l}-- \\
11.0 \\
14.4 \\
14.2\end{array}$ & $\begin{array}{c}-- \\
\overline{--} \\
29.2 \\
33.1 \\
32.1\end{array}$ & $\begin{array}{r}-- \\
-- \\
-3.5 \\
.0 \\
.7\end{array}$ \\
\hline $\begin{array}{l}26 \ldots \ldots \ldots \\
27 \ldots \ldots \ldots \\
28 \ldots \ldots \ldots \\
29 \ldots \ldots \ldots \\
30 \ldots \ldots \ldots\end{array}$ & $\begin{array}{l}248 \\
247 \\
270 \\
244 \\
252\end{array}$ & $\begin{array}{l}-- \\
-- \\
-- \\
-- \\
--\end{array}$ & $\begin{array}{r}181 \\
178 \\
90 \\
174 \\
159\end{array}$ & $\begin{array}{l}41 \\
41 \\
18 \\
40 \\
37\end{array}$ & $\begin{array}{l}28 \\
28 \\
-6 \\
25 \\
23\end{array}$ & $\begin{array}{l}12.1 \\
11.7 \\
10.9 \\
10.8 \\
10.3\end{array}$ & $\begin{array}{l}14.6 \\
14.0 \\
12.7 \\
13.5 \\
12.5\end{array}$ & $\begin{array}{l}35.4 \\
34.4 \\
31.9 \\
32.6 \\
28.7\end{array}$ & $\begin{array}{r}.0 \\
-.3 \\
3.2 \\
.1 \\
-1.6\end{array}$ \\
\hline $31 \ldots \ldots \ldots$ & 245 & -- & 193 & 48 & 34 & 13.3 & 14.7 & 23.2 & 9.1 \\
\hline Mean..... & 255 & -- & $\overline{184}$ & 43 & $\overline{34}$ & $\overline{12.4}$ & $\overline{14.8}$ & $\overline{31.0}$ & 2.4 \\
\hline
\end{tabular}


Table 1. Radiation and temperature field data for undisturbed-land-surface site, May 1985 through June 1986 - Continued

\begin{tabular}{|c|c|c|c|c|c|c|c|c|c|}
\hline \multirow{3}{*}{ Date } & \multicolumn{5}{|c|}{$\begin{array}{l}\text { Daily mean radiation } \\
\text { (watts per square meter) }\end{array}$} & \multicolumn{4}{|c|}{$\begin{array}{c}\text { Temperature } \\
\text { (degrees Celsius) }\end{array}$} \\
\hline & \multicolumn{2}{|c|}{ Long wave } & \multicolumn{2}{|c|}{ Short wave } & \multirow{2}{*}{ Net } & \multirow{2}{*}{$\begin{array}{c}\text { Daily mean } \\
\text { air } \\
\text { temperature }\end{array}$} & \multirow{2}{*}{$\overline{\text { Soil }}$} & surface & temperature \\
\hline & In & out & In & $\overline{\text { Out }}$ & & & & Maximum & Minimum \\
\hline \multicolumn{10}{|c|}{ November 1985} \\
\hline $\begin{array}{l}1 \ldots \ldots \ldots \\
2 \ldots \ldots \\
3 \ldots \ldots \\
4 \ldots \ldots \ldots \\
5 \ldots \ldots \ldots\end{array}$ & $\begin{array}{l}- \\
-- \\
- \\
--\end{array}$ & $\begin{array}{l}-- \\
-- \\
-- \\
--\end{array}$ & $\begin{array}{l}-- \\
-- \\
-- \\
--\end{array}$ & $\begin{array}{l}-- \\
-- \\
-- \\
-- \\
--\end{array}$ & $\begin{array}{l}-- \\
-- \\
-- \\
-- \\
--\end{array}$ & $\begin{array}{l}-- \\
-- \\
-- \\
-- \\
--\end{array}$ & $\begin{array}{l}-- \\
-- \\
-- \\
--\end{array}$ & $\begin{array}{l}-- \\
-- \\
-- \\
--\end{array}$ & $\begin{array}{l}-- \\
-- \\
-- \\
-- \\
--\end{array}$ \\
\hline $\begin{array}{r}6 \ldots \ldots \ldots \\
7 \ldots \ldots \ldots \\
8 \ldots \ldots \ldots \\
9 \ldots \ldots \ldots \\
10 \ldots \ldots \ldots\end{array}$ & $\begin{array}{l}-- \\
237 \\
234 \\
207 \\
224\end{array}$ & $\begin{array}{l}-- \\
-- \\
-- \\
--\end{array}$ & $\begin{array}{l}-- \\
152 \\
142 \\
153 \\
138\end{array}$ & $\begin{array}{l}-- \\
35 \\
34 \\
37 \\
36\end{array}$ & $\begin{array}{r}-- \\
16 \\
13 \\
7 \\
5\end{array}$ & $\begin{array}{l}-- \\
8.5 \\
7.4 \\
6.3 \\
8.0\end{array}$ & $\begin{array}{r}-- \\
10.5 \\
9.2 \\
8.2 \\
9.1\end{array}$ & $\begin{array}{l}-- \\
27.9 \\
23.9 \\
22.8 \\
14.6\end{array}$ & $\begin{array}{r}-- \\
-2.8 \\
-2.4 \\
-1.8 \\
5.3\end{array}$ \\
\hline $\begin{array}{l}11 \ldots \ldots \ldots \\
12 \ldots \ldots \ldots \\
13 \ldots \ldots \ldots \\
14 \ldots \ldots \ldots \\
15 \ldots \ldots \ldots\end{array}$ & $\begin{array}{l}-- \\
238 \\
177 \\
183 \\
198\end{array}$ & $\begin{array}{l}-- \\
-- \\
-- \\
-- \\
--\end{array}$ & $\begin{array}{r}-- \\
81 \\
171 \\
157 \\
168\end{array}$ & $\begin{array}{l}-- \\
55 \\
31 \\
30 \\
27\end{array}$ & $\begin{array}{r}-- \\
-10 \\
45 \\
42 \\
54\end{array}$ & $\begin{array}{l}-- \\
-- \\
-- \\
-- \\
--\end{array}$ & $\begin{array}{r}-- \\
-1 \\
-2.5 \\
-.1 \\
1.6\end{array}$ & $\begin{array}{r}-- \\
7.3 \\
10.8 \\
9.0 \\
14.7\end{array}$ & $\begin{array}{r}-7.8 \\
-71.4 \\
-6.3 \\
-7.4\end{array}$ \\
\hline $\begin{array}{l}16 \ldots \ldots \ldots \\
17 \ldots \ldots \ldots \\
18 \ldots \ldots \ldots \\
19 \ldots \ldots \ldots \\
20 \ldots \ldots \ldots \ldots\end{array}$ & $\begin{array}{l}208 \\
230 \\
178 \\
168 \\
191\end{array}$ & $\begin{array}{l}-- \\
-- \\
-- \\
--\end{array}$ & $\begin{array}{l}124 \\
154 \\
151 \\
148 \\
138\end{array}$ & $\begin{array}{l}19 \\
33 \\
41 \\
40 \\
33\end{array}$ & $\begin{array}{l}29 \\
49 \\
21 \\
13 \\
20\end{array}$ & $\begin{array}{l}-- \\
-- \\
-- \\
-- \\
--\end{array}$ & $\begin{array}{r}3.4 \\
3.9 \\
1.3 \\
-1.0 \\
-1.1\end{array}$ & $\begin{array}{r}17.8 \\
18.1 \\
6.8 \\
12.7 \\
14.7\end{array}$ & $\begin{array}{r}-4.6 \\
-4.8 \\
-4.8 \\
-9.3 \\
-11.8\end{array}$ \\
\hline $\begin{array}{l}21 \ldots \ldots \ldots \\
22 \ldots \ldots \ldots \\
23 \ldots \ldots \\
24 \ldots \ldots \\
25 \ldots \ldots \ldots\end{array}$ & $\begin{array}{l}183 \\
202 \\
240 \\
268 \\
284\end{array}$ & $\begin{array}{l}-- \\
-- \\
-- \\
--\end{array}$ & $\begin{array}{r}146 \\
135 \\
60 \\
70 \\
25\end{array}$ & $\begin{array}{l}40 \\
35 \\
12 \\
15\end{array}$ & $\begin{array}{r}12 \\
8 \\
-11 \\
-2 \\
-17\end{array}$ & $\begin{array}{l}-- \\
-- \\
-- \\
--\end{array}$ & $\begin{array}{r}1.0 \\
.9 \\
3.5 \\
6.1 \\
7.7\end{array}$ & $\begin{array}{l}14.6 \\
14.6 \\
16.5 \\
16.1 \\
11.7\end{array}$ & $\begin{array}{r}-7.8 \\
-8.1 \\
-4.1 \\
-.9 \\
5.2\end{array}$ \\
\hline $\begin{array}{l}26 \ldots \ldots \ldots \\
27 \ldots \ldots \ldots \\
28 \ldots \ldots \ldots \\
29 \ldots \ldots \ldots \\
30 \ldots \ldots \ldots\end{array}$ & $\begin{array}{l}230 \\
244 \\
245 \\
260 \\
206\end{array}$ & $\begin{array}{l}-- \\
-- \\
-- \\
--\end{array}$ & $\begin{array}{r}136 \\
107 \\
77 \\
61 \\
131\end{array}$ & $\begin{array}{l}20 \\
18 \\
12 \\
13 \\
24\end{array}$ & $\begin{array}{r}49 \\
26 \\
11 \\
1 \\
25\end{array}$ & $\begin{array}{l}4.0 \\
3.6 \\
3.4 \\
4.8 \\
4.0\end{array}$ & $\begin{array}{l}6.4 \\
5.5 \\
5.5 \\
6.0 \\
5.7\end{array}$ & $\begin{array}{l}18.4 \\
15.6 \\
15.7 \\
14.7 \\
16.0\end{array}$ & $\begin{array}{r}-.2 \\
-1.1 \\
.1 \\
.9 \\
-1.4\end{array}$ \\
\hline Mean..... & $\overline{219}$ & $\overline{--}$ & $\overline{123}$ & $\overline{28}$ & $\overline{18}$ & 5.6 & 3.9 & $\overline{15.4}$ & -3.8 \\
\hline \multicolumn{10}{|c|}{ December 1985} \\
\hline $\begin{array}{l}1 \ldots \ldots \\
2 \ldots \ldots \\
3 \ldots \ldots \\
4 \ldots \ldots \\
5 \ldots \ldots\end{array}$ & $\begin{array}{l}238 \\
281 \\
266 \\
230 \\
238\end{array}$ & $\begin{array}{l}-- \\
-- \\
-- \\
-\overline{2}\end{array}$ & $\begin{array}{r}95 \\
4 \\
63 \\
131 \\
93\end{array}$ & $\begin{array}{r}16 \\
-2 \\
6 \\
19 \\
16\end{array}$ & $\begin{array}{r}17 \\
-27 \\
2 \\
38 \\
18\end{array}$ & $\begin{array}{l}2.5 \\
4.7 \\
5.7 \\
3.4 \\
3.4\end{array}$ & $\begin{array}{l}4.4 \\
6.0 \\
8.1 \\
5.8 \\
5.4\end{array}$ & $\begin{array}{r}14.3 \\
7.9 \\
17.2 \\
20.5 \\
17.9\end{array}$ & $\begin{array}{r}-2.3 \\
3.9 \\
.5 \\
-2.5 \\
-.5\end{array}$ \\
\hline $\begin{array}{r}6 \ldots \ldots \ldots \\
7 \ldots \ldots \\
8 \ldots \ldots \ldots \\
9 \ldots \ldots \ldots \\
10 \ldots \ldots \ldots\end{array}$ & $\begin{array}{r}221 \\
207 \\
184 \\
-- \\
--\end{array}$ & $\begin{array}{r}291 \\
286 \\
282 \\
-- \\
--\end{array}$ & $\begin{array}{r}129 \\
116 \\
130 \\
-- \\
--\end{array}$ & $\begin{array}{l}24 \\
24 \\
33 \\
-- \\
--\end{array}$ & $\begin{array}{l}29 \\
17 \\
11 \\
-- \\
--\end{array}$ & $\begin{array}{l}3.0 \\
2.9 \\
2.7 \\
-- \\
--\end{array}$ & $\begin{array}{l}5.1 \\
4.6 \\
4.3 \\
-- \\
--\end{array}$ & $\begin{array}{l}20.6 \\
17.1 \\
11.4 \\
-- \\
--\end{array}$ & $\begin{array}{l}-3.8 \\
-2.7 \\
-5.0 \\
-- \\
--\end{array}$ \\
\hline $\begin{array}{l}11 \ldots \ldots \ldots \\
12 \ldots \ldots \ldots \\
13 \ldots \ldots \ldots \\
14 \ldots \ldots \ldots \\
15 \ldots \ldots \ldots\end{array}$ & $\begin{array}{l}-- \\
183 \\
199 \\
199\end{array}$ & $\begin{array}{r}-- \\
268 \\
274 \\
278\end{array}$ & $\begin{array}{l}-- \\
-- \\
120 \\
120 \\
112\end{array}$ & $\begin{array}{l}-- \\
-- \\
28 \\
28 \\
24\end{array}$ & $\begin{array}{r}-- \\
-11 \\
13 \\
9\end{array}$ & $\begin{array}{r}-- \\
-- \\
-1.6 \\
-.5 \\
2.2\end{array}$ & $\begin{array}{l}-- \\
-- \\
.3 \\
2.1 \\
4.3\end{array}$ & $\begin{array}{l}-- \\
\overline{15.4} \\
18.8 \\
21.6\end{array}$ & $\begin{array}{r}-- \\
-10.3 \\
-8.7 \\
-7.6\end{array}$ \\
\hline $\begin{array}{l}16 \ldots \ldots \ldots \\
17 \ldots \ldots \ldots \\
18 \ldots \ldots \ldots \\
19 \ldots \ldots \ldots \\
20 \ldots \ldots \ldots\end{array}$ & $\begin{array}{l}197 \\
198 \\
222 \\
217 \\
210\end{array}$ & $\begin{array}{l}288 \\
295 \\
303 \\
293 \\
277\end{array}$ & $\begin{array}{l}123 \\
124 \\
125 \\
123 \\
127\end{array}$ & $\begin{array}{l}30 \\
30 \\
33 \\
31 \\
33\end{array}$ & $\begin{array}{r}5 \\
4 \\
12 \\
14 \\
21\end{array}$ & $\begin{array}{l}5.1 \\
6.1 \\
7.3 \\
4.4 \\
-.4\end{array}$ & $\begin{array}{r}6.6 \\
7.5 \\
9.1 \\
6.1 \\
.3\end{array}$ & $\begin{array}{l}17.9 \\
19.3 \\
23.9 \\
26.6 \\
24.6\end{array}$ & $\begin{array}{l}-2.3 \\
-2.7 \\
-2.5 \\
-6.5 \\
-6.3\end{array}$ \\
\hline
\end{tabular}


Table 1. Radiation and temperature field data for undisturbed-land-surface site, May 1985 through June 1986 -Continued

\begin{tabular}{|c|c|c|c|c|c|c|c|c|c|}
\hline \multirow{3}{*}{ Date } & \multicolumn{5}{|c|}{$\begin{array}{l}\text { Daily mean radiation } \\
\text { (watts per square meter) }\end{array}$} & \multicolumn{4}{|c|}{$\begin{array}{c}\text { Temperature } \\
\text { (degrees Celsius) }\end{array}$} \\
\hline & \multicolumn{2}{|c|}{ Long wave } & \multicolumn{2}{|c|}{ Short wave } & \multirow{2}{*}{ Net } & \multirow{2}{*}{$\begin{array}{c}\text { Daily mean } \\
\text { air } \\
\text { temperature }\end{array}$} & \multirow{2}{*}{$\frac{\text { Soil }}{\text { Mean }}$} & \multirow{2}{*}{$\frac{\text { surface } t \epsilon}{\text { Maximum }}$} & Cemperature \\
\hline & In & out & In & $\overline{\text { Out }}$ & & & & & Minimum \\
\hline \multicolumn{10}{|c|}{ December 1985-Continued } \\
\hline $\begin{array}{l}21 \ldots \ldots \ldots \\
22 \ldots \ldots \\
23 \ldots \ldots \\
24 \ldots \ldots \\
25 \ldots \ldots\end{array}$ & $\begin{array}{l}-- \\
-- \\
209 \\
212 \\
211\end{array}$ & $\begin{array}{l}-- \\
-- \\
292 \\
306 \\
308\end{array}$ & $\begin{array}{r}-- \\
-- \\
121 \\
121 \\
115\end{array}$ & $\begin{array}{l}-- \\
-- \\
31 \\
30 \\
27\end{array}$ & $\begin{array}{r}-- \\
-- \\
-4 \\
-12\end{array}$ & $\begin{array}{l}-- \\
-- \\
5.1 \\
9.0 \\
8.7\end{array}$ & $\begin{array}{c}-- \\
-- \\
6.3 \\
10.3 \\
10.6\end{array}$ & $\begin{array}{c}-- \\
-- \\
23.3 \\
22.2 \\
24.6\end{array}$ & $\begin{array}{r}-- \\
-- \\
-5.6 \\
1.5 \\
-.6\end{array}$ \\
\hline $\begin{array}{l}26 \ldots \ldots \ldots \\
27 \ldots \ldots \ldots \\
28 \ldots \ldots \ldots \\
29 \ldots \ldots \ldots \\
30 \ldots \ldots \ldots\end{array}$ & $\begin{array}{l}201 \\
196 \\
199 \\
270 \\
275\end{array}$ & $\begin{array}{l}291 \\
287 \\
286 \\
298 \\
309\end{array}$ & $\begin{array}{r}120 \\
122 \\
118 \\
55 \\
52\end{array}$ & $\begin{array}{r}27 \\
29 \\
28 \\
8 \\
9\end{array}$ & $\begin{array}{r}-7 \\
-8 \\
-5 \\
-7 \\
-13\end{array}$ & $\begin{array}{l}2.9 \\
1.9 \\
1.4 \\
3.6 \\
6.5\end{array}$ & $\begin{array}{l}5.1 \\
4.0 \\
3.3 \\
5.6 \\
8.5\end{array}$ & $\begin{array}{l}24.7 \\
23.3 \\
21.1 \\
15.0 \\
18.8\end{array}$ & $\begin{array}{r}-6.5 \\
-7.6 \\
-7.2 \\
-2.6 \\
1.9\end{array}$ \\
\hline $31 \ldots \ldots \ldots$ & 235 & 294 & 78 & 15 & -5 & 3.6 & 5.8 & 19.6 & -2.0 \\
\hline Mean..... & $\overline{220}$ & $\overline{290}$ & $\overline{105}$ & $\overline{24}$ & $\overline{5}$ & $\overline{3.7}$ & 5.6 & $\overline{19.5}$ & -3.5 \\
\hline \multicolumn{10}{|c|}{ January 1986} \\
\hline $\begin{array}{l}1 \ldots \ldots \\
2 \ldots \ldots \\
3 \ldots \ldots \\
4 \ldots \ldots \\
5 \ldots \ldots\end{array}$ & $\begin{array}{l}215 \\
231 \\
217 \\
277 \\
284\end{array}$ & $\begin{array}{l}287 \\
287 \\
294 \\
314 \\
310\end{array}$ & $\begin{array}{r}107 \\
93 \\
129 \\
83 \\
39\end{array}$ & $\begin{array}{r}24 \\
18 \\
28 \\
17 \\
4\end{array}$ & $\begin{array}{r}6 \\
2 \\
11 \\
3 \\
-13\end{array}$ & $\begin{array}{l}1.8 \\
1.9 \\
3.3 \\
7.2 \\
6.5\end{array}$ & $\begin{array}{l}3.9 \\
3.9 \\
5.8 \\
9.3 \\
8.6\end{array}$ & $\begin{array}{l}19.8 \\
16.5 \\
22.6 \\
19.2 \\
15.0\end{array}$ & $\begin{array}{r}-5.6 \\
-5.4 \\
-4.8 \\
2.3 \\
3.1\end{array}$ \\
\hline $\begin{array}{c}6 \ldots \ldots \ldots \\
7 \ldots \ldots \\
8 \ldots \ldots \ldots \\
9 \ldots \ldots \ldots \\
10 \ldots \ldots\end{array}$ & $\begin{array}{l}221 \\
181 \\
186 \\
201 \\
207\end{array}$ & $\begin{array}{l}290 \\
280 \\
274 \\
290 \\
297\end{array}$ & $\begin{array}{l}125 \\
132 \\
118 \\
148 \\
125\end{array}$ & $\begin{array}{l}29 \\
33 \\
26 \\
33 \\
28\end{array}$ & $\begin{array}{r}19 \\
4 \\
-2 \\
17 \\
0\end{array}$ & $\begin{array}{l}-- \\
-- \\
-- \\
--\end{array}$ & $\begin{array}{l}6.2 \\
4.5 \\
1.9 \\
5.5 \\
7.5\end{array}$ & $\begin{array}{l}16.3 \\
15.0 \\
21.7 \\
26.9 \\
28.7\end{array}$ & $\begin{array}{r}-.4 \\
-5.7 \\
-9.5 \\
-7.2 \\
-4.5\end{array}$ \\
\hline $\begin{array}{l}11 \ldots \ldots \ldots \\
12 \ldots \ldots \\
13 \ldots \ldots \\
14 \ldots \ldots \\
15 \ldots \ldots\end{array}$ & $\begin{array}{r}209 \\
240 \\
-- \\
248 \\
261\end{array}$ & $\begin{array}{r}298 \\
299 \\
-- \\
294 \\
305\end{array}$ & $\begin{array}{r}125 \\
129 \\
-- \\
58 \\
66\end{array}$ & $\begin{array}{l}28 \\
29 \\
-- \\
10 \\
13\end{array}$ & $\begin{array}{r}-3 \\
-1 \\
-- \\
-18 \\
-8\end{array}$ & $\begin{array}{l}-- \\
-- \\
-- \\
-- \\
--\end{array}$ & $\begin{array}{l}7.4 \\
7.1 \\
-- \\
5.5 \\
7.5\end{array}$ & $\begin{array}{c}27.5 \\
28.0 \\
-- \\
17.2 \\
14.6\end{array}$ & $\begin{array}{c}-4.5 \\
-5.0 \\
-- \\
-2.9 \\
1.2\end{array}$ \\
\hline $\begin{array}{l}16 \ldots \ldots \ldots \\
17 \ldots \ldots \ldots \\
18 \ldots \ldots \ldots \\
19 \ldots \ldots \\
20 \ldots \ldots\end{array}$ & $\begin{array}{l}226 \\
221 \\
222 \\
223 \\
205\end{array}$ & $\begin{array}{l}293 \\
294 \\
307 \\
302 \\
302\end{array}$ & $\begin{array}{r}95 \\
118 \\
132 \\
116 \\
140\end{array}$ & $\begin{array}{l}20 \\
26 \\
29 \\
26 \\
35\end{array}$ & $\begin{array}{r}-3 \\
9 \\
5 \\
0 \\
2\end{array}$ & $\begin{array}{l}-- \\
-- \\
-- \\
-- \\
--\end{array}$ & $\begin{array}{l}5.2 \\
5.3 \\
9.0 \\
7.6 \\
8.6\end{array}$ & $\begin{array}{l}18.6 \\
20.8 \\
28.7 \\
24.9 \\
20.7\end{array}$ & $\begin{array}{r}-2.0 \\
-4.5 \\
-1.8 \\
-3.9 \\
.0\end{array}$ \\
\hline $\begin{array}{l}21 \ldots \ldots \ldots \\
22 \ldots \ldots \ldots \\
23 \ldots \ldots \\
24 \ldots \ldots \\
25 \ldots \ldots \ldots\end{array}$ & $\begin{array}{l}-- \\
-- \\
199 \\
204\end{array}$ & $\begin{array}{r}-- \\
-- \\
-- \\
304 \\
302\end{array}$ & $\begin{array}{l}-- \\
-- \\
-- \\
164 \\
151\end{array}$ & $\begin{array}{l}-- \\
-- \\
-- \\
41 \\
38\end{array}$ & $\begin{array}{l}-- \\
-- \\
-- \\
13 \\
7\end{array}$ & $\begin{array}{l}-- \\
-- \\
-- \\
-- \\
--\end{array}$ & $\begin{array}{l}-- \\
-- \\
-- \\
8.3 \\
7.7\end{array}$ & $\begin{array}{c}-- \\
-- \\
-- \\
21.5 \\
23.2\end{array}$ & $\begin{array}{c}-- \\
-- \\
-- \\
-.8 \\
-3.3\end{array}$ \\
\hline $\begin{array}{l}26 \ldots \ldots \ldots \\
27 \ldots \ldots \ldots \\
28 \ldots \ldots \\
29 \ldots \ldots \ldots \\
30 \ldots \ldots\end{array}$ & $\begin{array}{l}205 \\
205 \\
209 \\
227 \\
291\end{array}$ & $\begin{array}{l}299 \\
301 \\
302 \\
305 \\
308\end{array}$ & $\begin{array}{r}151 \\
151 \\
151 \\
134 \\
16\end{array}$ & $\begin{array}{l}36 \\
36 \\
37 \\
32 \\
--\end{array}$ & $\begin{array}{r}6 \\
-6 \\
-7 \\
-6 \\
-27\end{array}$ & $\begin{array}{l}-- \\
-- \\
4.2 \\
5.3 \\
7.0\end{array}$ & $\begin{array}{l}7.0 \\
6.7 \\
6.9 \\
7.2 \\
8.6\end{array}$ & $\begin{array}{l}27.8 \\
28.3 \\
25.3 \\
23.8 \\
11.3\end{array}$ & $\begin{array}{r}-7.2 \\
-6.7 \\
-5.9 \\
-5.2 \\
6.8\end{array}$ \\
\hline $31 \ldots \ldots \ldots$ & 271 & 316 & 85 & 9 & 0 & 8.5 & 10.2 & 17.0 & 7.3 \\
\hline Mean..... & $\overline{225}$ & $\overline{298}$ & $\overline{114}$ & $\overline{26}$ & $\overline{0}$ & $\overline{5.1}$ & 6.8 & $\overline{21.5}$ & -2.8 \\
\hline
\end{tabular}


Table 1. Radiation and temperature field data for undisturbed-land-surface site, May 1985 through June 1986 - Continued

\begin{tabular}{|c|c|c|c|c|c|c|c|c|c|}
\hline \multirow{3}{*}{ Date } & \multicolumn{5}{|c|}{$\begin{array}{l}\text { Daily mean radiation } \\
\text { (watts per square meter) }\end{array}$} & \multicolumn{4}{|c|}{$\begin{array}{l}\text { Temperature } \\
\text { (degrees Celsius) }\end{array}$} \\
\hline & \multicolumn{2}{|c|}{ Long wave } & \multicolumn{2}{|c|}{ Short wave } & \multirow{2}{*}{ Net } & \multirow{2}{*}{$\begin{array}{c}\text { Daily mean } \\
\text { air } \\
\text { temperature }\end{array}$} & \multirow{2}{*}{ Soil } & surface & temperature \\
\hline & In & $\overline{\text { Out }}$ & In & $\overline{\text { out }}$ & & & & Maximum & Minimum \\
\hline \multicolumn{10}{|c|}{ Fobruary 1986} \\
\hline $\begin{array}{l}1 \ldots \ldots \\
2 \ldots \ldots \\
3 \ldots \ldots \\
4 \ldots \ldots \\
5 \ldots \ldots\end{array}$ & $\begin{array}{l}232 \\
216 \\
200 \\
-- \\
--\end{array}$ & $\begin{array}{l}306 \\
292 \\
295 \\
-- \\
--\end{array}$ & $\begin{array}{r}146 \\
157 \\
185 \\
-- \\
--\end{array}$ & $\begin{array}{l}19 \\
26 \\
29 \\
-- \\
--\end{array}$ & $\begin{array}{l}18 \\
20 \\
33 \\
-- \\
--\end{array}$ & $\begin{array}{l}7.1 \\
4.6 \\
3.5 \\
-- \\
--\end{array}$ & $\begin{array}{l}10.0 \\
6.6 \\
5.8 \\
-- \\
--\end{array}$ & $\begin{array}{c}22.4 \\
16.1 \\
18.3 \\
-- \\
--\end{array}$ & $\begin{array}{r}2.3 \\
-.8 \\
-2.2 \\
-- \\
--\end{array}$ \\
\hline $\begin{array}{c}6 \ldots \ldots \ldots \\
7 \ldots \ldots \ldots \\
8 \ldots \ldots \ldots \\
9 \ldots \ldots \ldots \\
10 \ldots \ldots\end{array}$ & $\begin{array}{l}-- \\
-- \\
-- \\
-- \\
164\end{array}$ & $\begin{array}{c}-- \\
-- \\
-- \\
-- \\
267\end{array}$ & $\begin{array}{c}-- \\
-- \\
-- \\
-- \\
166\end{array}$ & $\begin{array}{l}-- \\
-- \\
-- \\
-- \\
37\end{array}$ & $\begin{array}{l}-- \\
-- \\
-- \\
-- \\
-4\end{array}$ & $\begin{array}{l}-- \\
-- \\
-- \\
-- \\
-2.0\end{array}$ & $\begin{array}{l}-- \\
-- \\
-- \\
-- \\
.0\end{array}$ & $\begin{array}{c}-- \\
-- \\
-- \\
-\overline{12.4}\end{array}$ & $\begin{array}{c}-- \\
-- \\
-- \\
-- \\
-11.0\end{array}$ \\
\hline $\begin{array}{l}11 \ldots \ldots \\
12 \ldots \ldots \\
13 \ldots \ldots \\
14 \ldots \ldots \ldots \\
15 \ldots \ldots\end{array}$ & $\begin{array}{l}201 \\
243 \\
269 \\
280 \\
270\end{array}$ & $\begin{array}{l}261 \\
270 \\
306 \\
309 \\
311\end{array}$ & $\begin{array}{r}88 \\
40 \\
93 \\
30 \\
116\end{array}$ & $\begin{array}{r}18 \\
4 \\
16 \\
2 \\
17\end{array}$ & $\begin{array}{r}-24 \\
-26 \\
0 \\
-31 \\
17\end{array}$ & $\begin{array}{r}-2.3 \\
.1 \\
7.5 \\
8.3 \\
8.3\end{array}$ & $\begin{array}{l}-.5 \\
1.9 \\
9.1 \\
9.8 \\
9.6\end{array}$ & $\begin{array}{r}11.0 \\
9.3 \\
16.2 \\
16.0 \\
15.7\end{array}$ & $\begin{array}{r}-9.3 \\
-5.7 \\
4.6 \\
7.1 \\
7.1\end{array}$ \\
\hline $\begin{array}{l}16 \ldots \ldots \\
17 \ldots \ldots \\
18 \ldots \ldots \ldots \\
19 \ldots \ldots \ldots \\
20 \ldots \ldots\end{array}$ & $\begin{array}{l}235 \\
244 \\
271 \\
259 \\
200\end{array}$ & $\begin{array}{l}304 \\
305 \\
319 \\
314 \\
298\end{array}$ & $\begin{array}{r}109 \\
95 \\
94 \\
79 \\
172\end{array}$ & $\begin{array}{l}15 \\
14 \\
15 \\
12 \\
36\end{array}$ & $\begin{array}{r}-3 \\
-11 \\
-8 \\
-23 \\
-2\end{array}$ & $\begin{array}{r}8.2 \\
8.5 \\
12.0 \\
11.8 \\
5.9\end{array}$ & $\begin{array}{r}9.6 \\
9.8 \\
13.4 \\
12.9 \\
8.7\end{array}$ & $\begin{array}{l}16.1 \\
18.3 \\
22.5 \\
17.6 \\
22.5\end{array}$ & $\begin{array}{r}3.6 \\
1.8 \\
8.4 \\
6.3 \\
-2.2\end{array}$ \\
\hline $\begin{array}{l}21 \ldots \ldots \ldots \\
22 \ldots \ldots \ldots \\
23 \ldots \ldots \ldots \\
24 \ldots \ldots \\
25 \ldots \ldots \ldots\end{array}$ & $\begin{array}{l}195 \\
209 \\
228 \\
232 \\
--\end{array}$ & $\begin{array}{c}302 \\
305 \\
322 \\
306 \\
--\end{array}$ & $\begin{array}{l}197 \\
183 \\
191 \\
146 \\
--\end{array}$ & $\begin{array}{l}45 \\
41 \\
43 \\
35 \\
--\end{array}$ & $\begin{array}{r}5 \\
6 \\
11 \\
-1 \\
--\end{array}$ & $\begin{array}{r}6.0 \\
5.8 \\
10.0 \\
9.3 \\
--\end{array}$ & $\begin{array}{r}8.9 \\
8.4 \\
12.3 \\
10.7 \\
--\end{array}$ & $\begin{array}{l}25.8 \\
24.6 \\
31.9 \\
28.6 \\
--\end{array}$ & $\begin{array}{l}-2.6 \\
-2.9 \\
-1.2 \\
2.8 \\
--\end{array}$ \\
\hline $\begin{array}{l}26 \ldots \ldots \ldots \\
27 \ldots \ldots \ldots \\
28 \ldots \ldots\end{array}$ & $\begin{array}{l}240 \\
245 \\
230\end{array}$ & $\begin{array}{l}346 \\
346 \\
340\end{array}$ & $\begin{array}{l}203 \\
205 \\
207\end{array}$ & $\begin{array}{l}46 \\
50 \\
49\end{array}$ & $\begin{array}{l}9 \\
10 \\
10\end{array}$ & $\begin{array}{l}13.9 \\
14.7 \\
12.6\end{array}$ & $\begin{array}{l}-- \\
-- \\
--\end{array}$ & $\begin{array}{l}-- \\
--\end{array}$ & $\begin{array}{l}-- \\
-- \\
--\end{array}$ \\
\hline Mean..... & $\overline{232}$ & $\overline{306}$ & $\overline{138}$ & 27 & $\overline{0}$ & 7.3 & 8.2 & $\overline{19.2}$ & .3 \\
\hline \multicolumn{10}{|c|}{ March 1986} \\
\hline $\begin{array}{l}1 \ldots \ldots \\
2 \ldots \ldots \\
3 \ldots \ldots \\
4 \ldots \ldots \\
5 \ldots \ldots\end{array}$ & $\begin{array}{l}243 \\
270 \\
260 \\
242 \\
242\end{array}$ & $\begin{array}{l}340 \\
322 \\
343 \\
344 \\
347\end{array}$ & $\begin{array}{r}197 \\
88 \\
202 \\
207 \\
217\end{array}$ & $\begin{array}{l}47 \\
16 \\
49 \\
49 \\
51\end{array}$ & $\begin{array}{r}12 \\
-20 \\
18 \\
11 \\
15\end{array}$ & $\begin{array}{l}12.0 \\
10.5 \\
12.9 \\
13.0 \\
12.6\end{array}$ & $\begin{array}{l}14.6 \\
12.1 \\
15.7 \\
16.4 \\
16.0\end{array}$ & $\begin{array}{l}31.9 \\
23.8 \\
31.0 \\
35.1 \\
36.0\end{array}$ & $\begin{array}{r}0.1 \\
5.5 \\
5.8 \\
4.3 \\
.5\end{array}$ \\
\hline $\begin{array}{r}6 \ldots \ldots \ldots \\
7 \ldots \ldots \ldots \\
8 \ldots \ldots \ldots \\
9 \ldots \ldots \ldots \\
10 \ldots \ldots \ldots\end{array}$ & $\begin{array}{l}244 \\
252 \\
272 \\
239 \\
284\end{array}$ & $\begin{array}{l}341 \\
331 \\
320 \\
308 \\
290\end{array}$ & $\begin{array}{r}202 \\
152 \\
104 \\
204 \\
36\end{array}$ & $\begin{array}{r}48 \\
35 \\
22 \\
45 \\
0\end{array}$ & $\begin{array}{r}14 \\
1 \\
-5 \\
34 \\
-19\end{array}$ & $\begin{array}{r}11.3 \\
10.2 \\
10.8 \\
7.2 \\
5.1\end{array}$ & $\begin{array}{r}14.2 \\
12.7 \\
12.1 \\
9.5 \\
6.5\end{array}$ & $\begin{array}{r}30.6 \\
27.4 \\
20.1 \\
18.6 \\
9.6\end{array}$ & $\begin{array}{l}1.1 \\
2.6 \\
5.6 \\
-.7 \\
4.1\end{array}$ \\
\hline $\begin{array}{l}11 \ldots \ldots \ldots \\
12 \ldots \ldots \\
13 \ldots \ldots \\
14 \ldots \ldots \\
15 \ldots \ldots \ldots\end{array}$ & $\begin{array}{l}233 \\
218 \\
233 \\
226 \\
222\end{array}$ & $\begin{array}{l}295 \\
285 \\
284 \\
292 \\
288\end{array}$ & $\begin{array}{l}200 \\
120 \\
151 \\
223 \\
211\end{array}$ & $\begin{array}{l}32 \\
19 \\
29 \\
32 \\
39\end{array}$ & $\begin{array}{l}51 \\
-5 \\
15 \\
54 \\
50\end{array}$ & $\begin{array}{l}6.6 \\
5.0 \\
3.5 \\
5.1 \\
3.9\end{array}$ & $\begin{array}{l}8.9 \\
6.7 \\
6.1 \\
8.4 \\
6.2\end{array}$ & $\begin{array}{l}20.1 \\
16.4 \\
18.8 \\
22.1 \\
18.6\end{array}$ & $\begin{array}{r}.9 \\
.9 \\
-2.3 \\
.9 \\
-3.1\end{array}$ \\
\hline $\begin{array}{l}16 \ldots \ldots \\
17 \ldots \ldots \\
18 \ldots \ldots \\
19 \ldots \ldots \\
20 \ldots \ldots\end{array}$ & $\begin{array}{r}244 \\
215 \\
-- \\
-- \\
214\end{array}$ & $\begin{array}{r}285 \\
259 \\
-- \\
-- \\
319\end{array}$ & $\begin{array}{r}130 \\
87 \\
-- \\
-- \\
252\end{array}$ & $\begin{array}{l}15 \\
11 \\
-- \\
-- \\
56\end{array}$ & $\begin{array}{r}19 \\
-16 \\
-- \\
-- \\
28\end{array}$ & $\begin{array}{l}4.0 \\
1.3 \\
-- \\
-- \\
9.7\end{array}$ & $\begin{array}{c}5.9 \\
2.6 \\
-- \\
-- \\
13.6\end{array}$ & $\begin{array}{c}13.7 \\
11.7 \\
-- \\
-- \\
32.8\end{array}$ & $\begin{array}{l}2.5 \\
-.5 \\
-- \\
-- \\
.6\end{array}$ \\
\hline
\end{tabular}


Table 1. Radiation and temperature field data for undisturbed-land-surface site, May 1985 through June $1986-$ Continued

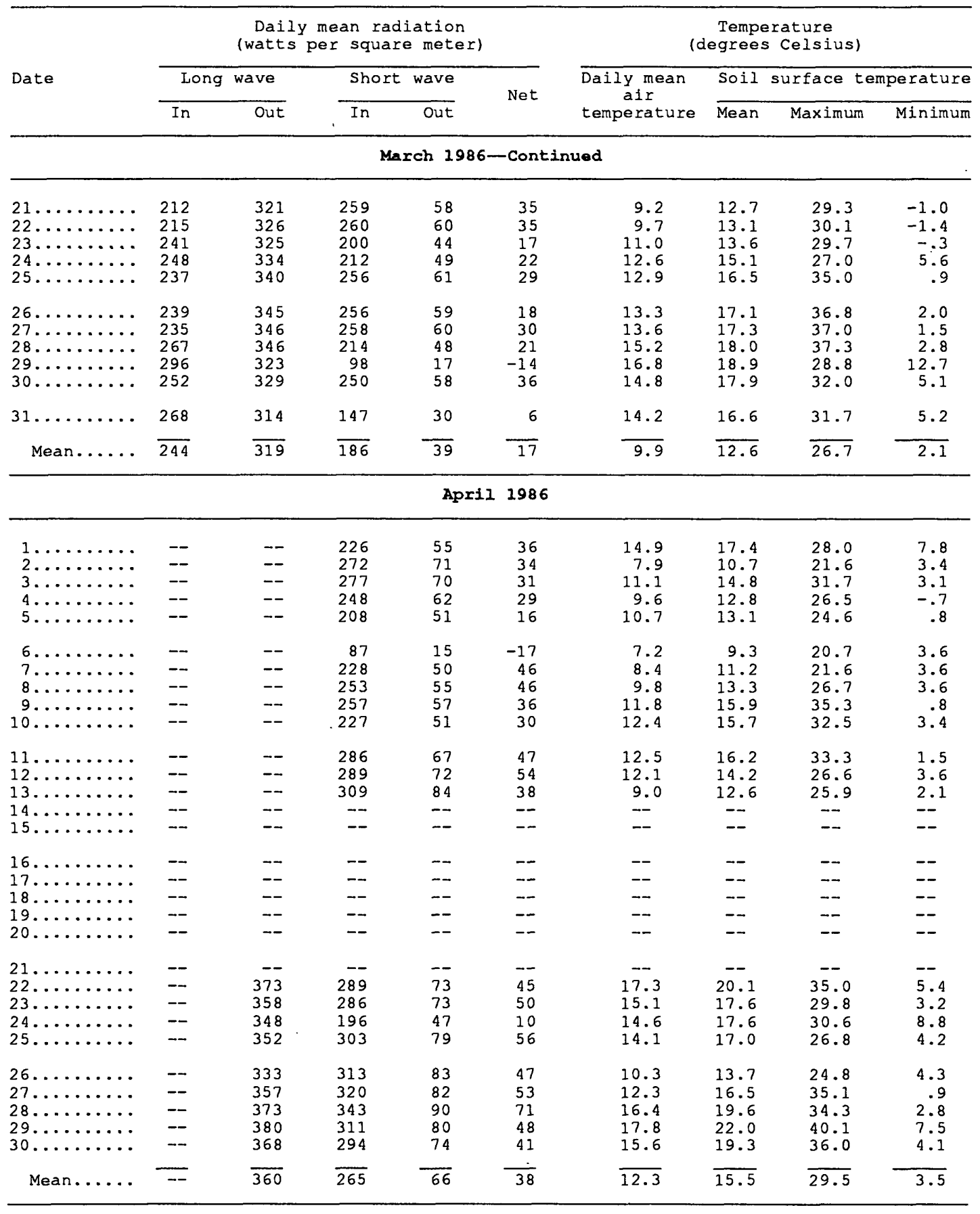


Table 1. Radiation and temperature field data for undisturbed-land-surface site, May 1985 through June 1986 -Continued

\begin{tabular}{|c|c|c|c|c|c|c|c|c|c|}
\hline \multirow{3}{*}{ Date } & \multicolumn{5}{|c|}{$\begin{array}{l}\text { Daily mean radiation } \\
\text { (watts per square meter) }\end{array}$} & \multicolumn{4}{|c|}{$\begin{array}{l}\text { Temperature } \\
\text { (degrees Celsius) }\end{array}$} \\
\hline & Long & wave & \multicolumn{2}{|c|}{ Short wave } & \multirow{2}{*}{ Net } & \multirow{2}{*}{$\begin{array}{c}\text { Daily mean } \\
\text { air } \\
\text { temperature }\end{array}$} & \multirow{2}{*}{ Soil } & \multirow{2}{*}{$\begin{array}{c}\text { surface } t \\
\text { Maximum }\end{array}$} & temperature \\
\hline & In & Out & In & Out & & & & & Minimum \\
\hline \multicolumn{10}{|c|}{ May 1986} \\
\hline $\begin{array}{l}1 \ldots \ldots \\
2 \ldots \ldots \\
3 \ldots \ldots \\
4 \ldots \ldots \\
5 \ldots \ldots\end{array}$ & $\begin{array}{l}-- \\
-- \\
-- \\
-- \\
--\end{array}$ & $\begin{array}{l}370 \\
375 \\
368 \\
355 \\
347\end{array}$ & $\begin{array}{l}263 \\
331 \\
333 \\
301 \\
263\end{array}$ & $\begin{array}{l}67 \\
88 \\
90 \\
79 \\
67\end{array}$ & $\begin{array}{l}37 \\
62 \\
70 \\
47 \\
40\end{array}$ & $\begin{array}{l}17.3 \\
17.7 \\
17.8 \\
12.8 \\
11.7\end{array}$ & $\begin{array}{l}20.2 \\
20.8 \\
20.1 \\
16.5 \\
14.7\end{array}$ & $\begin{array}{l}33.6 \\
35.2 \\
31.0 \\
28.3 \\
27.6\end{array}$ & $\begin{array}{l}5.8 \\
6.2 \\
7.5 \\
6.2 \\
-.5\end{array}$ \\
\hline $\begin{array}{c}6 \ldots \ldots \ldots \\
7 \ldots \ldots \\
8 \ldots \ldots \ldots \\
9 \ldots \ldots \ldots \\
10 \ldots \ldots\end{array}$ & $\begin{array}{l}-- \\
-- \\
-- \\
-- \\
--\end{array}$ & $\begin{array}{l}303 \\
331 \\
337 \\
355 \\
356\end{array}$ & $\begin{array}{r}73 \\
335 \\
289 \\
338 \\
324\end{array}$ & $\begin{array}{r}8 \\
82 \\
69 \\
82 \\
80\end{array}$ & $\begin{array}{r}-16 \\
80 \\
44 \\
58 \\
66\end{array}$ & $\begin{array}{r}6.7 \\
9.9 \\
11.3 \\
13.0 \\
13.8\end{array}$ & $\begin{array}{r}8.9 \\
13.6 \\
14.7 \\
17.3 \\
18.2\end{array}$ & $\begin{array}{l}14.9 \\
28.6 \\
28.8 \\
34.3 \\
30.9\end{array}$ & $\begin{array}{l}5.3 \\
2.4 \\
2.3 \\
2.0 \\
4.8\end{array}$ \\
\hline $\begin{array}{l}11 \ldots \ldots \ldots \\
12 \ldots \ldots \ldots \\
13 \ldots \ldots \ldots \\
14 \ldots \ldots \ldots \\
15 \ldots \ldots \ldots\end{array}$ & $\begin{array}{l}-- \\
-- \\
-- \\
-- \\
--\end{array}$ & $\begin{array}{l}365 \\
378 \\
387 \\
390 \\
402\end{array}$ & $\begin{array}{l}334 \\
335 \\
327 \\
297 \\
311\end{array}$ & $\begin{array}{l}83 \\
82 \\
80 \\
71 \\
76\end{array}$ & $\begin{array}{l}61 \\
54 \\
58 \\
52 \\
50\end{array}$ & $\begin{array}{c}-- \\
-- \\
18.3 \\
19.4 \\
21.2\end{array}$ & $\begin{array}{l}18.9 \\
21.0 \\
22.4 \\
22.8 \\
25.0\end{array}$ & $\begin{array}{l}32.1 \\
40.1 \\
38.6 \\
38.7 \\
40.2\end{array}$ & $\begin{array}{r}4.0 \\
3.3 \\
6.6 \\
7.8 \\
16.2\end{array}$ \\
\hline $\begin{array}{l}16 \ldots \ldots \ldots \\
17 \ldots \ldots \ldots \\
18 \ldots \ldots \ldots \\
19 \ldots \ldots \ldots \\
20 \ldots \ldots \ldots\end{array}$ & $\begin{array}{l}-- \\
-- \\
-- \\
-- \\
--\end{array}$ & $\begin{array}{r}389 \\
386 \\
-- \\
--\end{array}$ & $\begin{array}{r}343 \\
351 \\
-- \\
-- \\
325\end{array}$ & $\begin{array}{l}86 \\
87 \\
-- \\
-- \\
84\end{array}$ & $\begin{array}{l}52 \\
56 \\
-- \\
-- \\
68\end{array}$ & $\begin{array}{c}19.3 \\
17.3 \\
-- \\
\overline{21.7}\end{array}$ & $\begin{array}{c}23.0 \\
22.0 \\
-- \\
-- \\
24.3\end{array}$ & $\begin{array}{c}37.4 \\
40.2 \\
-- \\
-- \\
41.5\end{array}$ & $\begin{array}{c}10.7 \\
6.6 \\
-- \\
-- \\
3.4\end{array}$ \\
\hline $\begin{array}{l}21 \ldots \ldots \ldots \\
22 \ldots \ldots \ldots \\
23 \ldots \ldots \ldots \\
24 \ldots \ldots \\
25 \ldots \ldots \ldots\end{array}$ & $\begin{array}{l}-- \\
-- \\
-- \\
--\end{array}$ & $\begin{array}{l}394 \\
381 \\
390 \\
405 \\
416\end{array}$ & $\begin{array}{l}338 \\
324 \\
312 \\
332 \\
339\end{array}$ & $\begin{array}{l}89 \\
84 \\
80 \\
85 \\
85\end{array}$ & $\begin{array}{l}75 \\
53 \\
58 \\
58 \\
60\end{array}$ & $\begin{array}{l}20.6 \\
16.9 \\
17.2 \\
20.3 \\
22.4\end{array}$ & $\begin{array}{l}23.1 \\
21.0 \\
21.0 \\
24.6 \\
26.5\end{array}$ & $\begin{array}{l}32.3 \\
39.6 \\
36.3 \\
43.4 \\
45.7\end{array}$ & $\begin{array}{r}15.1 \\
9.0 \\
3.0 \\
5.9 \\
7.1\end{array}$ \\
\hline $\begin{array}{l}26 \ldots \ldots \ldots \\
27 \ldots \ldots \ldots \\
28 \ldots \ldots \ldots \\
29 \ldots \ldots \ldots \\
30 \ldots \ldots \ldots\end{array}$ & $\begin{array}{l}-- \\
-- \\
-- \\
--\end{array}$ & $\begin{array}{l}415 \\
417 \\
414 \\
420 \\
411\end{array}$ & $\begin{array}{l}338 \\
336 \\
306 \\
286 \\
338\end{array}$ & $\begin{array}{l}86 \\
84 \\
77 \\
67 \\
82\end{array}$ & $\begin{array}{l}63 \\
60 \\
58 \\
38 \\
68\end{array}$ & $\begin{array}{l}23.1 \\
24.0 \\
24.5 \\
25.6 \\
24.2\end{array}$ & $\begin{array}{l}26.6 \\
27.7 \\
27.6 \\
29.5 \\
27.3\end{array}$ & $\begin{array}{l}44.5 \\
44.7 \\
43.8 \\
48.9 \\
46.5\end{array}$ & $\begin{array}{r}8.6 \\
10.6 \\
12.0 \\
12.2 \\
13.9\end{array}$ \\
\hline $31 \ldots \ldots \ldots$ & -- & -- & -- & -- & -- & -- & -- & -- & -- \\
\hline Mean..... & $\overline{--}$ & $\overline{381}$ & $\overline{312}$ & 78 & $\overrightarrow{55}$ & $\overline{18.0}$ & $\overline{21.4}$ & $\overline{36.7}$ & 7.1 \\
\hline \multicolumn{10}{|c|}{ June 1986} \\
\hline $\begin{array}{l}1 \ldots \ldots \ldots \\
2 \ldots \ldots \ldots \\
3 \ldots \ldots \\
4 \ldots \ldots \\
5 \ldots \ldots\end{array}$ & $\begin{array}{l}-- \\
-- \\
-- \\
--\end{array}$ & $\begin{array}{l}447 \\
430 \\
428 \\
414 \\
405\end{array}$ & $\begin{array}{l}330 \\
290 \\
336 \\
297 \\
358\end{array}$ & $\begin{array}{l}78 \\
70 \\
81 \\
70 \\
90\end{array}$ & $\begin{array}{l}55 \\
52 \\
62 \\
48 \\
66\end{array}$ & $\begin{array}{l}28.7 \\
27.1 \\
25.8 \\
25.1 \\
23.2\end{array}$ & $\begin{array}{l}33.1 \\
30.3 \\
29.4 \\
27.8 \\
26.4\end{array}$ & $\begin{array}{l}49.1 \\
43.1 \\
43.4 \\
41.3 \\
37.3\end{array}$ & $\begin{array}{l}19.5 \\
18.7 \\
14.6 \\
13.3 \\
14.5\end{array}$ \\
\hline $\begin{array}{c}6 \ldots \ldots \ldots \\
7 \ldots \ldots \ldots \\
8 \ldots \ldots \ldots \\
9 \ldots \ldots \ldots \\
10 \ldots \ldots\end{array}$ & $\begin{array}{l}-- \\
-- \\
-- \\
-- \\
--\end{array}$ & $\begin{array}{l}401 \\
396 \\
403 \\
408 \\
414\end{array}$ & $\begin{array}{l}352 \\
351 \\
337 \\
356 \\
351\end{array}$ & $\begin{array}{l}88 \\
88 \\
85 \\
89 \\
85\end{array}$ & $\begin{array}{l}62 \\
68 \\
59 \\
53 \\
55\end{array}$ & $\begin{array}{l}20.3 \\
20.7 \\
22.6 \\
22.6 \\
22.4\end{array}$ & $\begin{array}{l}24.0 \\
23.7 \\
25.7 \\
26.6 \\
27.0\end{array}$ & $\begin{array}{l}39.0 \\
36.0 \\
37.4 \\
42.9 \\
42.9\end{array}$ & $\begin{array}{r}8.3 \\
9.1 \\
16.8 \\
10.7 \\
10.7\end{array}$ \\
\hline $\begin{array}{l}11 \ldots \ldots \ldots \\
12 \ldots \ldots \\
13 \ldots \ldots \\
14 \ldots \ldots \ldots \\
15 \ldots \ldots \ldots\end{array}$ & $\begin{array}{l}-- \\
-- \\
-- \\
--\end{array}$ & $\begin{array}{l}418 \\
412 \\
419 \\
409 \\
404\end{array}$ & $\begin{array}{l}350 \\
350 \\
352 \\
330 \\
308\end{array}$ & $\begin{array}{l}85 \\
87 \\
87 \\
83 \\
77\end{array}$ & $\begin{array}{l}59 \\
60 \\
57 \\
54 \\
46\end{array}$ & $\begin{array}{l}22.9 \\
22.9 \\
22.9 \\
23.4 \\
22.4\end{array}$ & $\begin{array}{l}27.0 \\
26.3 \\
27.1 \\
26.3 \\
25.6\end{array}$ & $\begin{array}{l}44.2 \\
41.2 \\
45.2 \\
40.1 \\
40.3\end{array}$ & $\begin{array}{r}8.9 \\
10.0 \\
8.8 \\
9.3 \\
9.8\end{array}$ \\
\hline
\end{tabular}


Table 1. Radiation and temperature field data for undisturbed-land-surface site, May 1985 through June 1986 -Continued

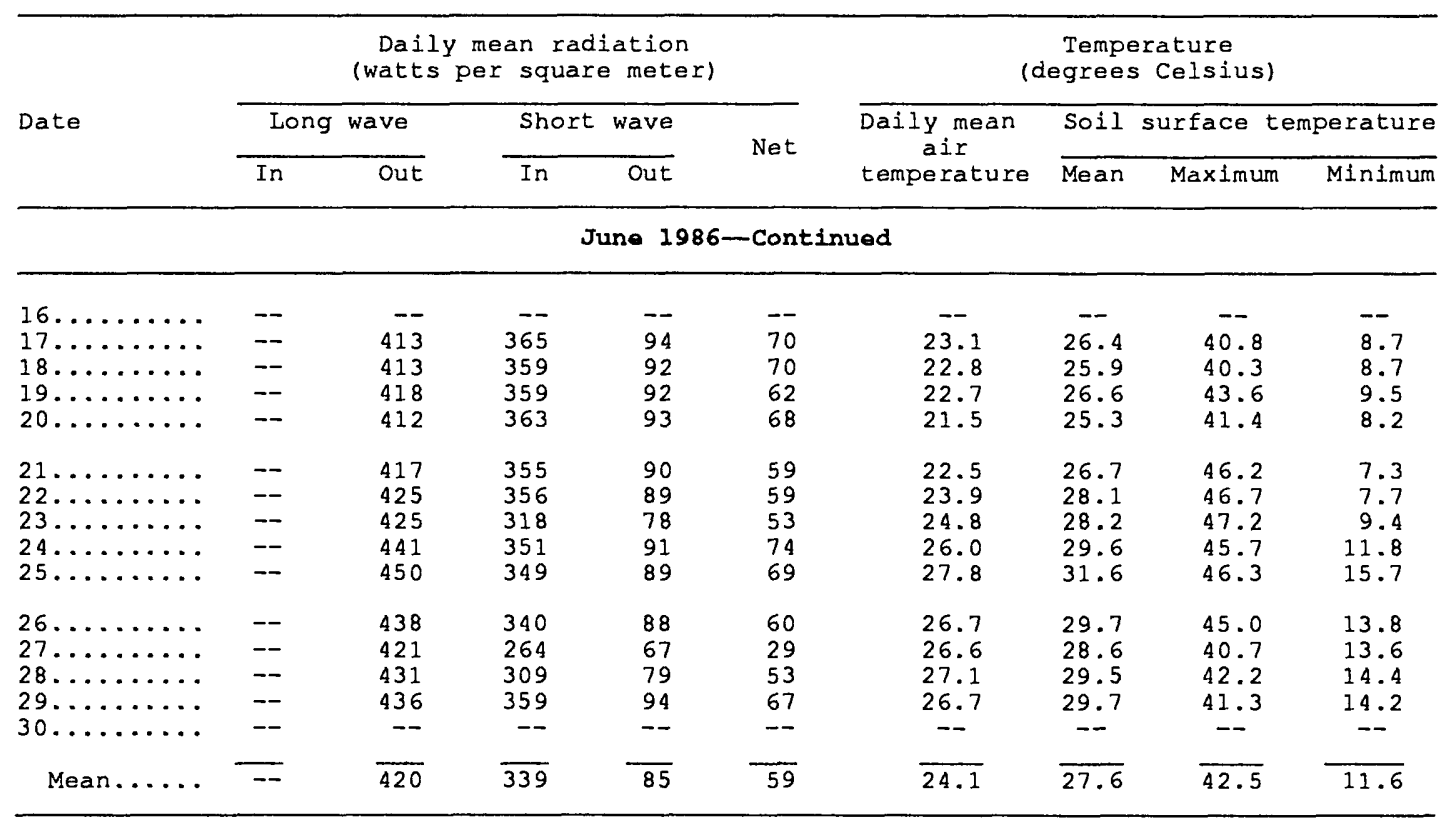


Table 2. Radiation and temperature field data for crater 1, May 1985 through June 1986

[Daily mean radiation: Pyranometer measured short-wave radiation; pyrgeometer measured long-wave radiation; net radiometer measured net radiation. In, incoming radiation; Out, outgoing radiation. Daily mean air temperature: Values were obtained from thermistor. - , no data or not calculated]

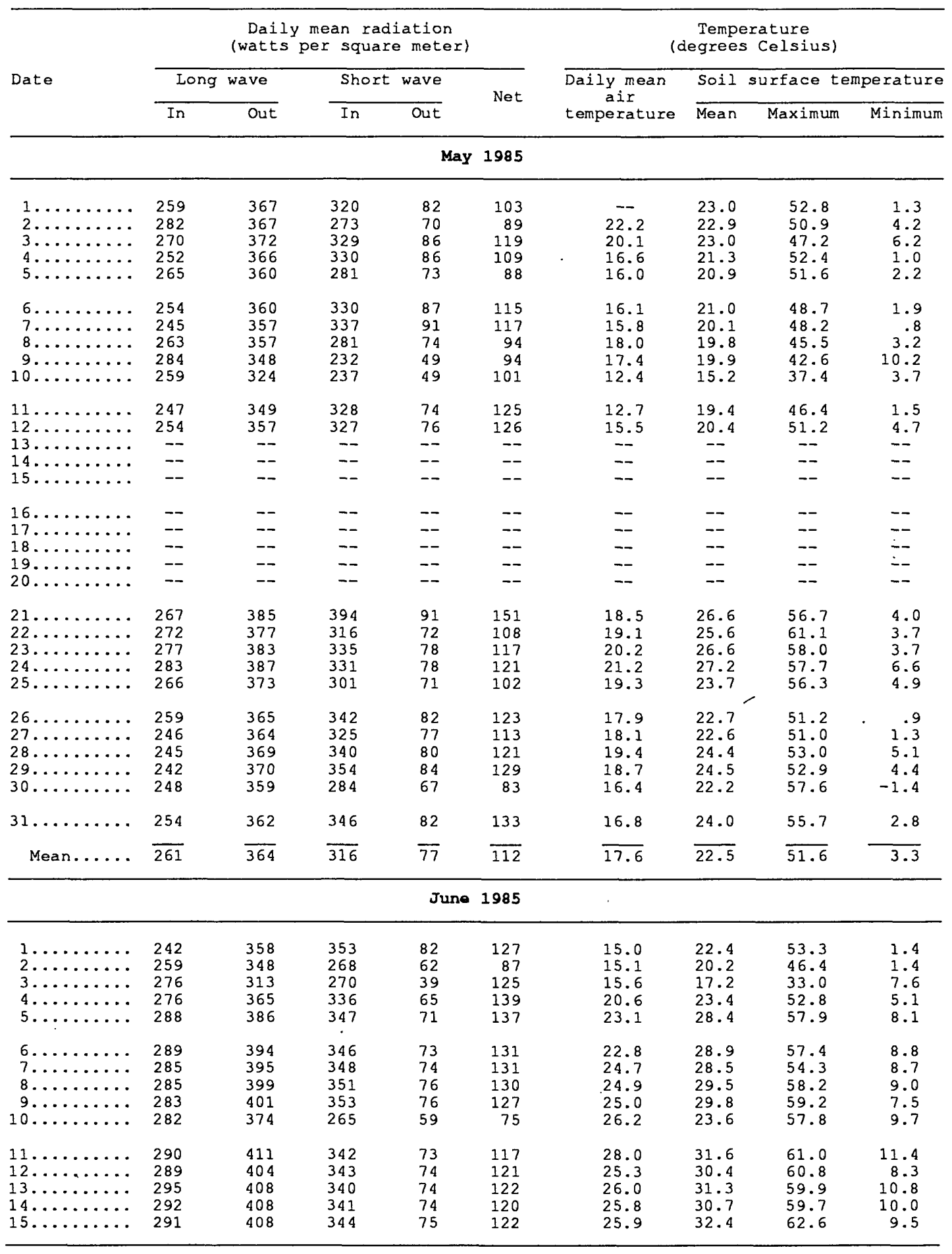


Table 2. Radiation and temperature field data for crater 1, May 1985 through June 1986 Continued

\begin{tabular}{|c|c|c|c|c|c|c|c|c|c|}
\hline \multirow{3}{*}{ Date } & \multicolumn{5}{|c|}{$\begin{array}{l}\text { Daily mean radiation } \\
\text { (watts per square meter) }\end{array}$} & \multicolumn{4}{|c|}{$\begin{array}{c}\text { Temperature } \\
\text { (degrees Celsius) }\end{array}$} \\
\hline & \multicolumn{2}{|c|}{ Long wave } & \multicolumn{2}{|c|}{ Short wave } & \multirow{2}{*}{ Net } & \multirow{2}{*}{$\begin{array}{c}\text { Daily mean } \\
\text { air } \\
\text { temperature }\end{array}$} & \multicolumn{2}{|c|}{ Soil surface $t$} & temperature \\
\hline & In & Out & In & $\overline{\text { Out }}$ & & & Mean & Maximum & Minimum \\
\hline \multicolumn{10}{|c|}{ June 1985 -Continued } \\
\hline $\begin{array}{l}16 \ldots \ldots \ldots \\
17 \ldots \ldots \ldots \\
18 \ldots \ldots \ldots \\
19 \ldots \ldots \ldots \\
20 \ldots \ldots \ldots\end{array}$ & $\begin{array}{l}284 \\
289 \\
306 \\
305 \\
280\end{array}$ & $\begin{array}{l}406 \\
410 \\
414 \\
417 \\
404\end{array}$ & $\begin{array}{l}350 \\
347 \\
312 \\
346 \\
351\end{array}$ & $\begin{array}{l}77 \\
76 \\
67 \\
77 \\
79\end{array}$ & $\begin{array}{r}124 \\
119 \\
104 \\
-- \\
--\end{array}$ & $\begin{array}{l}26.4 \\
27.4 \\
28.2 \\
27.5 \\
24.3\end{array}$ & $\begin{array}{l}31.8 \\
32.6 \\
33.3 \\
33.4 \\
30.7\end{array}$ & $\begin{array}{l}63.3 \\
65.3 \\
66.7 \\
62.8 \\
61.0\end{array}$ & $\begin{array}{r}8.1 \\
8.0 \\
10.2 \\
10.2 \\
9.7\end{array}$ \\
\hline $\begin{array}{l}21 \ldots \ldots \ldots \\
22 \ldots \ldots \ldots \\
23 \ldots \ldots \ldots \\
24 \ldots \ldots \ldots \\
25 \ldots \ldots \ldots \ldots\end{array}$ & $\begin{array}{l}263 \\
276 \\
285 \\
300 \\
254\end{array}$ & $\begin{array}{l}396 \\
399 \\
387 \\
375 \\
378\end{array}$ & $\begin{array}{l}353 \\
350 \\
298 \\
227 \\
362\end{array}$ & $\begin{array}{l}79 \\
79 \\
65 \\
45 \\
80\end{array}$ & $\begin{array}{l}-- \\
-- \\
-- \\
\overline{121}\end{array}$ & $\begin{array}{l}23.2 \\
24.3 \\
24.1 \\
21.8 \\
19.8\end{array}$ & $\begin{array}{l}29.6 \\
29.7 \\
27.6 \\
25.9 \\
27.3\end{array}$ & $\begin{array}{l}63.3 \\
60.4 \\
60.6 \\
56.4 \\
58.6\end{array}$ & $\begin{array}{r}4.7 \\
7.1 \\
6.8 \\
12.0 \\
6.2\end{array}$ \\
\hline $\begin{array}{l}26 \ldots \ldots \ldots \\
27 \ldots \ldots \ldots \\
28 \ldots \ldots \ldots \\
29 \ldots \ldots \ldots \\
30 \ldots \ldots \ldots\end{array}$ & $\begin{array}{l}242 \\
255 \\
272 \\
290 \\
253\end{array}$ & $\begin{array}{l}378 \\
387 \\
383 \\
372 \\
381\end{array}$ & $\begin{array}{l}362 \\
360 \\
308 \\
205 \\
361\end{array}$ & $\begin{array}{l}79 \\
79 \\
68 \\
43 \\
80\end{array}$ & $\begin{array}{r}101 \\
103 \\
84 \\
41 \\
105\end{array}$ & $\begin{array}{l}21.6 \\
21.6 \\
24.3 \\
20.8 \\
25.5\end{array}$ & $\begin{array}{l}27.1 \\
28.2 \\
26.1 \\
23.7 \\
24.6\end{array}$ & $\begin{array}{l}61.1 \\
63.0 \\
59.1 \\
47.0 \\
53.0\end{array}$ & $\begin{array}{l}2.0 \\
2.4 \\
3.5 \\
9.4 \\
3.1\end{array}$ \\
\hline Mean..... & $\overline{279}$ & $\overline{389}$ & $\overline{328}$ & $\overline{71}$ & $\overline{113}$ & $\overline{23.5}$ & $\overline{28.0}$ & $\overline{57.9}$ & 7.4 \\
\hline \multicolumn{10}{|c|}{ July 1985} \\
\hline $\begin{array}{l}1 \ldots \ldots \\
2 \ldots \ldots \\
3 \ldots \ldots \\
4 \ldots \ldots \\
5 \ldots \ldots\end{array}$ & $\begin{array}{l}271 \\
274 \\
287 \\
299 \\
305\end{array}$ & $\begin{array}{l}394 \\
401 \\
409 \\
419 \\
419\end{array}$ & $\begin{array}{l}351 \\
354 \\
350 \\
346 \\
329\end{array}$ & $\begin{array}{l}78 \\
78 \\
78 \\
78 \\
73\end{array}$ & $\begin{array}{r}102 \\
101 \\
100 \\
102 \\
94\end{array}$ & $\begin{array}{l}-- \\
-- \\
-- \\
-- \\
--\end{array}$ & $\begin{array}{l}27.5 \\
29.2 \\
31.0 \\
32.3 \\
32.6\end{array}$ & $\begin{array}{l}57.7 \\
59.5 \\
60.3 \\
58.7 \\
60.2\end{array}$ & $\begin{array}{r}5.1 \\
5.7 \\
6.7 \\
9.6 \\
11.2\end{array}$ \\
\hline $\begin{array}{c}6 \ldots \ldots \ldots \\
7 \ldots \ldots \\
8 \ldots \ldots \\
9 \ldots \ldots \\
10 \ldots \ldots\end{array}$ & $\begin{array}{l}331 \\
328 \\
325 \\
35.5 \\
349\end{array}$ & $\begin{array}{l}425 \\
406 \\
412 \\
436 \\
431\end{array}$ & $\begin{array}{l}285 \\
295 \\
289 \\
305 \\
296\end{array}$ & $\begin{array}{l}63 \\
60 \\
59 \\
65 \\
63\end{array}$ & $\begin{array}{r}82 \\
97 \\
92 \\
108 \\
102\end{array}$ & $\begin{array}{l}-- \\
-- \\
-- \\
-- \\
--\end{array}$ & $\begin{array}{l}33.6 \\
29.8 \\
31.0 \\
35.6 \\
35.6\end{array}$ & $\begin{array}{l}56.8 \\
58.6 \\
59.5 \\
60.2 \\
58.8\end{array}$ & $\begin{array}{l}16.7 \\
13.9 \\
11.4 \\
20.0 \\
19.2\end{array}$ \\
\hline $\begin{array}{l}11 \ldots \ldots \ldots \\
12 \ldots \ldots \\
13 \ldots \ldots \\
14 \ldots \ldots \\
15 \ldots \ldots\end{array}$ & $\begin{array}{l}332 \\
326 \\
309 \\
309 \\
320\end{array}$ & $\begin{array}{l}404 \\
384 \\
394 \\
389 \\
382\end{array}$ & $\begin{array}{l}325 \\
316 \\
334 \\
309 \\
296\end{array}$ & $\begin{array}{l}71 \\
70 \\
74 \\
69 \\
66\end{array}$ & $\begin{array}{r}108 \\
103 \\
106 \\
97 \\
93\end{array}$ & $\begin{array}{l}-- \\
-- \\
-- \\
-- \\
--\end{array}$ & $\begin{array}{l}32.7 \\
33.9 \\
32.2 \\
30.9 \\
31.0\end{array}$ & $\begin{array}{l}55.8 \\
58.0 \\
58.6 \\
57.2 \\
54.8\end{array}$ & $\begin{array}{l}14.8 \\
16.0 \\
11.4 \\
11.8 \\
11.2\end{array}$ \\
\hline $\begin{array}{l}16 \ldots \ldots \\
17 \ldots \ldots \\
18 \ldots \ldots \\
19 \ldots \ldots \\
20 \ldots \ldots\end{array}$ & $\begin{array}{l}330 \\
336 \\
338 \\
330 \\
333\end{array}$ & $\begin{array}{l}-- \\
-- \\
-- \\
-- \\
--\end{array}$ & $\begin{array}{l}199 \\
309 \\
187 \\
233 \\
243\end{array}$ & $\begin{array}{l}41 \\
68 \\
30 \\
45 \\
34\end{array}$ & $\begin{array}{r}47 \\
109 \\
76 \\
83 \\
123\end{array}$ & $\begin{array}{l}-- \\
-- \\
-- \\
-- \\
--\end{array}$ & $\begin{array}{l}28.2 \\
32.3 \\
25.2 \\
25.7 \\
23.3\end{array}$ & $\begin{array}{l}51.9 \\
56.2 \\
38.3 \\
53.9 \\
37.7\end{array}$ & $\begin{array}{l}13.6 \\
13.5 \\
17.7 \\
14.4 \\
16.5\end{array}$ \\
\hline $\begin{array}{l}21 \ldots \ldots \ldots \\
22 \ldots \ldots \ldots \\
23 \ldots \ldots \\
24 \ldots \ldots \\
25 \ldots \ldots \ldots\end{array}$ & $\begin{array}{l}325 \\
304 \\
320 \\
317 \\
320\end{array}$ & $\begin{array}{l}-- \\
-- \\
-- \\
-- \\
--\end{array}$ & $\begin{array}{l}257 \\
328 \\
309 \\
312 \\
289\end{array}$ & $\begin{array}{l}45 \\
68 \\
64 \\
64 \\
61\end{array}$ & $\begin{array}{r}120 \\
131 \\
109 \\
104 \\
96\end{array}$ & $\begin{array}{l}-- \\
-- \\
-- \\
--\end{array}$ & $\begin{array}{l}24.9 \\
28.3 \\
30.4 \\
32.0 \\
30.8\end{array}$ & $\begin{array}{l}42.2 \\
53.2 \\
56.8 \\
60.5 \\
60.9\end{array}$ & $\begin{array}{l}15.5 \\
12.0 \\
11.7 \\
13.6 \\
14.8\end{array}$ \\
\hline $\begin{array}{l}26 \ldots \ldots \ldots \\
27 \ldots \ldots \ldots \\
28 \ldots \ldots \ldots \\
29 \ldots \ldots \ldots \\
30 \ldots \ldots\end{array}$ & $\begin{array}{l}314 \\
316 \\
306 \\
293 \\
275\end{array}$ & $\begin{array}{l}-- \\
-- \\
-- \\
-- \\
--\end{array}$ & $\begin{array}{r}296 \\
302 \\
324 \\
331 \\
--\end{array}$ & $\begin{array}{l}62 \\
64 \\
70 \\
74 \\
75\end{array}$ & $\begin{array}{l}100 \\
101 \\
115 \\
120 \\
108\end{array}$ & $\begin{array}{l}-- \\
-- \\
-- \\
--\end{array}$ & $\begin{array}{l}30.8 \\
31.2 \\
30.0 \\
28.1 \\
27.2\end{array}$ & $\begin{array}{l}60.4 \\
62.6 \\
58.8 \\
53.9 \\
56.0\end{array}$ & $\begin{array}{r}12.3 \\
12.1 \\
12.7 \\
10.0 \\
7.4\end{array}$ \\
\hline $31 \ldots \ldots \ldots$ & 272 & -- & 339 & 77 & 120 & -- & 25.6 & 52.8 & 5.1 \\
\hline Mean...... & $\overline{314}$ & $\overline{407}$ & $\overline{301}$ & $\overline{64}$ & $\overline{102}$ & -- & $\overline{30.1}$ & $\overline{55.8}$ & 12.5 \\
\hline
\end{tabular}


Table 2. Radiation and temperature field data for crater 1, May 1985 through June $1986-$ Continued

\begin{tabular}{|c|c|c|c|c|c|c|c|c|c|}
\hline \multirow{3}{*}{ Date } & \multicolumn{5}{|c|}{$\begin{array}{l}\text { Daily mean radiation } \\
\text { (watts per square meter) }\end{array}$} & \multicolumn{4}{|c|}{$\begin{array}{c}\text { Temperature } \\
\text { (degrees Celsius) }\end{array}$} \\
\hline & \multicolumn{2}{|c|}{ Long wave } & \multicolumn{2}{|c|}{ Short wave } & \multirow{2}{*}{ Net } & \multirow{2}{*}{$\begin{array}{c}\text { Daily mean } \\
\text { air } \\
\text { temperature }\end{array}$} & \multirow{2}{*}{ Soil } & \multirow{2}{*}{$\frac{\text { surface } t}{\text { Maximum }}$} & temperature \\
\hline & In & Out & In & out & & & & & Minimum \\
\hline \multicolumn{10}{|c|}{ August 1985} \\
\hline $\begin{array}{l}1 \ldots \ldots \\
2 \ldots \ldots \\
3 \ldots \ldots \\
4 \ldots \ldots \\
5 \ldots \ldots\end{array}$ & $\begin{array}{l}278 \\
273 \\
277 \\
281 \\
277\end{array}$ & $\begin{array}{l}-- \\
-- \\
-- \\
-- \\
--\end{array}$ & $\begin{array}{l}338 \\
333 \\
330 \\
331 \\
330\end{array}$ & $\begin{array}{l}77 \\
75 \\
73 \\
75 \\
75\end{array}$ & $\begin{array}{l}122 \\
108 \\
105 \\
108 \\
103\end{array}$ & $\begin{array}{l}-- \\
-- \\
-- \\
-- \\
--\end{array}$ & $\begin{array}{l}25.3 \\
26.7 \\
27.6 \\
27.9 \\
27.8\end{array}$ & $\begin{array}{l}51.7 \\
57.6 \\
60.0 \\
58.1 \\
60.0\end{array}$ & $\begin{array}{l}5.2 \\
5.4 \\
4.9 \\
5.4 \\
4.7\end{array}$ \\
\hline $\begin{array}{r}6 \ldots \ldots \ldots \\
7 \ldots \ldots \ldots \\
8 \ldots \ldots \ldots \\
9 \ldots \ldots \ldots \\
10 \ldots \ldots \ldots\end{array}$ & $\begin{array}{l}278 \\
280 \\
288 \\
287 \\
286\end{array}$ & $\begin{array}{l}-- \\
-- \\
-- \\
-- \\
--\end{array}$ & $\begin{array}{l}328 \\
311 \\
308 \\
323 \\
320\end{array}$ & $\begin{array}{l}75 \\
72 \\
72 \\
72 \\
73\end{array}$ & $\begin{array}{r}105 \\
98 \\
104 \\
103 \\
112\end{array}$ & $\begin{array}{l}-- \\
-- \\
-- \\
-- \\
--\end{array}$ & $\begin{array}{l}27.8 \\
26.8 \\
26.9 \\
28.9 \\
27.5\end{array}$ & $\begin{array}{l}60.7 \\
56.5 \\
57.3 \\
60.5 \\
56.2\end{array}$ & $\begin{array}{l}5.4 \\
5.6 \\
6.8 \\
7.0 \\
6.2\end{array}$ \\
\hline $\begin{array}{l}11 \ldots \ldots \ldots \\
12 \ldots \ldots \\
13 \ldots \ldots \ldots \\
14 \ldots \ldots \ldots \\
15 \ldots \ldots \ldots\end{array}$ & $\begin{array}{r}282 \\
-- \\
-- \\
-- \\
--\end{array}$ & $\begin{array}{l}-- \\
-- \\
-- \\
-- \\
--\end{array}$ & $\begin{array}{l}310 \\
315 \\
312 \\
284 \\
302\end{array}$ & $\begin{array}{l}69 \\
71 \\
72 \\
64 \\
70\end{array}$ & $\begin{array}{l}99 \\
96 \\
99 \\
83 \\
97\end{array}$ & $\begin{array}{l}-- \\
-- \\
-- \\
-- \\
--\end{array}$ & $\begin{array}{l}28.8 \\
28.3 \\
26.9 \\
26.4 \\
27.8\end{array}$ & $\begin{array}{l}60.6 \\
58.8 \\
59.2 \\
60.5 \\
59.4\end{array}$ & $\begin{array}{l}7.0 \\
8.6 \\
4.8 \\
4.8 \\
6.6\end{array}$ \\
\hline $\begin{array}{l}16 \ldots \ldots \ldots \\
17 \ldots \ldots \\
18 \ldots \ldots \ldots \\
19 \ldots \ldots \\
20 \ldots \ldots \ldots\end{array}$ & $\begin{array}{l}-- \\
-- \\
-- \\
--\end{array}$ & $\begin{array}{l}-- \\
-- \\
-- \\
-- \\
--\end{array}$ & $\begin{array}{l}283 \\
255 \\
292 \\
261 \\
315\end{array}$ & $\begin{array}{l}66 \\
62 \\
75 \\
62 \\
74\end{array}$ & $\begin{array}{r}98 \\
94 \\
79 \\
69 \\
106\end{array}$ & $\begin{array}{l}-- \\
-- \\
-- \\
-- \\
--\end{array}$ & $\begin{array}{l}27.7 \\
26.7 \\
24.7 \\
22.4 \\
25.8\end{array}$ & $\begin{array}{l}59.3 \\
56.0 \\
53.3 \\
68.0 \\
55.4\end{array}$ & $\begin{array}{l}7.6 \\
8.8 \\
4.9 \\
3.4 \\
5.8\end{array}$ \\
\hline $\begin{array}{l}21 \ldots \ldots \ldots \\
22 \ldots \ldots \ldots \\
23 \ldots \ldots \ldots \\
24 \ldots \ldots \ldots \\
25 \ldots \ldots \ldots\end{array}$ & $\begin{array}{l}-- \\
-- \\
-- \\
--\end{array}$ & $\begin{array}{l}-- \\
-- \\
-- \\
-- \\
--\end{array}$ & $\begin{array}{l}278 \\
289 \\
295 \\
294 \\
288\end{array}$ & $\begin{array}{l}64 \\
65 \\
65 \\
65 \\
66\end{array}$ & $\begin{array}{l}84 \\
91 \\
95 \\
93 \\
96\end{array}$ & $\begin{array}{l}-- \\
-- \\
-- \\
-- \\
--\end{array}$ & $\begin{array}{l}24.4 \\
25.9 \\
28.4 \\
29.5 \\
30.0\end{array}$ & $\begin{array}{l}55.3 \\
57.6 \\
62.2 \\
62.5 \\
61.2\end{array}$ & $\begin{array}{l}5.7 \\
5.1 \\
5.8 \\
6.9 \\
9.2\end{array}$ \\
\hline $\begin{array}{l}26 \ldots \ldots \ldots \\
27 \ldots \ldots \ldots \\
28 \ldots \ldots \ldots \\
29 \ldots \ldots \\
30 \ldots \ldots \ldots\end{array}$ & $\begin{array}{l}-- \\
-- \\
-- \\
--\end{array}$ & $\begin{array}{l}-- \\
-- \\
-- \\
-- \\
--\end{array}$ & $\begin{array}{l}281 \\
291 \\
296 \\
289 \\
292\end{array}$ & $\begin{array}{l}64 \\
66 \\
68 \\
66 \\
63\end{array}$ & $\begin{array}{l}98 \\
99 \\
95 \\
73 \\
94\end{array}$ & $\begin{array}{l}-- \\
-- \\
-- \\
-- \\
--\end{array}$ & $\begin{array}{l}30.6 \\
29.2 \\
26.8 \\
27.0 \\
28.1\end{array}$ & $\begin{array}{l}58.8 \\
58.2 \\
58.2 \\
59.5 \\
58.5\end{array}$ & $\begin{array}{r}12.5 \\
11.2 \\
7.0 \\
5.7 \\
7.5\end{array}$ \\
\hline $31 \ldots \ldots \ldots$ & -- & -- & 284 & 65 & 93 & -- & 28.9 & 59.4 & 9.0 \\
\hline Mean..... & $\overline{281}$ & -- & $\overline{302}$ & $\overline{69}$ & 97 & - & $\overline{27.4}$ & $\overline{58.7}$ & 6.6 \\
\hline \multicolumn{10}{|c|}{ September 1985} \\
\hline $\begin{array}{l}1 \ldots \ldots \\
2 \ldots \ldots \\
3 \ldots \ldots \\
4 \ldots \ldots \\
5 \ldots \ldots\end{array}$ & $\begin{array}{l}-- \\
-- \\
-- \\
--\end{array}$ & $\begin{array}{l}-- \\
-- \\
-- \\
-- \\
--\end{array}$ & $\begin{array}{l}275 \\
284 \\
285 \\
227 \\
196\end{array}$ & $\begin{array}{l}65 \\
66 \\
67 \\
50 \\
42\end{array}$ & $\begin{array}{l}98 \\
93 \\
95 \\
65 \\
48\end{array}$ & $\begin{array}{l}-- \\
-- \\
-- \\
-- \\
--\end{array}$ & $\begin{array}{l}27.9 \\
27.0 \\
24.9 \\
20.8 \\
19.9\end{array}$ & $\begin{array}{l}54.0 \\
54.3 \\
54.0 \\
51.1 \\
54.3\end{array}$ & $\begin{array}{r}10.3 \\
10.2 \\
5.9 \\
3.5 \\
4.8\end{array}$ \\
\hline $\begin{array}{r}6 \ldots \ldots \ldots \\
7 \ldots \ldots \ldots \\
8 \ldots \ldots \ldots \\
9 \ldots \ldots \ldots \\
10 \ldots \ldots \ldots\end{array}$ & $\begin{array}{l}-- \\
-- \\
-- \\
--\end{array}$ & $\begin{array}{l}-- \\
-- \\
-- \\
--\end{array}$ & $\begin{array}{l}193 \\
257 \\
237 \\
262 \\
272\end{array}$ & $\begin{array}{l}41 \\
58 \\
52 \\
64 \\
63\end{array}$ & $\begin{array}{l}51 \\
87 \\
84 \\
94 \\
90\end{array}$ & $\begin{array}{l}-- \\
-- \\
-- \\
-- \\
--\end{array}$ & $\begin{array}{l}17.5 \\
20.4 \\
21.1 \\
20.1 \\
18.6\end{array}$ & $\begin{array}{l}51.6 \\
49.4 \\
48.6 \\
44.7 \\
48.1\end{array}$ & $\begin{array}{r}2.8 \\
1.5 \\
5.7 \\
6.5 \\
.7\end{array}$ \\
\hline $\begin{array}{l}11 \ldots \ldots \ldots \\
12 \ldots \ldots \\
13 \ldots \ldots \\
14 \ldots \ldots \\
15 \ldots \ldots\end{array}$ & $\begin{array}{l}-- \\
-- \\
-- \\
-- \\
--\end{array}$ & $\begin{array}{l}-- \\
-- \\
-- \\
-- \\
--\end{array}$ & $\begin{array}{l}196 \\
265 \\
250 \\
212 \\
254\end{array}$ & $\begin{array}{l}46 \\
61 \\
59 \\
60 \\
59\end{array}$ & $\begin{array}{l}55 \\
83 \\
82 \\
81 \\
83\end{array}$ & $\begin{array}{l}-- \\
-- \\
-- \\
-- \\
--\end{array}$ & $\begin{array}{l}14.9 \\
17.7 \\
19.6 \\
20.1 \\
20.9\end{array}$ & $\begin{array}{l}45.8 \\
51.1 \\
51.3 \\
49.4 \\
51.0\end{array}$ & $\begin{array}{r}2.1 \\
-3.2 \\
-.2 \\
1.2 \\
2.9\end{array}$ \\
\hline
\end{tabular}


Table 2. Radiation and temperature field data for crater 1, May 1985 through June $1986-$ Continued

\begin{tabular}{|c|c|c|c|c|c|c|c|c|c|}
\hline \multirow{3}{*}{ Date } & \multicolumn{5}{|c|}{$\begin{array}{l}\text { Daily mean radiation } \\
\text { (watts per square meter) }\end{array}$} & \multicolumn{4}{|c|}{$\begin{array}{l}\text { Temperature } \\
\text { (degrees Celsius) }\end{array}$} \\
\hline & \multicolumn{2}{|c|}{ Long wave } & \multicolumn{2}{|c|}{ Short wave } & \multirow{2}{*}{ Net } & \multirow{2}{*}{$\begin{array}{c}\text { Daily mean } \\
\text { air } \\
\text { temperature }\end{array}$} & \multirow{2}{*}{ Soil } & \multirow{2}{*}{$\begin{array}{r}\text { surface te } \\
\text { Maximum }\end{array}$} & temperature \\
\hline & In & out & In & Out & & & & & Minimum \\
\hline \multicolumn{10}{|c|}{ September 1985-Continued } \\
\hline $\begin{array}{l}16 \ldots \ldots \ldots \\
17 \ldots \ldots \ldots \\
18 \ldots \ldots \ldots \\
19 \ldots \ldots \ldots \\
20 \ldots \ldots \ldots\end{array}$ & $\begin{array}{l}-- \\
276 \\
312 \\
287 \\
250\end{array}$ & $\begin{array}{l}-- \\
-- \\
-- \\
-- \\
--\end{array}$ & $\begin{array}{r}254 \\
253 \\
68 \\
176 \\
214\end{array}$ & $\begin{array}{r}61 \\
61 \\
9 \\
31 \\
52\end{array}$ & $\begin{array}{l}81 \\
89 \\
-3 \\
62 \\
93\end{array}$ & $\begin{array}{l}-- \\
-- \\
-- \\
--\end{array}$ & $\begin{array}{l}19.8 \\
19.4 \\
15.8 \\
15.4 \\
16.5\end{array}$ & $\begin{array}{l}49.4 \\
46.5 \\
27.9 \\
33.6 \\
46.2\end{array}$ & $\begin{array}{l}0.8 \\
1.0 \\
9.2 \\
4.9 \\
-.5\end{array}$ \\
\hline $\begin{array}{l}21 \ldots \ldots \ldots \ldots \\
22 \ldots \ldots \ldots \\
23 \ldots \ldots \ldots \\
24 \ldots \ldots \ldots \\
25 \ldots \ldots \ldots\end{array}$ & $\begin{array}{l}247 \\
262 \\
256 \\
270 \\
268\end{array}$ & $\begin{array}{l}-- \\
-- \\
-- \\
374 \\
374\end{array}$ & $\begin{array}{l}-- \\
-- \\
-- \\
-- \\
--\end{array}$ & $\begin{array}{l}54 \\
54 \\
52 \\
53 \\
52\end{array}$ & $\begin{array}{l}81 \\
84 \\
71 \\
73 \\
73\end{array}$ & $\begin{array}{l}-- \\
-- \\
-- \\
-- \\
--\end{array}$ & $\begin{array}{l}16.7 \\
18.6 \\
19.2 \\
20.8 \\
21.4\end{array}$ & $\begin{array}{l}46.2 \\
48.3 \\
50.9 \\
52.1 \\
53.6\end{array}$ & $\begin{array}{r}-2.2 \\
.9 \\
-.2 \\
1.1 \\
2.1\end{array}$ \\
\hline $\begin{array}{l}26 \ldots \ldots \ldots \ldots \\
27 \ldots \ldots \ldots \\
28 \ldots \ldots \ldots \\
29 \ldots \ldots \ldots \\
30 \ldots \ldots \ldots\end{array}$ & $\begin{array}{l}266 \\
295 \\
266 \\
240 \\
266\end{array}$ & $\begin{array}{l}365 \\
360 \\
362 \\
342 \\
352\end{array}$ & $\begin{array}{l}-- \\
-- \\
-- \\
-- \\
--\end{array}$ & $\begin{array}{l}52 \\
33 \\
47 \\
49 \\
54\end{array}$ & $\begin{array}{l}69 \\
42 \\
77 \\
60 \\
64\end{array}$ & $\begin{array}{l}-- \\
-- \\
-- \\
--\end{array}$ & $\begin{array}{l}20.5 \\
19.3 \\
19.7 \\
16.4 \\
18.5\end{array}$ & $\begin{array}{l}50.8 \\
48.3 \\
49.9 \\
47.8 \\
47.4\end{array}$ & $\begin{array}{r}1.6 \\
5.5 \\
3.5 \\
-3.5 \\
1.2\end{array}$ \\
\hline Mean..... & $\overline{269}$ & $\overline{361}$ & $\overline{232}$ & $\overline{52}$ & $\overline{74}$ & -- & $\overline{19.6}$ & $\overline{48.6}$ & 2.7 \\
\hline \multicolumn{10}{|c|}{ October 1985} \\
\hline $\begin{array}{l}1 \ldots \ldots \\
2 \ldots \ldots \\
3 \ldots \ldots \\
4 \ldots \ldots \\
5 \ldots \ldots\end{array}$ & $\begin{array}{l}271 \\
252 \\
266 \\
272 \\
263\end{array}$ & $\begin{array}{l}357 \\
349 \\
360 \\
367 \\
355\end{array}$ & $\begin{array}{l}-- \\
-- \\
-- \\
-- \\
--\end{array}$ & $\begin{array}{l}50 \\
54 \\
54 \\
52 \\
54\end{array}$ & $\begin{array}{l}55 \\
64 \\
67 \\
56 \\
61\end{array}$ & $\begin{array}{l}-- \\
-- \\
-- \\
-- \\
--\end{array}$ & $\begin{array}{l}19.8 \\
18.0 \\
19.8 \\
20.6 \\
19.1\end{array}$ & $\begin{array}{l}54.3 \\
48.9 \\
51.1 \\
50.7 \\
49.6\end{array}$ & $\begin{array}{r}2.7 \\
-1.5 \\
.9 \\
3.8 \\
.4\end{array}$ \\
\hline $\begin{array}{c}6 \ldots \ldots \ldots \\
7 \ldots \ldots \\
8 \ldots \ldots \ldots \\
9 \ldots \ldots \ldots \\
10 \ldots \ldots\end{array}$ & $\begin{array}{l}294 \\
277 \\
271 \\
267 \\
261\end{array}$ & $\begin{array}{l}362 \\
348 \\
306 \\
294 \\
306\end{array}$ & $\begin{array}{l}-- \\
-- \\
-- \\
-- \\
--\end{array}$ & $\begin{array}{r}52 \\
53 \\
8 \\
18 \\
23\end{array}$ & $\begin{array}{l}68 \\
65 \\
10 \\
34 \\
36\end{array}$ & $\begin{array}{l}-- \\
-- \\
-- \\
-- \\
--\end{array}$ & $\begin{array}{r}20.5 \\
18.8 \\
10.7 \\
7.6 \\
8.7\end{array}$ & $\begin{array}{l}47.7 \\
45.0 \\
22.6 \\
26.3 \\
27.3\end{array}$ & $\begin{array}{r}6.8 \\
2.2 \\
1.3 \\
-1.1 \\
.6\end{array}$ \\
\hline $\begin{array}{l}11 \ldots \ldots \\
12 \ldots \ldots \\
13 \ldots \ldots \\
14 \ldots \ldots \\
15 \ldots \ldots\end{array}$ & $\begin{array}{l}237 \\
239 \\
233 \\
211 \\
219\end{array}$ & $\begin{array}{l}310 \\
314 \\
321 \\
313 \\
316\end{array}$ & $\begin{array}{l}-- \\
-- \\
-- \\
-- \\
--\end{array}$ & $\begin{array}{l}46 \\
42 \\
47 \\
50 \\
49\end{array}$ & $\begin{array}{l}73 \\
49 \\
61 \\
-- \\
--\end{array}$ & $\begin{array}{l}-- \\
-- \\
-- \\
-- \\
--\end{array}$ & $\begin{array}{l}10.3 \\
11.6 \\
12.9 \\
10.3 \\
10.8\end{array}$ & $\begin{array}{l}35.0 \\
36.5 \\
39.0 \\
37.6 \\
38.8\end{array}$ & $\begin{array}{r}-3.7 \\
-4.1 \\
.3 \\
-4.6 \\
-4.9\end{array}$ \\
\hline $\begin{array}{l}16 \ldots \ldots \ldots \\
17 \ldots \ldots \\
18 \ldots \ldots \\
19 \ldots \ldots \\
20 \ldots \ldots\end{array}$ & $\begin{array}{l}222 \\
230 \\
230 \\
236 \\
254\end{array}$ & $\begin{array}{l}313 \\
320 \\
320 \\
317 \\
322\end{array}$ & $\begin{array}{l}-- \\
-- \\
-- \\
--\end{array}$ & $\begin{array}{l}47 \\
45 \\
45 \\
44 \\
34\end{array}$ & $\begin{array}{l}-- \\
-- \\
-- \\
--\end{array}$ & $\begin{array}{l}-- \\
-- \\
-- \\
--\end{array}$ & $\begin{array}{l}11 \cdot 3 \\
12 \cdot 3 \\
12 \cdot 4 \\
12 \cdot 3 \\
12 \cdot 6\end{array}$ & $\begin{array}{l}41.8 \\
43.0 \\
41.3 \\
40.8 \\
39.0\end{array}$ & $\begin{array}{l}-6.0 \\
-5.5 \\
-4.6 \\
-4.0 \\
-1.0\end{array}$ \\
\hline $\begin{array}{l}21 \ldots \ldots \ldots \\
22 \ldots \ldots \ldots \\
23 \ldots \ldots \\
24 \ldots \ldots \ldots \\
25 \ldots \ldots \ldots\end{array}$ & $\begin{array}{l}-- \\
228 \\
245 \\
239\end{array}$ & $\begin{array}{l}-- \\
-- \\
305 \\
320 \\
320\end{array}$ & $\begin{array}{l}-- \\
-- \\
-- \\
-- \\
--\end{array}$ & $\begin{array}{l}-- \\
-- \\
42 \\
42\end{array}$ & $\begin{array}{l}-- \\
-- \\
-- \\
-- \\
--\end{array}$ & $\begin{array}{l}-- \\
-- \\
-- \\
-- \\
--\end{array}$ & $\begin{array}{c}-- \\
11.8 \\
14.7 \\
14.5\end{array}$ & $\begin{array}{l}-- \\
40.3 \\
44.5 \\
43.4\end{array}$ & $\begin{array}{l}-- \\
-5.1 \\
-5.3 \\
-1.9\end{array}$ \\
\hline $\begin{array}{l}26 \ldots \ldots \ldots \\
27 \ldots \ldots \ldots \\
28 \ldots \ldots \\
29 \ldots \ldots \ldots \\
30 \ldots \ldots\end{array}$ & $\begin{array}{l}237 \\
237 \\
260 \\
234 \\
244\end{array}$ & $\begin{array}{l}319 \\
316 \\
309 \\
316 \\
313\end{array}$ & $\begin{array}{l}-- \\
-- \\
-- \\
-- \\
--\end{array}$ & $\begin{array}{l}41 \\
40 \\
18 \\
43 \\
40\end{array}$ & $\begin{array}{l}-- \\
-- \\
-- \\
--\end{array}$ & $\begin{array}{l}-- \\
-- \\
-- \\
-- \\
--\end{array}$ & $\begin{array}{l}14.7 \\
14.3 \\
11.2 \\
11.4 \\
11.0\end{array}$ & $\begin{array}{l}46.2 \\
44.6 \\
41.7 \\
38.7 \\
38.7\end{array}$ & $\begin{array}{r}-2.5 \\
-2.6 \\
1.5 \\
-3.9 \\
-5.5\end{array}$ \\
\hline $31 \ldots \ldots \ldots$ & 241 & 319 & -- & 53 & -- & -- & 12.0 & 30.7 & -.6 \\
\hline Mean..... & $\overline{247}$ & $\overline{324}$ & $\overline{--}$ & $\overline{42}$ & $\overline{54}$ & - & $\overline{13.7}$ & $\overline{40.9}$ & -1.5 \\
\hline
\end{tabular}


Table 2. Radiation and temperature field data for crater 1, May 1985 through June $1986-$ Continued

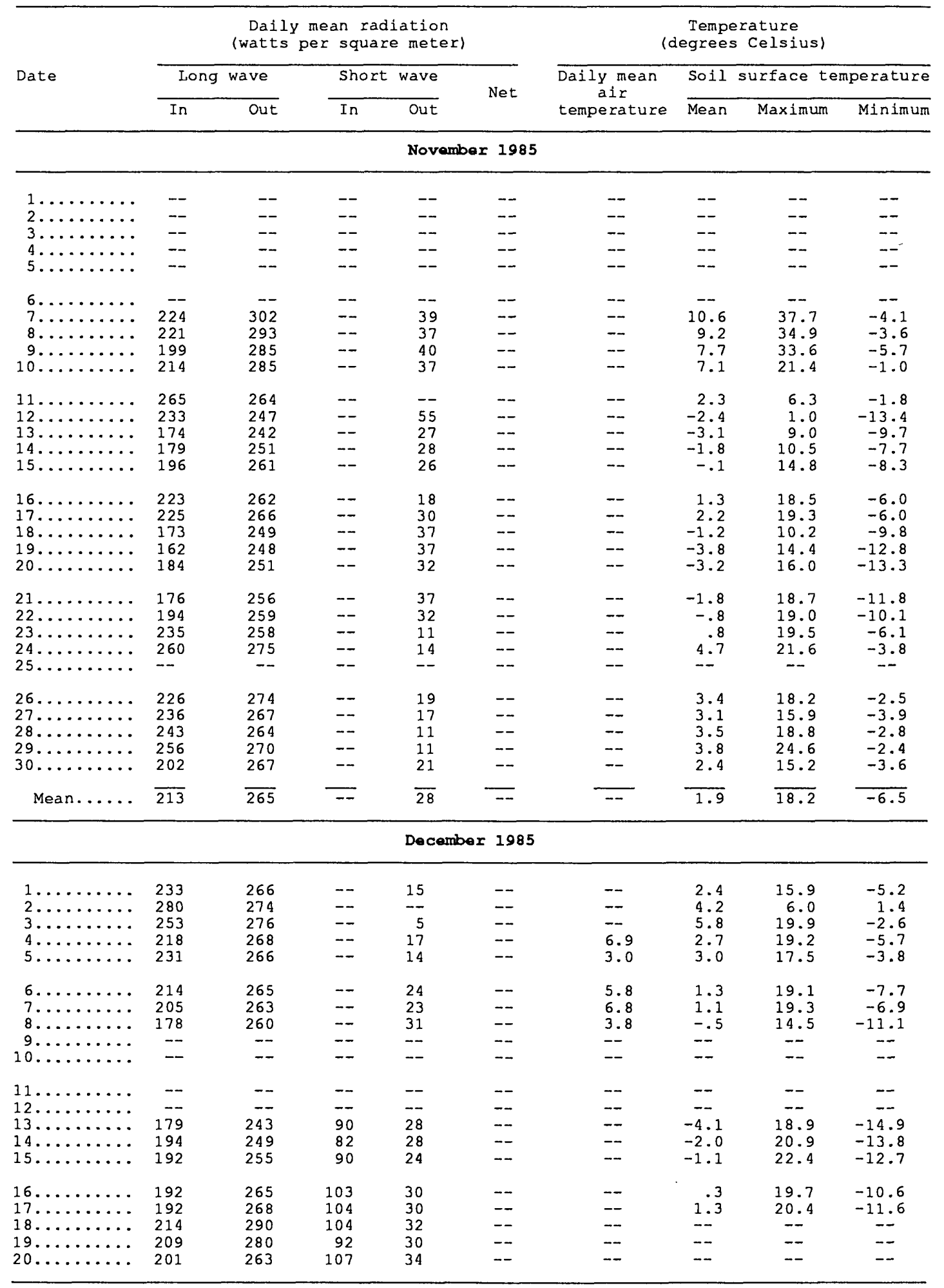


Table 2. Radiation and temperature field data for crater 1, May 1985 through June $1986-$ Continued

\begin{tabular}{|c|c|c|c|c|c|c|c|c|c|}
\hline \multirow{3}{*}{ Date } & \multicolumn{5}{|c|}{$\begin{array}{l}\text { Daily mean radiation } \\
\text { (watts per square meter) }\end{array}$} & \multicolumn{4}{|c|}{$\begin{array}{c}\text { Temperature } \\
\text { (degrees Celsius) }\end{array}$} \\
\hline & \multicolumn{2}{|c|}{ Long wave } & \multicolumn{2}{|c|}{ Short wave } & \multirow{2}{*}{ Net } & \multirow{2}{*}{$\begin{array}{c}\text { Daily mean } \\
\text { air } \\
\text { temperature }\end{array}$} & \multirow{2}{*}{$\frac{\text { Soil }}{\text { Mean }}$} & \multirow{2}{*}{$\begin{array}{r}\text { surface } t \\
\text { Maximum }\end{array}$} & temperature \\
\hline & In & Out & In & $\overline{\text { Out }}$ & & & & & Minimum \\
\hline \multicolumn{10}{|c|}{ December 1985-Continued } \\
\hline $\begin{array}{l}21 \ldots \ldots \cdots \\
22 \ldots \ldots \cdots \\
23 \ldots \ldots \cdots \\
24 \ldots \ldots \cdots \\
25 \ldots \ldots \cdots\end{array}$ & $\begin{array}{l}-- \\
203 \\
208 \\
207\end{array}$ & $\begin{array}{r}-- \\
272 \\
278 \\
280\end{array}$ & $\begin{array}{l}-- \\
\overline{86} \\
89 \\
96\end{array}$ & $\begin{array}{l}-- \\
-- \\
31 \\
29 \\
27\end{array}$ & $\begin{array}{l}-- \\
-- \\
-- \\
--\end{array}$ & $\begin{array}{l}-- \\
-- \\
-- \\
--\end{array}$ & $\begin{array}{l}=- \\
-- \\
4.0 \\
4.3\end{array}$ & $\begin{array}{c}-- \\
-- \\
-- \\
23.7 \\
26.7\end{array}$ & $\begin{array}{c}-- \\
-- \\
-- \\
-8.4 \\
-7.6\end{array}$ \\
\hline $\begin{array}{l}26 \ldots \ldots \ldots \\
27 \ldots \ldots \ldots \\
28 \ldots \ldots \cdots \\
29 \ldots \ldots \cdots \\
30 \ldots \ldots \\
30 \ldots \cdots\end{array}$ & $\begin{array}{l}196 \\
191 \\
194 \\
259 \\
261\end{array}$ & $\begin{array}{l}265 \\
261 \\
259 \\
268 \\
278\end{array}$ & $\begin{array}{l}99 \\
85 \\
85 \\
45 \\
44\end{array}$ & $\begin{array}{r}28 \\
29 \\
28 \\
7 \\
8\end{array}$ & $\begin{array}{l}-- \\
-- \\
-- \\
--\end{array}$ & $\begin{array}{l}-- \\
-- \\
-- \\
--\end{array}$ & $\begin{array}{r}.3 \\
-.4 \\
-.4 \\
4.1 \\
6.3\end{array}$ & $\begin{array}{l}25.7 \\
25.2 \\
24.2 \\
18.1 \\
26.7\end{array}$ & $\begin{array}{r}-11.4 \\
-12.5 \\
-11.9 \\
-7.4 \\
-3.1\end{array}$ \\
\hline $31 \ldots \ldots \ldots$ & 233 & 266 & 66 & 15 & -- & -- & 3.4 & 26.0 & -6.8 \\
\hline Mean..... & $\overline{213}$ & $\overline{267}$ & 86 & $\overline{24}$ & $\overline{--}$ & 5.3 & $\overline{1.7}$ & $\overline{20.5}$ & -8.3 \\
\hline \multicolumn{10}{|c|}{ January 1986} \\
\hline $\begin{array}{l}1 \ldots \ldots \cdots \\
2 \ldots \ldots \cdots \\
3 \ldots \ldots \\
4 \ldots \ldots \\
5 \ldots \ldots \cdots\end{array}$ & $\begin{array}{l}213 \\
230 \\
214 \\
265 \\
269\end{array}$ & $\begin{array}{l}258 \\
261 \\
268 \\
289 \\
278\end{array}$ & $\begin{array}{l}89 \\
57 \\
99 \\
69 \\
33\end{array}$ & $\begin{array}{r}23 \\
18 \\
28 \\
18 \\
2\end{array}$ & $\begin{array}{l}-- \\
-- \\
-- \\
--\end{array}$ & $\begin{array}{l}-- \\
-- \\
-- \\
--\end{array}$ & $\begin{array}{l}1.6 \\
1.8 \\
3.7 \\
7.4 \\
8.7\end{array}$ & $\begin{array}{l}23.8 \\
20.4 \\
24.9 \\
16.4 \\
12.6\end{array}$ & $\begin{array}{r}-9.2 \\
-9.4 \\
-8.7 \\
.6 \\
6.8\end{array}$ \\
\hline $\begin{array}{r}6 \ldots \ldots \cdots \cdots \\
7 \ldots \ldots \cdots \cdots \\
8 \ldots \ldots \cdots \\
9 \ldots \ldots \cdots \\
10 \ldots \ldots\end{array}$ & $\begin{array}{l}212 \\
171 \\
176 \\
197 \\
204\end{array}$ & $\begin{array}{l}267 \\
256 \\
248 \\
266 \\
271\end{array}$ & $\begin{array}{r}95 \\
110 \\
102 \\
111 \\
90\end{array}$ & $\begin{array}{l}26 \\
33 \\
26 \\
32 \\
28\end{array}$ & $\begin{array}{l}-- \\
-- \\
-- \\
--\end{array}$ & $\begin{array}{l}-- \\
-- \\
-- \\
--\end{array}$ & $\begin{array}{r}8.0 \\
-.7 \\
-2.5 \\
-3.1 \\
1.9\end{array}$ & $\begin{array}{l}15.8 \\
16.7 \\
22.8 \\
25.4 \\
28.1\end{array}$ & $\begin{array}{r}-6.5 \\
-10.5 \\
-14.0 \\
-12.2 \\
-10.2\end{array}$ \\
\hline $\begin{array}{l}11 \ldots \cdots \cdots \\
12 \ldots \cdots \cdots\end{array}$ & $\begin{array}{l}207 \\
204 \\
-\end{array}$ & $\begin{array}{l}272 \\
273 \\
--\end{array}$ & $\begin{array}{l}104 \\
108 \\
--\end{array}$ & $\begin{array}{l}28 \\
30 \\
--\end{array}$ & $\begin{array}{l}-- \\
--\end{array}$ & $\begin{array}{l}-- \\
--\end{array}$ & $\begin{array}{l}2.2 \\
2.1 \\
--\end{array}$ & $\begin{array}{l}28.8 \\
29.3\end{array}$ & $\begin{array}{l}-9.8 \\
-9.9\end{array}$ \\
\hline $14 \ldots \ldots \cdots \cdots$ & $\begin{array}{l}241 \\
254\end{array}$ & $\begin{array}{l}266 \\
277\end{array}$ & $\begin{array}{l}21 \\
55\end{array}$ & $\begin{array}{l}10 \\
12\end{array}$ & -- & -- & $\begin{array}{l}2.5 \\
5.3\end{array}$ & $\begin{array}{l}19.2 \\
22.6\end{array}$ & $\begin{array}{l}-8.3 \\
-4.4\end{array}$ \\
\hline $\begin{array}{l}16 \ldots \ldots \cdots \\
17 \ldots \ldots \cdots \cdots \\
18 \ldots \ldots \cdots \\
19 \ldots \ldots \cdots \\
20 \ldots \ldots\end{array}$ & $\begin{array}{l}220 \\
216 \\
218 \\
217 \\
199\end{array}$ & $\begin{array}{l}264 \\
267 \\
283 \\
275 \\
274\end{array}$ & $\begin{array}{r}100 \\
111 \\
103 \\
96 \\
118\end{array}$ & $\begin{array}{l}20 \\
25 \\
30 \\
26 \\
34\end{array}$ & $\begin{array}{l}-- \\
-- \\
-- \\
--\end{array}$ & $\begin{array}{l}-- \\
-- \\
-- \\
--\end{array}$ & $\begin{array}{l}2.5 \\
2.3 \\
4.7 \\
3.3 \\
3.1\end{array}$ & $\begin{array}{l}25.0 \\
26.0 \\
30.8 \\
28.4 \\
25.2\end{array}$ & $\begin{array}{l}-7.0 \\
-8.7 \\
-8.1 \\
-9.6 \\
-8.1\end{array}$ \\
\hline $\begin{array}{l}21 \ldots \ldots \cdots \cdots \\
22 \ldots \ldots \cdots \cdots \\
23 \ldots \ldots \cdots \\
24 \ldots \ldots \cdots \\
25 \ldots \ldots \\
25 \ldots \cdots\end{array}$ & $\begin{array}{l}-- \\
-- \\
\overline{196} \\
199\end{array}$ & $\begin{array}{r}-- \\
-- \\
-- \\
276 \\
274\end{array}$ & $\begin{array}{l}-- \\
-- \\
-- \\
-- \\
--\end{array}$ & $\begin{array}{l}-- \\
-- \\
-- \\
41 \\
38\end{array}$ & $\begin{array}{l}-- \\
-- \\
-- \\
-- \\
--\end{array}$ & $\begin{array}{l}-- \\
-- \\
-- \\
--\end{array}$ & $\begin{array}{l}-- \\
-- \\
-- \\
3.5 \\
2.5\end{array}$ & $\begin{array}{c}-- \\
\overline{--} \\
27.0 \\
27.2\end{array}$ & $\begin{array}{c}-- \\
\overline{-} \\
-9.6 \\
-12.2\end{array}$ \\
\hline $\begin{array}{l}26 \ldots \ldots \ldots \\
27 \ldots \ldots \cdots \cdots \\
28 \ldots \ldots \cdots \cdots \\
29 \ldots \ldots \\
30 \ldots \ldots \\
30 \ldots \cdots\end{array}$ & $\begin{array}{l}199 \\
199 \\
207 \\
224 \\
279\end{array}$ & $\begin{array}{l}274 \\
274 \\
276 \\
278 \\
274\end{array}$ & $\begin{array}{l}-- \\
-- \\
-- \\
-- \\
--\end{array}$ & $\begin{array}{l}36 \\
36 \\
37 \\
32 \\
--\end{array}$ & $\begin{array}{l}-- \\
-- \\
-- \\
--\end{array}$ & $\begin{array}{l}-- \\
-- \\
-- \\
-- \\
--\end{array}$ & $\begin{array}{l}2.3 \\
2.6 \\
3.5 \\
4.8 \\
7.2\end{array}$ & $\begin{array}{l}31.2 \\
32.1 \\
30.5 \\
30.4 \\
11.1\end{array}$ & $\begin{array}{r}-12.7 \\
-12.6 \\
-11.1 \\
-10.1 \\
4.1\end{array}$ \\
\hline $31 \ldots \ldots \ldots$ & 264 & 282 & -- & 8 & -- & -- & 8.3 & 17.3 & 4.4 \\
\hline Mean..... & $\overline{218}$ & $\overline{271}$ & $\overline{88}$ & $\overrightarrow{26}$ & $\overrightarrow{--}$ & $\overline{--}$ & $\overline{3.3}$ & $\overline{24.0}$ & -7.7 \\
\hline
\end{tabular}


Table 2. Radiation and temperature field data for crater 1, May 1985 through June $1986-$ Continued

\begin{tabular}{|c|c|c|c|c|c|c|c|c|c|}
\hline \multirow{3}{*}{ Date } & \multicolumn{5}{|c|}{$\begin{array}{l}\text { Daily mean radiation } \\
\text { (watts per square meter) }\end{array}$} & \multicolumn{4}{|c|}{$\begin{array}{c}\text { Temperature } \\
\text { (degrees Celsius) }\end{array}$} \\
\hline & \multicolumn{2}{|c|}{ Long wave } & \multicolumn{2}{|c|}{ Short wave } & \multirow{2}{*}{ Net } & \multirow{2}{*}{$\begin{array}{c}\text { Daily mean } \\
\text { air } \\
\text { temperature }\end{array}$} & \multicolumn{2}{|c|}{ Soil surface } & temperature \\
\hline & In & out & In & Out & & & Mean & Maximum & Minimum \\
\hline \multicolumn{10}{|c|}{ Fabruary 1986} \\
\hline $\begin{array}{l}1 \ldots \ldots \\
2 \ldots \ldots \\
3 \ldots \ldots \\
4 \ldots \ldots \\
5 \ldots \ldots\end{array}$ & $\begin{array}{r}227 \\
210 \\
203 \\
-- \\
-\end{array}$ & $\begin{array}{r}282 \\
263 \\
257 \\
-- \\
--\end{array}$ & $\begin{array}{l}-- \\
-- \\
-- \\
-- \\
--\end{array}$ & $\begin{array}{l}15 \\
22 \\
24 \\
-- \\
--\end{array}$ & $\begin{array}{l}-- \\
-- \\
-- \\
-- \\
--\end{array}$ & $\begin{array}{l}-- \\
-- \\
-- \\
-- \\
--\end{array}$ & $\begin{array}{l}7.5 \\
4.1 \\
4.0 \\
-- \\
--\end{array}$ & $\begin{array}{l}20.5 \\
17.4 \\
18.4 \\
-- \\
--\end{array}$ & $\begin{array}{l}-0.1 \\
-1.7 \\
-4.3 \\
-- \\
--\end{array}$ \\
\hline $\begin{array}{r}6 \ldots \ldots \ldots \\
7 \ldots \ldots \\
8 \ldots \ldots \ldots \\
9 \ldots \ldots \\
10 \ldots \ldots \ldots\end{array}$ & $\begin{array}{r}-- \\
-- \\
-- \\
-- \\
155\end{array}$ & $\begin{array}{r}-- \\
-- \\
-- \\
-- \\
207\end{array}$ & $\begin{array}{l}-- \\
-- \\
-- \\
-- \\
--\end{array}$ & $\begin{array}{l}-- \\
-- \\
-- \\
--\end{array}$ & $\begin{array}{l}-- \\
-- \\
-- \\
-- \\
--\end{array}$ & $\begin{array}{l}-- \\
-- \\
-- \\
-- \\
-0.5\end{array}$ & $\begin{array}{l}-- \\
-- \\
-- \\
-- \\
-2.4\end{array}$ & $\begin{array}{c}-- \\
-- \\
-- \\
-- \\
20.9\end{array}$ & $\begin{array}{c}-- \\
-- \\
-- \\
-- \\
-14.8\end{array}$ \\
\hline $\begin{array}{l}11 \ldots \ldots \\
12 \ldots \ldots \\
13 \ldots \ldots \\
14 \ldots \ldots \\
15 \ldots \ldots\end{array}$ & $\begin{array}{l}189 \\
237 \\
259 \\
277 \\
264\end{array}$ & $\begin{array}{l}245 \\
254 \\
277 \\
278 \\
277\end{array}$ & $\begin{array}{l}-- \\
-- \\
-- \\
-- \\
--\end{array}$ & $\begin{array}{r}18 \\
4 \\
13 \\
12\end{array}$ & $\begin{array}{r}-17 \\
-19 \\
11 \\
-25 \\
30\end{array}$ & $\begin{array}{r}-2.2 \\
1.1 \\
8.0 \\
8.3 \\
8.5\end{array}$ & $\begin{array}{r}-2.9 \\
.2 \\
6.6 \\
7.3 \\
7.5\end{array}$ & $\begin{array}{r}20.4 \\
9.5 \\
18.1 \\
16.9 \\
18.1\end{array}$ & $\begin{array}{r}-13.4 \\
-10.0 \\
2.6 \\
3.1 \\
2.4\end{array}$ \\
\hline $\begin{array}{l}16 \ldots \ldots \ldots \\
17 \ldots \ldots \\
18 \ldots \ldots \ldots \\
19 \ldots \ldots \ldots \\
20 \ldots \ldots \ldots\end{array}$ & $\begin{array}{l}228 \\
236 \\
260 \\
248 \\
194\end{array}$ & $\begin{array}{l}276 \\
271 \\
286 \\
283 \\
270\end{array}$ & $\begin{array}{l}-- \\
-- \\
-- \\
-- \\
--\end{array}$ & $\begin{array}{r}10 \\
9 \\
12 \\
11 \\
37\end{array}$ & $\begin{array}{r}23 \\
0 \\
3 \\
-5 \\
14\end{array}$ & $\begin{array}{l}7.9 \\
8.6 \\
9.2 \\
-- \\
--\end{array}$ & $\begin{array}{l}5.5 \\
5.7 \\
9.4 \\
9.1 \\
7.3\end{array}$ & $\begin{array}{l}18.4 \\
18.6 \\
26.9 \\
18.6 \\
18.4\end{array}$ & $\begin{array}{r}-2.3 \\
-2.5 \\
1.6 \\
1.2 \\
-.4\end{array}$ \\
\hline $\begin{array}{l}21 \ldots \ldots \ldots \\
22 \ldots \ldots \ldots \\
23 \ldots \ldots \ldots \\
24 \ldots \ldots \ldots \\
25 \ldots \ldots \ldots\end{array}$ & $\begin{array}{r}189 \\
206 \\
223 \\
-- \\
--\end{array}$ & $\begin{array}{r}275 \\
276 \\
294 \\
-- \\
--\end{array}$ & $\begin{array}{l}-- \\
-- \\
-- \\
-- \\
--\end{array}$ & $\begin{array}{l}46 \\
42 \\
43 \\
-- \\
--\end{array}$ & $\begin{array}{l}22 \\
22 \\
27 \\
-- \\
--\end{array}$ & $\begin{array}{l}-- \\
-- \\
-- \\
-- \\
--\end{array}$ & $\begin{array}{c}15.2 \\
1.6 \\
7.3 \\
-- \\
--\end{array}$ & $\begin{array}{l}26.9 \\
15.1 \\
13.2 \\
-- \\
--\end{array}$ & $\begin{array}{r}3.2 \\
-10.7 \\
-3.2 \\
-- \\
--\end{array}$ \\
\hline $\begin{array}{l}26 \ldots \ldots \ldots \ldots \\
27 \ldots \ldots \ldots \ldots \\
28 \ldots \ldots \ldots \ldots\end{array}$ & $\begin{array}{l}234 \\
234 \\
225\end{array}$ & $\begin{array}{l}318 \\
321 \\
314\end{array}$ & $\begin{array}{l}-- \\
-- \\
--\end{array}$ & $\begin{array}{l}48 \\
51 \\
50\end{array}$ & $\begin{array}{l}25 \\
26 \\
25\end{array}$ & $\begin{array}{l}-- \\
-- \\
--\end{array}$ & $\begin{array}{l}12 \cdot 9 \\
12 \cdot 5 \\
12 \cdot 1\end{array}$ & $\begin{array}{l}41.0 \\
38.6 \\
40.4\end{array}$ & $\begin{array}{l}-3.2 \\
-1.1 \\
-4.3\end{array}$ \\
\hline Mean..... & $\overline{225}$ & $\overline{276}$ & -- & $\overline{27}$ & 10 & 5.4 & $\overline{6.5}$ & $\overline{21.8}$ & -2.9 \\
\hline \multicolumn{10}{|c|}{ March 1986} \\
\hline $\begin{array}{l}1 \ldots \ldots \ldots \\
2 \ldots \ldots \\
3 \ldots \ldots \\
4 \ldots \ldots \\
5 \ldots \ldots \ldots\end{array}$ & $\begin{array}{l}236 \\
253 \\
245 \\
217 \\
194\end{array}$ & $\begin{array}{l}315 \\
298 \\
320 \\
320 \\
322\end{array}$ & $\begin{array}{l}-- \\
-- \\
-- \\
-- \\
--\end{array}$ & $\begin{array}{l}48 \\
16 \\
49 \\
50 \\
53\end{array}$ & $\begin{array}{r}26 \\
-10 \\
37 \\
26 \\
33\end{array}$ & $\begin{array}{l}-- \\
-- \\
-- \\
--\end{array}$ & $\begin{array}{r}12.5 \\
9.4 \\
12.9 \\
13.1 \\
12.6\end{array}$ & $\begin{array}{l}39.2 \\
29.7 \\
37.0 \\
41.0 \\
41.3\end{array}$ & $\begin{array}{r}-4.3 \\
1.1 \\
-.2 \\
-2.8 \\
-4.4\end{array}$ \\
\hline $\begin{array}{r}6 \ldots \ldots \ldots \\
7 \ldots \ldots \ldots \\
8 \ldots \ldots \ldots \\
9 \ldots \ldots \ldots \\
10 \ldots \ldots \ldots\end{array}$ & $\begin{array}{l}207 \\
216 \\
237 \\
203 \\
247\end{array}$ & $\begin{array}{l}316 \\
305 \\
297 \\
291 \\
273\end{array}$ & $\begin{array}{l}-- \\
-- \\
-- \\
-- \\
--\end{array}$ & $\begin{array}{l}50 \\
35 \\
21 \\
43 \\
--\end{array}$ & $\begin{array}{r}30 \\
16 \\
7 \\
59 \\
-14\end{array}$ & $\begin{array}{l}-- \\
-- \\
-- \\
-- \\
--\end{array}$ & $\begin{array}{r}11.9 \\
10.5 \\
8.9 \\
8.8 \\
5.8\end{array}$ & $\begin{array}{l}40.0 \\
37.5 \\
29.8 \\
30.2 \\
12.5\end{array}$ & $\begin{array}{r}-4.2 \\
-2.7 \\
.3 \\
-3.9 \\
2.2\end{array}$ \\
\hline $\begin{array}{l}11 \ldots \ldots \ldots \ldots \\
12 \ldots \ldots \ldots \\
13 \ldots \ldots \ldots \\
14 \ldots \ldots \ldots \\
15 \ldots \ldots \ldots \ldots\end{array}$ & $\begin{array}{l}-- \\
-- \\
-- \\
-- \\
--\end{array}$ & $\begin{array}{l}279 \\
267 \\
267 \\
273 \\
270\end{array}$ & $\begin{array}{l}-- \\
-- \\
-- \\
-- \\
--\end{array}$ & $\begin{array}{l}29 \\
16 \\
31 \\
31 \\
43\end{array}$ & $\begin{array}{r}68 \\
4 \\
25 \\
68 \\
63\end{array}$ & $\begin{array}{l}-- \\
-- \\
-- \\
-- \\
--\end{array}$ & $\begin{array}{l}5.7 \\
2.8 \\
4.9 \\
6.7 \\
6.2\end{array}$ & $\begin{array}{l}23.0 \\
25.8 \\
25.3 \\
24.7 \\
29.1\end{array}$ & $\begin{array}{l}-2.9 \\
-5.6 \\
-6.8 \\
-3.9 \\
-6.6\end{array}$ \\
\hline $\begin{array}{l}16 \ldots \ldots \ldots \\
17 \ldots \ldots \ldots \\
18 \ldots \ldots \ldots \\
19 \ldots \ldots \ldots \\
20 \ldots \ldots \ldots\end{array}$ & $\begin{array}{l}-- \\
-- \\
-- \\
-- \\
--\end{array}$ & $\begin{array}{r}258 \\
-- \\
-- \\
-- \\
302\end{array}$ & $\begin{array}{r}-- \\
-- \\
-- \\
-- \\
215\end{array}$ & $\begin{array}{l}13 \\
-- \\
-- \\
-- \\
57\end{array}$ & $\begin{array}{l}34 \\
-- \\
-- \\
-- \\
50\end{array}$ & $\begin{array}{l}-- \\
-- \\
-- \\
-- \\
--\end{array}$ & $\begin{array}{c}5.3 \\
-- \\
-- \\
-- \\
10.9\end{array}$ & $\begin{array}{c}20.2 \\
-- \\
-- \\
-- \\
39.6\end{array}$ & $\begin{array}{l}-.3 \\
-- \\
-- \\
-- \\
-5.3\end{array}$ \\
\hline
\end{tabular}


Table 2. Radiation and temperature field data for crater 1, May 1985 through June $1986-$ Continued

\begin{tabular}{|c|c|c|c|c|c|c|c|c|c|}
\hline \multirow{3}{*}{ Date } & \multicolumn{5}{|c|}{$\begin{array}{l}\text { Daily mean radiation } \\
\text { (watts per square meter) }\end{array}$} & \multicolumn{4}{|c|}{$\begin{array}{c}\text { Temperature } \\
\text { (degrees Celsius) }\end{array}$} \\
\hline & \multicolumn{2}{|c|}{ Long wave } & \multicolumn{2}{|c|}{ Short wave } & \multirow{2}{*}{ Net } & \multirow{2}{*}{$\begin{array}{c}\text { Daily mean } \\
\text { air } \\
\text { temperature }\end{array}$} & \multirow{2}{*}{ Soil } & \multirow{2}{*}{$\frac{\text { surface } t}{\text { Maximum }}$} & temperature \\
\hline & In & out & In & out & & & & & Minimum \\
\hline \multicolumn{10}{|c|}{ March 1986-Continued } \\
\hline $\begin{array}{l}21 \ldots \ldots \cdots \cdots \\
22 \ldots \ldots \cdots \\
23 \ldots \ldots \cdots \\
24 \ldots \ldots \\
25 \ldots \ldots \cdots\end{array}$ & $\begin{array}{l}-- \\
-- \\
-- \\
--\end{array}$ & $\begin{array}{l}302 \\
306 \\
304 \\
311 \\
320\end{array}$ & $\begin{array}{l}212 \\
200 \\
166 \\
168 \\
165\end{array}$ & $\begin{array}{l}60 \\
62 \\
45 \\
50 \\
61\end{array}$ & $\begin{array}{l}59 \\
57 \\
35 \\
43 \\
56\end{array}$ & $\begin{array}{l}-- \\
-- \\
-- \\
--\end{array}$ & $\begin{array}{l}11.1 \\
11.7 \\
11.4 \\
13.3 \\
13.8\end{array}$ & $\begin{array}{l}40.2 \\
40.9 \\
39.2 \\
39.1 \\
44.6\end{array}$ & $\begin{array}{r}-5.9 \\
-6.6 \\
-5.5 \\
.7 \\
-4.7\end{array}$ \\
\hline $\begin{array}{l}26 \ldots \ldots \ldots \\
27 \ldots \ldots \ldots \\
28 \ldots \ldots \ldots \\
29 \ldots \ldots \\
30 \ldots \ldots\end{array}$ & $\begin{array}{l}-- \\
-- \\
=- \\
--\end{array}$ & $\begin{array}{l}325 \\
326 \\
330 \\
322 \\
330\end{array}$ & $\begin{array}{r}211 \\
196 \\
178 \\
84 \\
214\end{array}$ & $\begin{array}{l}61 \\
62 \\
49 \\
16 \\
57\end{array}$ & $\begin{array}{r}44 \\
20 \\
42 \\
0 \\
61\end{array}$ & $\begin{array}{l}-- \\
-- \\
-- \\
-- \\
--\end{array}$ & $\begin{array}{l}15.1 \\
15.6 \\
16.9 \\
14.8 \\
17.0\end{array}$ & $\begin{array}{l}46.0 \\
47.1 \\
49.5 \\
33.9 \\
45.0\end{array}$ & $\begin{array}{r}-3.5 \\
-3.8 \\
-2.2 \\
5.2 \\
-.6\end{array}$ \\
\hline $31 \ldots \ldots \ldots$ & -- & 311 & 124 & 25 & 27 & -- & 13.1 & 40.9 & -.6 \\
\hline Mean..... & $\overline{226}$ & $\overline{302}$ & $\overline{178}$ & $\overline{42}$ & 34 & -- & $\overline{10.8}$ & $\overline{35.4}$ & -2.8 \\
\hline \multicolumn{10}{|c|}{ April 1986} \\
\hline $\begin{array}{l}1 \ldots \ldots \ldots \\
2 \ldots \ldots \ldots \\
3 \ldots \ldots \\
4 \ldots \ldots \\
5 \ldots \ldots\end{array}$ & $\begin{array}{l}269 \\
204 \\
212 \\
225 \\
233\end{array}$ & $\begin{array}{l}322 \\
296 \\
309 \\
307 \\
306\end{array}$ & $\begin{array}{l}192 \\
230 \\
215 \\
189 \\
169\end{array}$ & $\begin{array}{l}49 \\
69 \\
69 \\
61 \\
50\end{array}$ & $\begin{array}{l}69 \\
64 \\
63 \\
57 \\
40\end{array}$ & $\begin{array}{r}14.7 \\
7.9 \\
10.5 \\
9.7 \\
10.6\end{array}$ & $\begin{array}{l}15.4 \\
10.5 \\
12.7 \\
13.5 \\
12.2\end{array}$ & $\begin{array}{l}37.7 \\
31.9 \\
34.9 \\
39.2 \\
38.6\end{array}$ & $\begin{array}{r}3.3 \\
-2.7 \\
-3.4 \\
-4.0 \\
-2.5\end{array}$ \\
\hline $\begin{array}{r}6 \ldots \ldots \cdots \\
7 \ldots \ldots \cdots \\
8 \ldots \ldots \cdots \\
9 \ldots \ldots \\
10 \ldots \ldots\end{array}$ & $\begin{array}{l}256 \\
242 \\
237 \\
236 \\
250\end{array}$ & $\begin{array}{l}281 \\
290 \\
304 \\
319 \\
322\end{array}$ & $\begin{array}{r}74 \\
193 \\
215 \\
196 \\
183\end{array}$ & $\begin{array}{l}15 \\
43 \\
53 \\
56 \\
50\end{array}$ & $\begin{array}{l}-5 \\
76 \\
77 \\
64 \\
54\end{array}$ & $\begin{array}{r}7.1 \\
8.5 \\
10.0 \\
11.8 \\
12.3\end{array}$ & $\begin{array}{r}8.4 \\
10.7 \\
13.0 \\
14.9 \\
15.2\end{array}$ & $\begin{array}{l}26.9 \\
30.9 \\
43.7 \\
44.9 \\
45.4\end{array}$ & $\begin{array}{r}-1.2 \\
.0 \\
-.4 \\
-2.6 \\
.7\end{array}$ \\
\hline $\begin{array}{l}11 \ldots \ldots \\
12 \ldots \ldots \cdots \\
13 \ldots \ldots \cdots \\
14 \ldots \ldots \\
15 \ldots \ldots\end{array}$ & $\begin{array}{l}232 \\
227 \\
202 \\
244 \\
237\end{array}$ & $\begin{array}{l}-- \\
-- \\
-- \\
-- \\
331\end{array}$ & $\begin{array}{l}235 \\
209 \\
245 \\
169 \\
221\end{array}$ & $\begin{array}{l}68 \\
73 \\
80 \\
47 \\
72\end{array}$ & $\begin{array}{l}75 \\
81 \\
74 \\
37 \\
74\end{array}$ & $\begin{array}{r}12.7 \\
12.0 \\
9.0 \\
10.2 \\
12.5\end{array}$ & $\begin{array}{l}16.2 \\
12.7 \\
11.9 \\
12.4 \\
14.4\end{array}$ & $\begin{array}{l}46.6 \\
39.4 \\
39.5 \\
41.5 \\
38.6\end{array}$ & $\begin{array}{l}-2.2 \\
-2.3 \\
-6.0 \\
-3.6 \\
-3.0\end{array}$ \\
\hline $\begin{array}{l}16 \ldots \ldots \ldots \\
17 \ldots \ldots \cdots \\
18 \ldots \ldots \\
19 \ldots \ldots \\
20 \ldots \ldots\end{array}$ & $\begin{array}{l}230 \\
215 \\
203 \\
219 \\
253\end{array}$ & $\begin{array}{l}301 \\
308 \\
312 \\
326 \\
353\end{array}$ & $\begin{array}{l}128 \\
216 \\
257 \\
236 \\
249\end{array}$ & $\begin{array}{l}37 \\
60 \\
77 \\
74 \\
74\end{array}$ & $\begin{array}{l}16 \\
58 \\
75 \\
72 \\
77\end{array}$ & $\begin{array}{r}7.7 \\
8.5 \\
8.9 \\
11.1 \\
17.2\end{array}$ & $\begin{array}{r}9.2 \\
11.4 \\
11.7 \\
14.2 \\
18.8\end{array}$ & $\begin{array}{l}32.9 \\
44.9 \\
40.6 \\
44.9 \\
47.9\end{array}$ & $\begin{array}{l}-4.7 \\
-8.1 \\
-5.1 \\
-6.8 \\
-1.0\end{array}$ \\
\hline $\begin{array}{l}21 \ldots \ldots \cdots \\
22 \ldots \ldots \cdots \\
23 \ldots \ldots \cdots \\
24 \ldots \ldots \\
25 \ldots \ldots \cdots\end{array}$ & $\begin{array}{l}251 \\
255 \\
247 \\
254 \\
237\end{array}$ & $\begin{array}{l}331 \\
352 \\
342 \\
330 \\
334\end{array}$ & $\begin{array}{l}187 \\
233 \\
243 \\
156 \\
259\end{array}$ & $\begin{array}{l}67 \\
72 \\
72 \\
46 \\
76\end{array}$ & $\begin{array}{l}63 \\
75 \\
78 \\
34 \\
87\end{array}$ & $\begin{array}{l}18.1 \\
16.9 \\
16.0 \\
14.4 \\
14.2\end{array}$ & $\begin{array}{l}14.3 \\
19.5 \\
15.0 \\
16.6 \\
---\end{array}$ & $\begin{array}{l}48.5 \\
48.7 \\
33.6 \\
28.7 \\
--\end{array}$ & $\begin{array}{r}.6 \\
1.0 \\
-1.2 \\
-- \\
--\end{array}$ \\
\hline $\begin{array}{l}26 \ldots \ldots \ldots \\
27 \ldots \ldots \cdots \\
28 \ldots \ldots \cdots \\
29 \ldots \ldots \cdots \\
30 \ldots \ldots \cdots\end{array}$ & $\begin{array}{l}210 \\
229 \\
248 \\
252 \\
240\end{array}$ & $\begin{array}{l}319 \\
338 \\
357 \\
357 \\
345\end{array}$ & $\begin{array}{l}266 \\
274 \\
295 \\
255 \\
236\end{array}$ & $\begin{array}{l}80 \\
79 \\
87 \\
77 \\
72\end{array}$ & $\begin{array}{r}82 \\
86 \\
107 \\
82 \\
73\end{array}$ & $\begin{array}{l}10.1 \\
12.3 \\
16.2 \\
17.4 \\
15.6\end{array}$ & $\begin{array}{l}-- \\
-- \\
-- \\
--\end{array}$ & $\begin{array}{l}-- \\
-- \\
-- \\
--\end{array}$ & $\begin{array}{l}-- \\
-- \\
-- \\
--\end{array}$ \\
\hline Mean...... & 235 & $\overline{323}$ & $\overline{214}$ & 64 & 66 & 12.1 & 13.5 & $\overline{39.6}$ & -2.4 \\
\hline
\end{tabular}


Table 2. Radiation and temperature field data for crater 1, May 1985 through June $1986-$ Continued

\begin{tabular}{|c|c|c|c|c|c|c|c|c|c|}
\hline \multirow{3}{*}{ Date } & \multicolumn{5}{|c|}{$\begin{array}{l}\text { Daily mean radiation } \\
\text { (watts per square meter) }\end{array}$} & \multicolumn{4}{|c|}{$\begin{array}{c}\text { Temperature } \\
\text { (degrees Celsius) }\end{array}$} \\
\hline & \multicolumn{2}{|c|}{ Long wave } & \multicolumn{2}{|c|}{ Short wave } & \multirow{2}{*}{ Net } & \multirow{2}{*}{$\begin{array}{c}\text { Daily mean } \\
\text { air } \\
\text { temperature }\end{array}$} & \multicolumn{2}{|c|}{ Soil surface $t$} & temperature \\
\hline & In & out & In & Out & & & Mean & Maximum & Minimum \\
\hline \multicolumn{10}{|c|}{ May 1986} \\
\hline $\begin{array}{l}1 \ldots \ldots \\
2 \ldots \ldots \\
3 \ldots \ldots \\
4 \ldots \ldots \\
5 \ldots \ldots\end{array}$ & $\begin{array}{l}263 \\
251 \\
252 \\
233 \\
242\end{array}$ & $\begin{array}{l}349 \\
358 \\
351 \\
336 \\
327\end{array}$ & $\begin{array}{l}190 \\
242 \\
249 \\
237 \\
190\end{array}$ & $\begin{array}{l}65 \\
84 \\
86 \\
71 \\
64\end{array}$ & $\begin{array}{r}65 \\
100 \\
107 \\
86 \\
54\end{array}$ & $\begin{array}{l}-- \\
-- \\
-- \\
-- \\
--\end{array}$ & $\begin{array}{c}-- \\
-- \\
19.8 \\
18.1 \\
15.8\end{array}$ & $\begin{array}{l}-- \\
-- \\
45.0 \\
48.6 \\
47.4\end{array}$ & $\begin{array}{r}-- \\
-- \\
1.3 \\
.5 \\
-5.7\end{array}$ \\
\hline $\begin{array}{c}6 \ldots \ldots \ldots \\
7 \ldots \ldots \ldots \\
8 \ldots \ldots \ldots \\
9 \ldots \ldots \ldots \\
10 \ldots \ldots \ldots\end{array}$ & $\begin{array}{l}254 \\
228 \\
224 \\
219 \\
238\end{array}$ & $\begin{array}{l}283 \\
311 \\
315 \\
333 \\
335\end{array}$ & $\begin{array}{r}58 \\
289 \\
240 \\
281 \\
256\end{array}$ & $\begin{array}{l}6 \\
72 \\
64 \\
76 \\
74\end{array}$ & $\begin{array}{r}-6 \\
121 \\
79 \\
97 \\
104\end{array}$ & $\begin{array}{l}-- \\
-- \\
-- \\
14.1 \\
16.1\end{array}$ & $\begin{array}{r}7.9 \\
13.7 \\
14.9 \\
18.3 \\
18.2\end{array}$ & $\begin{array}{l}23.2 \\
40.6 \\
45.6 \\
49.6 \\
45.2\end{array}$ & $\begin{array}{r}2.0 \\
-1.9 \\
-3.5 \\
-3.1 \\
-.2\end{array}$ \\
\hline $\begin{array}{l}11 \ldots \ldots \ldots \\
12 \ldots \ldots \\
13 \ldots \ldots \\
14 \ldots \ldots \ldots \\
15 \ldots \ldots\end{array}$ & $\begin{array}{l}233 \\
241 \\
253 \\
272 \\
275\end{array}$ & $\begin{array}{l}342 \\
353 \\
366 \\
368 \\
379\end{array}$ & $\begin{array}{l}248 \\
275 \\
282 \\
247 \\
266\end{array}$ & $\begin{array}{l}78 \\
78 \\
77 \\
68 \\
70\end{array}$ & $\begin{array}{l}97 \\
93 \\
92 \\
82 \\
87\end{array}$ & $\begin{array}{l}15.1 \\
16.0 \\
18.5 \\
19.6 \\
21.2\end{array}$ & $\begin{array}{l}20.0 \\
21.7 \\
23.4 \\
23.7 \\
25.9\end{array}$ & $\begin{array}{l}49.8 \\
52.8 \\
54.6 \\
55.9 \\
56.3\end{array}$ & $\begin{array}{r}-.5 \\
-1.6 \\
.4 \\
2.7 \\
9.1\end{array}$ \\
\hline $\begin{array}{l}16 \ldots \ldots \ldots \\
17 \ldots \ldots \ldots \\
18 \ldots \ldots \\
19 \ldots \ldots \ldots \\
20 \ldots \ldots\end{array}$ & $\begin{array}{r}239 \\
235 \\
247 \\
-- \\
281\end{array}$ & $\begin{array}{r}362 \\
362 \\
371 \\
-- \\
385\end{array}$ & $\begin{array}{r}295 \\
290 \\
298 \\
-- \\
280\end{array}$ & $\begin{array}{l}81 \\
82 \\
82 \\
-2 \\
82\end{array}$ & $\begin{array}{r}91 \\
92 \\
42 \\
-- \\
100\end{array}$ & $\begin{array}{l}19.3 \\
17.1 \\
18.3 \\
-- \\
22.7\end{array}$ & $\begin{array}{c}23.3 \\
22.5 \\
23.5 \\
-- \\
25.1\end{array}$ & $\begin{array}{l}52.2 \\
52.9 \\
55.7 \\
-- \\
51.2\end{array}$ & $\begin{array}{r}3.7 \\
.1 \\
-.8 \\
-- \\
5.1\end{array}$ \\
\hline $\begin{array}{l}21 \ldots \ldots \ldots \\
22 \ldots \ldots \ldots \\
23 \ldots \ldots \ldots \\
24 \ldots \ldots \ldots \\
25 \ldots \ldots \ldots\end{array}$ & $\begin{array}{l}268 \\
243 \\
258 \\
262 \\
270\end{array}$ & $\begin{array}{l}379 \\
363 \\
366 \\
383 \\
388\end{array}$ & $\begin{array}{l}291 \\
279 \\
269 \\
286 \\
291\end{array}$ & $\begin{array}{l}86 \\
82 \\
78 \\
83 \\
84\end{array}$ & $\begin{array}{r}108 \\
83 \\
86 \\
90 \\
92\end{array}$ & $\begin{array}{l}20.6 \\
17.1 \\
16.5 \\
20.0 \\
22.1\end{array}$ & $\begin{array}{l}24.0 \\
21.6 \\
22.0 \\
25.2 \\
26.8\end{array}$ & $\begin{array}{l}47.7 \\
52.1 \\
51.5 \\
56.4 \\
58.4\end{array}$ & $\begin{array}{r}8.8 \\
2.2 \\
-1.2 \\
1.2 \\
2.7\end{array}$ \\
\hline $\begin{array}{l}26 \ldots \ldots \ldots \\
27 \ldots \ldots \ldots \\
28 \ldots \ldots \ldots \\
29 \ldots \ldots \ldots \\
30 \ldots \ldots\end{array}$ & $\begin{array}{l}272 \\
267 \\
252 \\
264 \\
265\end{array}$ & $\begin{array}{l}392 \\
394 \\
395 \\
394 \\
389\end{array}$ & $\begin{array}{l}290 \\
297 \\
264 \\
247 \\
294\end{array}$ & $\begin{array}{l}84 \\
84 \\
77 \\
66 \\
82\end{array}$ & $\begin{array}{r}92 \\
91 \\
82 \\
65 \\
100\end{array}$ & $\begin{array}{l}23.2 \\
24.0 \\
25.5 \\
25.8 \\
26.6\end{array}$ & $\begin{array}{l}27.6 \\
27.1 \\
28.5 \\
29.7 \\
27.8\end{array}$ & $\begin{array}{l}57.7 \\
58.5 \\
57.9 \\
62.3 \\
59.2\end{array}$ & $\begin{array}{l}4.4 \\
6.2 \\
7.2 \\
8.0 \\
8.8\end{array}$ \\
\hline $31 \ldots \ldots \ldots$ & -- & -- & -- & -- & -- & -- & - & -- & -- \\
\hline \multirow[t]{2}{*}{ Mean..... } & $\overline{252}$ & $\overline{360}$ & $\overline{259}$ & $\overline{75}$ & $\overline{85}$ & $\overline{20.0}$ & $\overline{22.1}$ & $\overline{51.4}$ & $\overline{2.1}$ \\
\hline & ' & & & Ju & 1986 & & & & \\
\hline $\begin{array}{l}1 \ldots \ldots \\
2 \ldots \ldots \\
3 \ldots \ldots \\
4 \ldots \ldots \\
5 \ldots \ldots\end{array}$ & $\begin{array}{l}-- \\
-- \\
-- \\
-- \\
--\end{array}$ & $\begin{array}{l}420 \\
408 \\
405 \\
394 \\
382\end{array}$ & $\begin{array}{l}285 \\
251 \\
289 \\
256 \\
308\end{array}$ & $\begin{array}{l}77 \\
69 \\
78 \\
74 \\
87\end{array}$ & $\begin{array}{r}87 \\
80 \\
98 \\
77 \\
102\end{array}$ & $\begin{array}{l}29.1 \\
27.2 \\
26.1 \\
25.5 \\
23.0\end{array}$ & $\begin{array}{l}34.1 \\
31.7 \\
31.2 \\
28.5 \\
26.8\end{array}$ & $\begin{array}{l}61.1 \\
59.2 \\
59.1 \\
55.7 \\
52.9\end{array}$ & $\begin{array}{r}13.2 \\
14.6 \\
10.2 \\
8.8 \\
6.0\end{array}$ \\
\hline $\begin{array}{c}6 \ldots \ldots \ldots \\
7 \ldots \ldots \\
8 \ldots \ldots \\
9 \ldots \ldots \\
10 \ldots \ldots\end{array}$ & $\begin{array}{l}-- \\
-- \\
-- \\
--\end{array}$ & $\begin{array}{l}375 \\
372 \\
383 \\
385 \\
385\end{array}$ & $\begin{array}{l}304 \\
302 \\
289 \\
307 \\
303\end{array}$ & $\begin{array}{l}82 \\
83 \\
81 \\
86 \\
84\end{array}$ & $\begin{array}{r}96 \\
102 \\
102 \\
92 \\
91\end{array}$ & $\begin{array}{l}20.7 \\
21.4 \\
22.8 \\
22.8 \\
22.4\end{array}$ & $\begin{array}{l}26.0 \\
25.3 \\
26.8 \\
27.0 \\
27.7\end{array}$ & $\begin{array}{l}55.9 \\
50.8 \\
52.3 \\
56.4 \\
57.0\end{array}$ & $\begin{array}{r}2.9 \\
4.4 \\
12.2 \\
4.4 \\
4.7\end{array}$ \\
\hline $\begin{array}{l}11 \ldots \ldots \ldots \\
12 \ldots \ldots \\
13 \ldots \ldots \\
14 \ldots \ldots \\
15 \ldots \ldots\end{array}$ & $\begin{array}{l}-- \\
-- \\
-- \\
-- \\
--\end{array}$ & $\begin{array}{l}389 \\
385 \\
391 \\
384 \\
379\end{array}$ & $\begin{array}{l}302 \\
301 \\
304 \\
287 \\
266\end{array}$ & $\begin{array}{l}84 \\
85 \\
86 \\
82 \\
77\end{array}$ & $\begin{array}{l}94 \\
95 \\
91 \\
87 \\
74\end{array}$ & $\begin{array}{l}23.0 \\
22.9 \\
22.9 \\
23.4 \\
23.4\end{array}$ & $\begin{array}{l}28.2 \\
27.2 \\
27.8 \\
26.6 \\
25.0\end{array}$ & $\begin{array}{l}57.2 \\
56.3 \\
58.6 \\
54.5 \\
54.6\end{array}$ & $\begin{array}{l}4.5 \\
5.7 \\
3.7 \\
4.5 \\
3.8\end{array}$ \\
\hline
\end{tabular}


Table 2. Radiation and temperature field data for crater 1, May 1985 through June 1986 Continued

\begin{tabular}{|c|c|c|c|c|c|c|c|c|c|}
\hline \multirow{3}{*}{ Date } & \multicolumn{5}{|c|}{$\begin{array}{l}\text { Daily mean radiation } \\
\text { (watts per square meter) }\end{array}$} & \multicolumn{4}{|c|}{$\begin{array}{c}\text { Temperature } \\
\text { (degrees Celsius) }\end{array}$} \\
\hline & \multicolumn{2}{|c|}{ Long wave } & \multicolumn{2}{|c|}{ Short wave } & \multirow{2}{*}{ Net } & \multirow{2}{*}{$\begin{array}{c}\text { Daily mean } \\
\text { air } \\
\text { temperature }\end{array}$} & \multicolumn{2}{|c|}{ Soil surface } & temperature \\
\hline & In & Out & In & out & & & Mean & Maximum & Minimum \\
\hline \multicolumn{10}{|c|}{ June 1986-Continued } \\
\hline $\begin{array}{l}16 \ldots \ldots \ldots \\
17 \ldots \ldots \\
18 \ldots \ldots \ldots \\
19 \ldots \ldots \ldots \\
20 \ldots \ldots \ldots\end{array}$ & $\begin{array}{l}-- \\
-- \\
-- \\
-- \\
--\end{array}$ & $\begin{array}{l}-- \\
389 \\
388 \\
390 \\
388\end{array}$ & $\begin{array}{l}-\overline{3} \\
309 \\
309 \\
312\end{array}$ & $\begin{array}{l}-- \\
92 \\
91 \\
90 \\
92\end{array}$ & $\begin{array}{r}-- \\
104 \\
107 \\
98 \\
101\end{array}$ & $\begin{array}{l}-- \\
23.4 \\
23.2 \\
22.4 \\
19.8\end{array}$ & $\begin{array}{l}-- \\
26.8 \\
27.2 \\
27.2 \\
26.2\end{array}$ & $\begin{array}{l}-- \\
53.5 \\
54.5 \\
56.8 \\
55.5\end{array}$ & $\begin{array}{l}-- \\
3.4 \\
4.3 \\
4.1 \\
3.2\end{array}$ \\
\hline $\begin{array}{l}21 \ldots \ldots \ldots \\
22 \ldots \ldots \ldots \\
23 \ldots \ldots \ldots \\
24 \ldots \ldots \ldots \\
25 \ldots \ldots \ldots\end{array}$ & $\begin{array}{l}-- \\
-- \\
-- \\
--\end{array}$ & $\begin{array}{l}390 \\
396 \\
396 \\
418 \\
423\end{array}$ & $\begin{array}{l}306 \\
306 \\
274 \\
304 \\
302\end{array}$ & $\begin{array}{l}88 \\
88 \\
78 \\
90 \\
88\end{array}$ & $\begin{array}{r}93 \\
93 \\
85 \\
110 \\
104\end{array}$ & $\begin{array}{l}-- \\
-- \\
-- \\
-- \\
--\end{array}$ & $\begin{array}{l}27.2 \\
28.5 \\
28.6 \\
30.8 \\
32.1\end{array}$ & $\begin{array}{l}56.7 \\
58.4 \\
59.0 \\
59.4 \\
60.4\end{array}$ & $\begin{array}{r}2.4 \\
3.3 \\
4.6 \\
7.4 \\
10.3\end{array}$ \\
\hline $\begin{array}{l}26 \ldots \ldots \ldots \\
27 \ldots \ldots \ldots \\
28 \ldots \ldots \ldots \\
29 \ldots \ldots \ldots \\
30 \ldots \ldots \ldots\end{array}$ & $\begin{array}{l}-- \\
-- \\
-- \\
-- \\
--\end{array}$ & $\begin{array}{l}416 \\
402 \\
412 \\
415 \\
--\end{array}$ & $\begin{array}{l}294 \\
228 \\
268 \\
311 \\
--\end{array}$ & $\begin{array}{l}87 \\
67 \\
78 \\
93 \\
--\end{array}$ & $\begin{array}{r}101 \\
66 \\
94 \\
113 \\
--\end{array}$ & $\begin{array}{l}-- \\
-- \\
-- \\
-- \\
--\end{array}$ & $\begin{array}{c}30.4 \\
28.1 \\
29.7 \\
30.4 \\
--\end{array}$ & $\begin{array}{c}57.6 \\
51.3 \\
56.3 \\
56.7 \\
\ldots\end{array}$ & $\begin{array}{l}8.5 \\
8.8 \\
8.8 \\
9.0 \\
--\end{array}$ \\
\hline Mean..... & - & $\overline{395}$ & $\overline{293}$ & $\overline{83}$ & 94 & $\overline{23.4}$ & $\overline{28.3}$ & $\overline{56.4}$ & 6.5 \\
\hline
\end{tabular}


Table 3. Radiation and temperature field data for crater 3, June 1985 through June 1986 [Short-wave radiation measured by pyranometer. In, incoming radiation; Out, outgoing radiation. --, no data or not calculated]

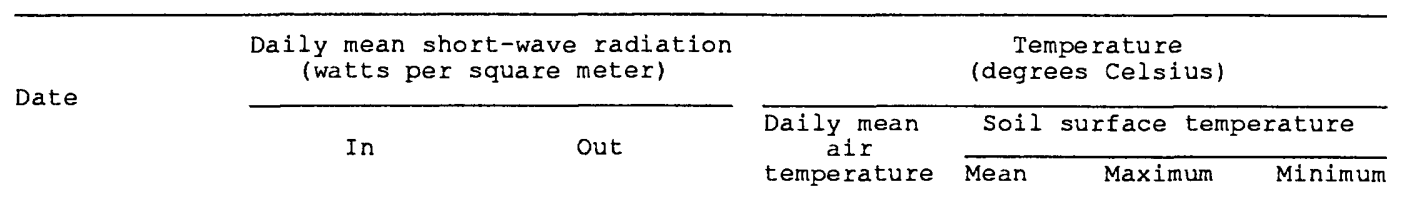

June 1985

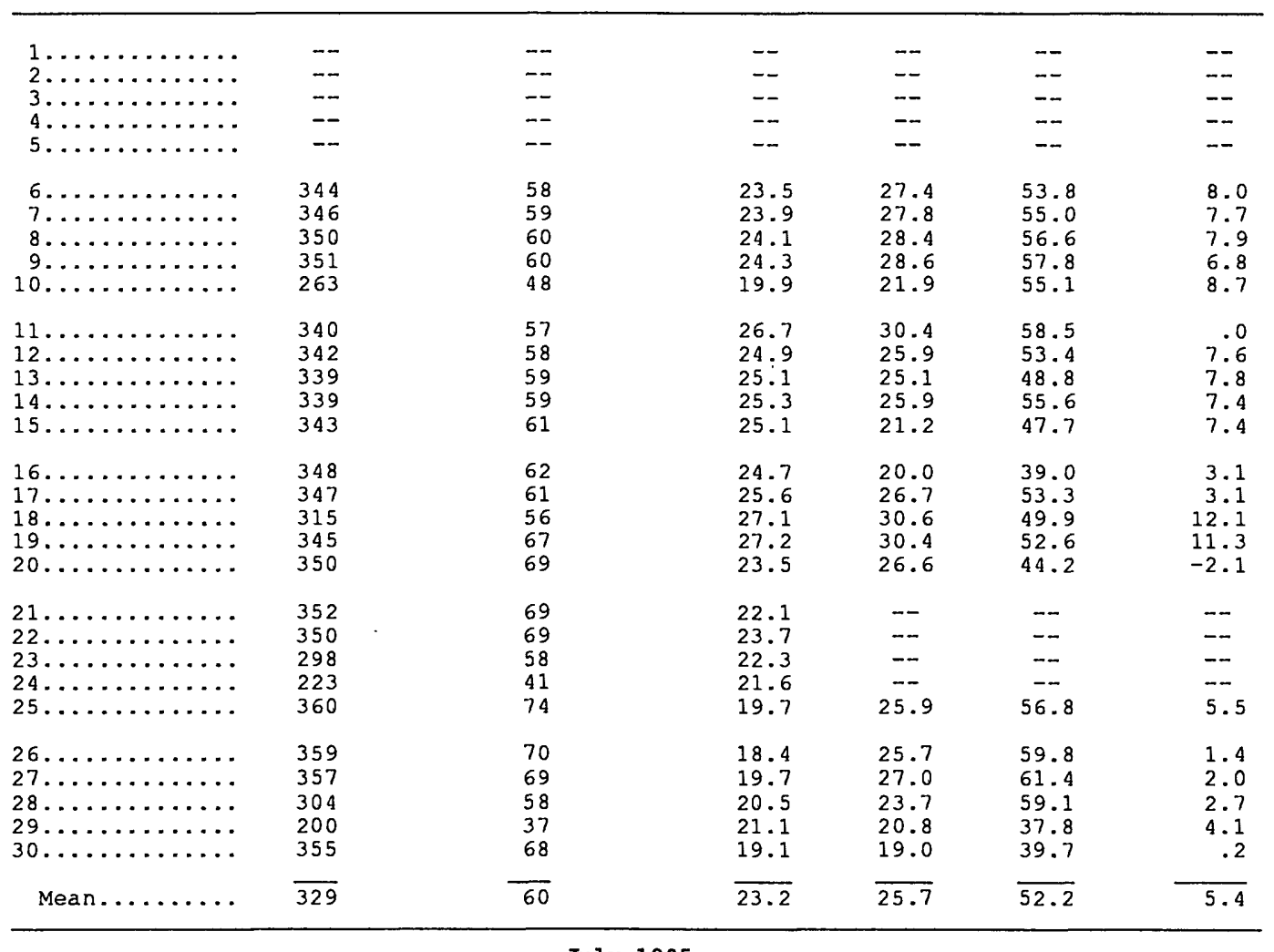

July 1985

\begin{tabular}{|c|c|c|c|c|c|c|}
\hline $\begin{array}{l}1 \ldots \ldots \ldots \\
2 \ldots \ldots \ldots \\
3 \ldots \ldots \ldots \\
4 \ldots \ldots \ldots \\
5 \ldots \ldots \ldots\end{array}$ & $\begin{array}{l}294 \\
353 \\
353 \\
346 \\
329\end{array}$ & $\begin{array}{l}58 \\
70 \\
70 \\
70 \\
66\end{array}$ & $\begin{array}{l}18.4 \\
23.3 \\
24.9 \\
26.4 \\
27.5\end{array}$ & $\begin{array}{l}21.1 \\
28.8 \\
30.1 \\
30.9 \\
32.0\end{array}$ & $\begin{array}{l}60.3 \\
63 \cdot 1 \\
64 \cdot 5 \\
61 \cdot 0 \\
62.3\end{array}$ & $\begin{array}{r}2.0 \\
3.9 \\
5.2 \\
8.0 \\
10.3\end{array}$ \\
\hline $\begin{array}{r}6 \ldots \ldots \ldots \\
7 \ldots \ldots \ldots \\
8 \ldots \ldots \ldots \ldots \\
9 \ldots \ldots \ldots \ldots \\
10 \ldots \ldots \ldots \ldots\end{array}$ & $\begin{array}{l}284 \\
287 \\
287 \\
301 \\
299\end{array}$ & $\begin{array}{l}55 \\
54 \\
54 \\
60 \\
58\end{array}$ & $\begin{array}{l}29.6 \\
26.5 \\
27.1 \\
31.4 \\
31.2\end{array}$ & $\begin{array}{l}33.2 \\
25.9 \\
25.2 \\
33.4 \\
33.7\end{array}$ & $\begin{array}{l}62.4 \\
48.0 \\
47.1 \\
46.7 \\
45.4\end{array}$ & $\begin{array}{r}16.2 \\
12.4 \\
2.3 \\
19.3 \\
20.5\end{array}$ \\
\hline $\begin{array}{l}11 \ldots \ldots \ldots \\
12 \ldots \ldots \ldots \\
13 \ldots \ldots \ldots \ldots \\
14 \ldots \ldots \ldots \ldots \\
15 \ldots \ldots \ldots \ldots\end{array}$ & $\begin{array}{l}322 \\
318 \\
334 \\
307 \\
293\end{array}$ & $\begin{array}{l}64 \\
63 \\
67 \\
64 \\
58\end{array}$ & $\begin{array}{l}29.2 \\
28.8 \\
26.5 \\
25.8 \\
26.0\end{array}$ & $\begin{array}{l}32.4 \\
32.1 \\
30.1 \\
29.1 \\
30.2\end{array}$ & $\begin{array}{l}45.8 \\
46.4 \\
49.7 \\
49.4 \\
50.0\end{array}$ & $\begin{array}{l}15.0 \\
17.1 \\
10.4 \\
10.5 \\
10.9\end{array}$ \\
\hline
\end{tabular}


Table 3. Radiation and temperature field data for crater 3, June 1985 through June 1986 -Continued

\begin{tabular}{|c|c|c|c|c|c|c|}
\hline \multirow{3}{*}{ Date } & \multicolumn{2}{|c|}{$\begin{array}{l}\text { Daily mean short-wave radiation } \\
\text { (watts per square meter) }\end{array}$} & \multicolumn{4}{|c|}{$\begin{array}{c}\text { Temperature } \\
\text { (degrees Celsius) }\end{array}$} \\
\hline & \multirow{2}{*}{ In } & \multirow{2}{*}{ Out } & \multirow{2}{*}{$\begin{array}{c}\text { Daily mean } \\
\text { air } \\
\text { temperature }\end{array}$} & \multirow{2}{*}{$\frac{\text { Soil }}{\text { Mean }}$} & surface & temperature \\
\hline & & & & & Maximum & Minimum \\
\hline \multicolumn{7}{|c|}{ July 1985-Continued } \\
\hline $\begin{array}{l}16 \ldots \ldots \ldots \ldots \ldots \\
17 \ldots \ldots \ldots \\
18 \ldots \ldots \ldots \\
19 \ldots \ldots \ldots \ldots \\
20 \ldots \ldots \ldots \ldots\end{array}$ & $\begin{array}{l}194 \\
308 \\
187 \\
234 \\
245\end{array}$ & $\begin{array}{l}34 \\
59 \\
26 \\
42 \\
34\end{array}$ & $\begin{array}{l}24.8 \\
27.0 \\
23.7 \\
22.1 \\
22.4\end{array}$ & $\begin{array}{l}28.1 \\
32.2 \\
23.8 \\
-- \\
--\end{array}$ & $\begin{array}{l}52.3 \\
57.0 \\
38.2 \\
-- \\
--\end{array}$ & $\begin{array}{c}13.1 \\
13.4 \\
-- \\
-- \\
--\end{array}$ \\
\hline $\begin{array}{l}21 \ldots \ldots \ldots \ldots \\
22 \ldots \ldots \ldots \\
23 \ldots \ldots \ldots \ldots \\
24 \ldots \ldots \ldots \ldots \\
25 \ldots \ldots \ldots \ldots\end{array}$ & $\begin{array}{l}263 \\
330 \\
311 \\
313 \\
295\end{array}$ & $\begin{array}{l}38 \\
60 \\
58 \\
59 \\
58\end{array}$ & $\begin{array}{l}22.7 \\
23.3 \\
25.5 \\
26.6 \\
25.0\end{array}$ & $\begin{array}{l}-- \\
21.6 \\
26.9 \\
29.2 \\
27.7\end{array}$ & $\begin{array}{l}-- \\
34.6 \\
38.6 \\
42.9 \\
43.1\end{array}$ & $\begin{array}{r}-1 \\
11.6 \\
13.7 \\
13.4\end{array}$ \\
\hline $\begin{array}{l}26 \ldots \ldots \ldots \ldots \ldots \\
27 \ldots \ldots \ldots \ldots \\
28 \ldots \ldots \ldots \ldots \\
29 \ldots \ldots \ldots \ldots \\
30 \ldots \ldots \ldots \ldots\end{array}$ & $\begin{array}{l}295 \\
298 \\
324 \\
331 \\
332\end{array}$ & $\begin{array}{l}56 \\
56 \\
65 \\
67 \\
67\end{array}$ & $\begin{array}{l}25.0 \\
25.5 \\
24.2 \\
23.6 \\
21.9\end{array}$ & $\begin{array}{l}27.8 \\
28.5 \\
27.0 \\
25.6 \\
24.9\end{array}$ & $\begin{array}{l}44.0 \\
43.7 \\
41.4 \\
37.7 \\
38.1\end{array}$ & $\begin{array}{l}12.3 \\
11.6 \\
12.2 \\
11.0 \\
10.7\end{array}$ \\
\hline $31 \ldots \ldots \ldots$ & 337 & 70 & 21.1 & 23.3 & 38.1 & 5.4 \\
\hline Mean............... & $\overline{300}$ & 57 & $\overline{25.4}$ & $\overline{28.4}$ & $\overline{48.3}$ & 10.8 \\
\hline \multicolumn{7}{|c|}{ August 1985} \\
\hline $\begin{array}{l}1 \ldots \ldots \ldots \\
2 \ldots \ldots \ldots \\
3 \ldots \ldots \ldots \\
4 \ldots \ldots \\
5 \ldots \ldots\end{array}$ & $\begin{array}{l}335 \\
331 \\
329 \\
330 \\
330\end{array}$ & $\begin{array}{l}70 \\
68 \\
67 \\
68 \\
68\end{array}$ & $\begin{array}{l}20.6 \\
20.2 \\
20.7 \\
21.6 \\
21.3\end{array}$ & $\begin{array}{l}22.8 \\
23.6 \\
24.0 \\
24.6 \\
26.3\end{array}$ & $\begin{array}{l}37.2 \\
39.3 \\
42.4 \\
41.9 \\
47.7\end{array}$ & $\begin{array}{l}5.6 \\
5.7 \\
3.8 \\
4.4 \\
4.3\end{array}$ \\
\hline $\begin{array}{c}6 \ldots \ldots \ldots \ldots \\
7 \ldots \ldots \ldots \\
8 \ldots \ldots \ldots \\
9 \ldots \ldots \ldots \ldots \\
10 \ldots \ldots \ldots \ldots \\
\ldots \ldots \ldots \ldots\end{array}$ & $\begin{array}{l}328 \\
307 \\
309 \\
321 \\
319\end{array}$ & $\begin{array}{l}70 \\
63 \\
63 \\
64 \\
65\end{array}$ & $\begin{array}{l}21.3 \\
22.1 \\
22.1 \\
22.9 \\
22.9\end{array}$ & $\begin{array}{l}26.6 \\
26.4 \\
26.4 \\
28.2 \\
27.1\end{array}$ & $\begin{array}{l}50.1 \\
45.6 \\
44.7 \\
50.0 \\
45.7\end{array}$ & $\begin{array}{l}4.4 \\
5.9 \\
7.4 \\
7.9 \\
6.0\end{array}$ \\
\hline $\begin{array}{l}11 \ldots \ldots \ldots \\
12 \ldots \ldots \ldots \\
13 \ldots \ldots \ldots \\
14 \ldots \ldots \ldots \ldots \\
15 \ldots \ldots \ldots \ldots\end{array}$ & $\begin{array}{l}308 \\
313 \\
314 \\
284 \\
306\end{array}$ & $\begin{array}{l}61 \\
63 \\
64 \\
57 \\
63\end{array}$ & $\begin{array}{l}23 \cdot 1 \\
24.2 \\
20.5 \\
20.6 \\
21.7\end{array}$ & $\begin{array}{l}27.6 \\
28.9 \\
25.5 \\
23.2 \\
24.2\end{array}$ & $\begin{array}{l}48.9 \\
47.1 \\
47.6 \\
46.9 \\
46.6\end{array}$ & $\begin{array}{r}7.4 \\
11.2 \\
4.4 \\
3.1 \\
5.0\end{array}$ \\
\hline $\begin{array}{l}16 \ldots \ldots \ldots \\
17 \ldots \ldots \ldots \\
18 \ldots \ldots \ldots \\
19 \ldots \ldots \ldots \\
20 \ldots \ldots \ldots\end{array}$ & $\begin{array}{l}291 \\
274 \\
315 \\
258 \\
314\end{array}$ & $\begin{array}{l}58 \\
55 \\
68 \\
57 \\
68\end{array}$ & $\begin{array}{l}22.5 \\
22.0 \\
19.7 \\
19.7 \\
21.4\end{array}$ & $\begin{array}{l}23.8 \\
29.8 \\
25.9 \\
22.5 \\
24.1\end{array}$ & $\begin{array}{l}41.4 \\
41.6 \\
41.0 \\
39.3 \\
40.2\end{array}$ & $\begin{array}{r}7.1 \\
16.0 \\
8.5 \\
5.9 \\
7.5\end{array}$ \\
\hline $\begin{array}{l}21 \ldots \ldots \ldots \ldots \\
22 \ldots \ldots \ldots \\
23 \ldots \ldots \ldots \ldots \\
24 \ldots \ldots \ldots \ldots \\
25 \ldots \ldots \ldots \ldots\end{array}$ & $\begin{array}{l}272 \\
288 \\
295 \\
294 \\
288\end{array}$ & $\begin{array}{l}56 \\
58 \\
60 \\
60 \\
60\end{array}$ & $\begin{array}{l}20.2 \\
20.0 \\
22.0 \\
23.8 \\
24.5\end{array}$ & $\begin{array}{l}22.5 \\
22.6 \\
24.8 \\
26.4 \\
27.0\end{array}$ & $\begin{array}{l}38.4 \\
42.6 \\
45.8 \\
48.2 \\
46.8\end{array}$ & $\begin{array}{l}6.6 \\
4.5 \\
5.0 \\
6.0 \\
8.3\end{array}$ \\
\hline $\begin{array}{l}26 \ldots \ldots \ldots \ldots \\
27 \ldots \ldots \ldots \\
28 \ldots \ldots \ldots \ldots \\
29 \ldots \ldots \ldots \ldots \\
30 \ldots \ldots \ldots \ldots\end{array}$ & $\begin{array}{l}280 \\
290 \\
294 \\
287 \\
278\end{array}$ & $\begin{array}{l}57 \\
61 \\
62 \\
60 \\
58\end{array}$ & $\begin{array}{l}25.8 \\
24.1 \\
21.7 \\
21.4 \\
23.1\end{array}$ & $\begin{array}{l}29.4 \\
28.2 \\
25.8 \\
25.8 \\
26.7\end{array}$ & $\begin{array}{l}47.0 \\
46.4 \\
46.5 \\
48.0 \\
46.9\end{array}$ & $\begin{array}{r}13.3 \\
12.0 \\
7.5 \\
5.0 \\
7.2\end{array}$ \\
\hline $31 \ldots \ldots \ldots$ & 283 & 58 & 23.7 & 27.7 & 47.0 & 10.3 \\
\hline 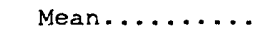 & $\overline{302}$ & 62 & $\overline{22.0}$ & $\overline{25.7}$ & $\overline{44.8}$ & 7.0 \\
\hline
\end{tabular}


Table 3. Radiation and temperature field data for crater 3, June 1985 through June 1986 -Continued

\begin{tabular}{|c|c|c|c|c|c|c|}
\hline \multirow{3}{*}{ Date } & \multicolumn{2}{|c|}{$\begin{array}{l}\text { Daily mean short-wave radiation } \\
\text { (watts per square meter) }\end{array}$} & \multicolumn{4}{|c|}{$\begin{array}{l}\text { Temperature } \\
\text { (degrees Celsius) }\end{array}$} \\
\hline & \multirow{2}{*}{ In } & \multirow{2}{*}{ Out } & \multirow{2}{*}{$\begin{array}{c}\text { Daily mean } \\
\text { air } \\
\text { temperature }\end{array}$} & \multicolumn{2}{|c|}{ Soil surface $t$} & temperature \\
\hline & & & & Mean & Maximum & $\overline{\text { Minimum }}$ \\
\hline \multicolumn{7}{|c|}{ Septamber 1985} \\
\hline $\begin{array}{l}1 \ldots \ldots \ldots \\
2 \ldots \ldots \ldots \\
{ }_{2} \ldots \ldots \\
5 \ldots \ldots\end{array}$ & $\begin{array}{l}278 \\
282 \\
284 \\
221 \\
190\end{array}$ & $\begin{array}{l}57 \\
60 \\
53 \\
41 \\
33\end{array}$ & $\begin{array}{l}23.9 \\
24.1 \\
20.0 \\
14.7 \\
14.7\end{array}$ & $\begin{array}{l}26.9 \\
27.2 \\
23.6 \\
18.2 \\
18.2\end{array}$ & $\begin{array}{l}43.0 \\
41.0 \\
40.1 \\
35.9 \\
36.8\end{array}$ & $\begin{array}{r}10.2 \\
11.3 \\
7.9 \\
3.0 \\
4.4\end{array}$ \\
\hline $\begin{array}{r}6 \ldots \ldots \cdots \cdots \\
7 \ldots \ldots \cdots \cdots \\
8 \ldots \ldots \cdots \cdots \\
9 \ldots \ldots \cdots \cdots\end{array}$ & $\begin{array}{l}187 \\
254 \\
232 \\
272 \\
267\end{array}$ & $\begin{array}{l}33 \\
47 \\
41 \\
52 \\
52\end{array}$ & $\begin{array}{l}12.5 \\
15.0 \\
17.1 \\
18.3 \\
13.7\end{array}$ & $\begin{array}{l}15.7 \\
18.4 \\
20.0 \\
20.6 \\
17.0\end{array}$ & $\begin{array}{l}34.9 \\
35.8 \\
35.4 \\
31.8 \\
33.0\end{array}$ & $\begin{array}{l}2.1 \\
.8 \\
6.4 \\
9.7 \\
1.0\end{array}$ \\
\hline $\begin{array}{l}11 \ldots \ldots \cdots \cdots \\
12 \ldots \ldots \cdots \cdots \\
13 \ldots \ldots \cdots \cdots \\
14 \ldots \ldots \cdots \cdots\end{array}$ & $\begin{array}{l}197 \\
264 \\
254 \\
254 \\
250\end{array}$ & $\begin{array}{l}38 \\
50 \\
48 \\
50 \\
49\end{array}$ & $\begin{array}{l}10.4 \\
11.2 \\
13.5 \\
16.3 \\
12.6\end{array}$ & $\begin{array}{l}12.8 \\
15.7 \\
17.3 \\
19.9 \\
19.8\end{array}$ & $\begin{array}{l}26.9 \\
39.0 \\
40.2 \\
39.8 \\
39.1\end{array}$ & $\begin{array}{r}1.0 \\
-3.6 \\
-.9 \\
1.8 \\
4.2\end{array}$ \\
\hline 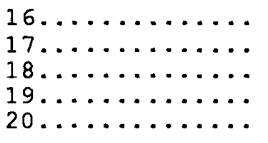 & $\begin{array}{r}255 \\
252 \\
65 \\
172 \\
242\end{array}$ & $\begin{array}{r}49 \\
52 \\
8 \\
25 \\
44\end{array}$ & $\begin{array}{l}15.1 \\
15.6 \\
14.9 \\
13.9 \\
12.1\end{array}$ & $\begin{array}{l}18.9 \\
18.1 \\
16.6 \\
16.7 \\
16.0\end{array}$ & $\begin{array}{l}38.0 \\
33.7 \\
24.1 \\
30.0 \\
37.5\end{array}$ & $\begin{array}{r}1.9 \\
.5 \\
11.1 \\
4.9 \\
.9\end{array}$ \\
\hline $\begin{array}{l}21 \ldots \ldots \cdots \cdots \\
22 \ldots \ldots \cdots \cdots \\
23 \ldots \ldots \cdots \cdots \\
24 \ldots \ldots \cdots \cdots \\
25 \ldots \ldots \cdots \cdots\end{array}$ & $\begin{array}{l}246 \\
244 \\
236 \\
238 \\
236\end{array}$ & $\begin{array}{l}45 \\
45 \\
43 \\
44 \\
43\end{array}$ & $\begin{array}{l}12.2 \\
11.4 \\
12.3 \\
16.7 \\
16.8\end{array}$ & $\begin{array}{l}16.4 \\
17.3 \\
18.4 \\
20.5 \\
20.5\end{array}$ & $\begin{array}{l}38.6 \\
37.3 \\
42.7 \\
44.9 \\
43.4\end{array}$ & $\begin{array}{r}-2.8 \\
1.0 \\
-1.0 \\
1.2 \\
1.8\end{array}$ \\
\hline 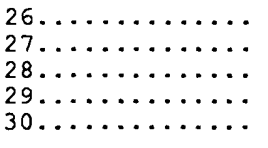 & $\begin{array}{l}231 \\
155 \\
221 \\
221 \\
215\end{array}$ & $\begin{array}{l}43 \\
25 \\
39 \\
40 \\
41\end{array}$ & $\begin{array}{l}15.4 \\
14.7 \\
16.9 \\
11.5 \\
13.9\end{array}$ & $\begin{array}{l}19.7 \\
19.4 \\
20.4 \\
15.6 \\
17.4\end{array}$ & $\begin{array}{l}42.1 \\
36.2 \\
39.4 \\
37.5 \\
36.6\end{array}$ & $\begin{array}{r}1.6 \\
6.4 \\
4.5 \\
-3.5 \\
1.0\end{array}$ \\
\hline 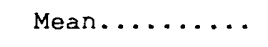 & $\overline{230}$ & $\overline{43}$ & $\overline{15.0}$ & $\overline{18.8}$ & $\overline{37.2}$ & $\overline{3.0}$ \\
\hline \multicolumn{7}{|c|}{ October 1985} \\
\hline $\begin{array}{l}1 \ldots \ldots \cdots \cdots \\
2 \ldots \ldots \cdots \cdots \\
3 \ldots \ldots \cdots \cdots \\
5 \ldots \ldots \cdots \cdots\end{array}$ & $\begin{array}{l}201 \\
218 \\
218 \\
210 \\
217\end{array}$ & $\begin{array}{l}38 \\
43 \\
42 \\
41 \\
42\end{array}$ & $\begin{array}{l}15.4 \\
13.4 \\
15.8 \\
18.0 \\
14.6\end{array}$ & $\begin{array}{l}19.4 \\
16.8 \\
19.2 \\
21.8 \\
18.9\end{array}$ & $\begin{array}{l}40.7 \\
39.0 \\
42.7 \\
43.8 \\
41.6\end{array}$ & $\begin{array}{r}2.3 \\
-2.2 \\
1.2 \\
4.8 \\
.0\end{array}$ \\
\hline $\begin{array}{c}6 \ldots \ldots \cdots \cdots \cdots \\
7 \ldots \ldots \cdots \cdots \\
8 \ldots \ldots \cdots \cdots \\
9 \ldots \ldots \cdots \cdots\end{array}$ & $\begin{array}{r}205 \\
201 \\
69 \\
103 \\
122\end{array}$ & $\begin{array}{r}40 \\
41 \\
5 \\
13 \\
16\end{array}$ & $\begin{array}{r}17.7 \\
15.6 \\
9.2 \\
7.5 \\
10.2\end{array}$ & $\begin{array}{r}20.4 \\
17.7 \\
11.6 \\
9.3 \\
12.3\end{array}$ & $\begin{array}{l}36.6 \\
31.2 \\
20.9 \\
22.2 \\
25.4\end{array}$ & $\begin{array}{r}6.6 \\
2.6 \\
2.8 \\
1.6 \\
.6\end{array}$ \\
\hline 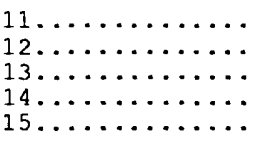 & $\begin{array}{l}205 \\
181 \\
201 \\
204 \\
201\end{array}$ & $\begin{array}{l}36 \\
33 \\
37 \\
38 \\
39\end{array}$ & $\begin{array}{r}7.6 \\
8.2 \\
12.1 \\
9.0 \\
8.6\end{array}$ & $\begin{array}{l}11.0 \\
11.6 \\
14.4 \\
11.4 \\
11.9\end{array}$ & $\begin{array}{l}30.0 \\
31.2 \\
28.0 \\
29.5 \\
32.7\end{array}$ & $\begin{array}{r}-3.3 \\
-4.5 \\
1.4 \\
-4.0 \\
-4.4\end{array}$ \\
\hline 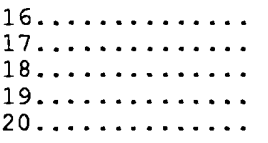 & $\begin{array}{l}195 \\
190 \\
188 \\
183 \\
145\end{array}$ & $\begin{array}{l}35 \\
34 \\
34 \\
33 \\
26\end{array}$ & $\begin{array}{r}5.1 \\
7.3 \\
8.3 \\
8.3 \\
11.1\end{array}$ & $\begin{array}{l}11.7 \\
12.6 \\
12.5 \\
11.9 \\
13.6\end{array}$ & $\begin{array}{l}38.7 \\
38.6 \\
35.2 \\
34.7 \\
32.5\end{array}$ & $\begin{array}{r}-6.1 \\
-5.9 \\
-4.9 \\
-4.8 \\
-.3\end{array}$ \\
\hline
\end{tabular}


Table 3. Radiation and temperature field data for crater 3, June 1985 through June 1986 - Continued

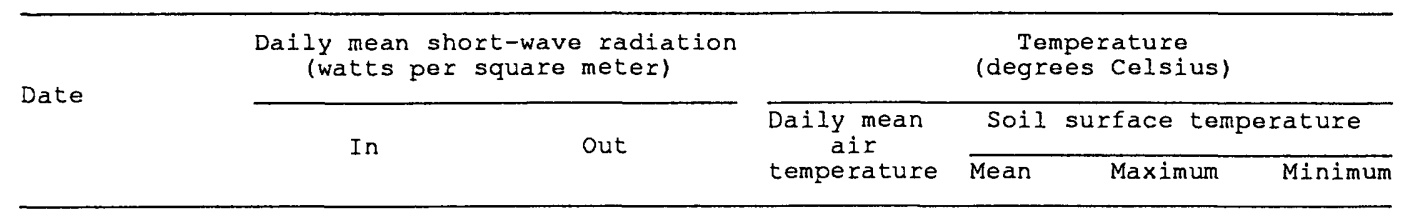

October 1985-Continued

\begin{tabular}{|c|c|c|c|c|c|c|}
\hline $\begin{array}{l}21 \ldots \ldots \ldots \ldots \\
22 \ldots \ldots \ldots \ldots \ldots \\
23 \ldots \ldots \ldots \ldots \ldots \\
24 \ldots \ldots \ldots \ldots \\
25 \ldots \ldots \ldots \ldots \ldots\end{array}$ & $\begin{array}{r}-- \\
-- \\
174 \\
172 \\
171\end{array}$ & $\begin{array}{l}-- \\
-- \\
32 \\
31 \\
32\end{array}$ & $\begin{array}{l}-- \\
-- \\
6.5 \\
9.2 \\
7.9\end{array}$ & $\begin{array}{c}-- \\
-- \\
10.2 \\
12.9 \\
12.7\end{array}$ & $\begin{array}{l}-- \\
-\overline{-} \\
33.3 \\
37.3 \\
36.3\end{array}$ & $\begin{array}{l}-- \\
-- \\
-6.2 \\
-4.1 \\
-4.0\end{array}$ \\
\hline $\begin{array}{l}26 \ldots \ldots \ldots \ldots \\
27 \ldots \ldots \ldots \ldots \\
28 \ldots \ldots \ldots \ldots \\
29 \ldots \ldots \ldots \ldots \\
30 \ldots \ldots \ldots \ldots\end{array}$ & $\begin{array}{r}170 \\
167 \\
84 \\
163 \\
148\end{array}$ & $\begin{array}{l}31 \\
31 \\
14 \\
36 \\
34\end{array}$ & $\begin{array}{l}9.2 \\
-- \\
-- \\
-- \\
6.3\end{array}$ & $\begin{array}{l}13.1 \\
12.7 \\
11.2 \\
12.2 \\
11.4\end{array}$ & $\begin{array}{l}38.8 \\
37.7 \\
36.1 \\
36.8 \\
33.3\end{array}$ & $\begin{array}{l}-3 \cdot 6 \\
-4 \cdot 3 \\
-.3 \\
-4.0 \\
-5 \cdot 1\end{array}$ \\
\hline $31 \ldots \ldots \ldots$ & 180 & 42 & 13.6 & 14.7 & 24.8 & 6.8 \\
\hline \multirow[t]{2}{*}{ Mean........ } & $\overline{175}$ & 33 & $\overline{10.6}$ & $\overline{13.8}$ & $\overline{34.1}$ & $-1 \cdot 4$ \\
\hline & \multicolumn{3}{|c|}{ November 1985} & . & & \\
\hline $\begin{array}{l}1 \ldots \ldots \ldots \\
2 \ldots \ldots \ldots \\
3 \ldots \ldots \ldots \\
4 \ldots \ldots \ldots \\
5 \ldots \ldots \ldots\end{array}$ & $\begin{array}{l}-- \\
-- \\
-- \\
185\end{array}$ & $\begin{array}{l}-\overline{-} \\
-- \\
-- \\
\overline{42}\end{array}$ & $\begin{array}{l}-- \\
-- \\
-- \\
-- \\
12.4\end{array}$ & $\begin{array}{l}-- \\
-- \\
-- \\
-- \\
14.9\end{array}$ & $\begin{array}{c}-- \\
-- \\
-- \\
-- \\
31.2\end{array}$ & $\begin{array}{l}-- \\
-- \\
-- \\
-- \\
-1.5\end{array}$ \\
\hline $\begin{array}{r}6 \ldots \ldots \ldots \ldots \\
7 \ldots \ldots \ldots \\
8 \ldots \ldots \ldots \ldots \\
9 \ldots \ldots \ldots \ldots \\
10 \ldots \ldots \ldots \ldots\end{array}$ & $\begin{array}{l}142 \\
133 \\
143 \\
130\end{array}$ & $\begin{array}{l}-\overline{31} \\
29 \\
31 \\
26\end{array}$ & $\begin{array}{l}7.9 \\
5.9 \\
5.0 \\
8.5\end{array}$ & $\begin{array}{l}-- \\
9.9 \\
8.6 \\
7.0 \\
9.5\end{array}$ & $\begin{array}{l}3 \overline{3} .2 \\
28.8 \\
26.0 \\
17.0\end{array}$ & $\begin{array}{r}-- \\
-5.3 \\
-4.6 \\
-7.2 \\
4.0\end{array}$ \\
\hline $\begin{array}{l}11 \ldots \ldots \ldots \\
12 \ldots \ldots \ldots \\
13 \ldots \ldots \ldots \\
14 \ldots \ldots \ldots \\
15 \ldots \ldots \ldots \ldots\end{array}$ & $\begin{array}{r}-- \\
-- \\
154 \\
149 \\
148\end{array}$ & $\begin{array}{l}-- \\
57 \\
40 \\
27 \\
22\end{array}$ & $\begin{array}{r}-- \\
-.9 \\
-3.5 \\
-1.1 \\
-1.1\end{array}$ & $\begin{array}{r}-- \\
1.2 \\
-1.2 \\
.4 \\
2.7\end{array}$ & $\begin{array}{l}-\overline{1} \\
12.6 \\
19.3 \\
12.5 \\
27.7\end{array}$ & $\begin{array}{r}-\overline{-} \\
-8.2 \\
-12.0 \\
-7.4 \\
-9.0\end{array}$ \\
\hline $\begin{array}{l}16 \ldots \ldots \ldots \\
17 \ldots \ldots \ldots \\
18 \ldots \ldots \ldots \\
19 \ldots \ldots \ldots \\
20 \ldots \ldots \ldots \ldots\end{array}$ & $\begin{array}{l}113 \\
143 \\
140 \\
138 \\
128\end{array}$ & $\begin{array}{l}17 \\
21 \\
26 \\
25 \\
22\end{array}$ & $\begin{array}{l}-.4 \\
-- \\
-.4 \\
-3.8 \\
--\end{array}$ & $\begin{array}{r}2.9 \\
4.2 \\
.9 \\
-1.6 \\
-1.3\end{array}$ & $\begin{array}{r}21.3 \\
21.1 \\
8.1 \\
15.2 \\
20.2\end{array}$ & $\begin{array}{r}-7.6 \\
-7.4 \\
-6.9 \\
-11.4 \\
-14.3\end{array}$ \\
\hline $\begin{array}{l}21 \ldots \ldots \ldots \\
22 \ldots \ldots \ldots \\
23 \ldots \ldots \ldots \\
24 \ldots \ldots \ldots \\
25 \ldots \ldots \ldots \ldots\end{array}$ & $\begin{array}{r}141 \\
122 \\
55 \\
64 \\
21\end{array}$ & $\begin{array}{r}25 \\
22 \\
8 \\
9 \\
0\end{array}$ & $\begin{array}{r}-2.8 \\
-2.3 \\
1.1 \\
4.9 \\
6.0\end{array}$ & $\begin{array}{r}.7 \\
.4 \\
3.2 \\
6.5 \\
8.0\end{array}$ & $\begin{array}{l}19.3 \\
19.1 \\
19.7 \\
19.8 \\
13.7\end{array}$ & $\begin{array}{r}-10.3 \\
-9.8 \\
-7.2 \\
-2.0 \\
4.6\end{array}$ \\
\hline $\begin{array}{l}26 \ldots \ldots \ldots \ldots \\
27 \ldots \ldots \ldots \\
28 \ldots \ldots \ldots \\
29 \ldots \ldots \ldots \ldots \\
30 \ldots \ldots \ldots \ldots\end{array}$ & $\begin{array}{r}129 \\
99 \\
69 \\
55 \\
119\end{array}$ & $\begin{array}{r}14 \\
12 \\
7 \\
7 \\
14\end{array}$ & $\begin{array}{l}3.0 \\
2.8 \\
2.8 \\
4.4 \\
3.5\end{array}$ & $\begin{array}{l}5.0 \\
3.9 \\
5.2 \\
5.7 \\
5.5\end{array}$ & $\begin{array}{c}-- \\
16.5 \\
19.3 \\
16.9 \\
19.8\end{array}$ & $\begin{array}{r}-5.3 \\
.0 \\
-2.0 \\
.1 \\
-3.5\end{array}$ \\
\hline Mean........ & $\overline{118}$ & 22 & 2.3 & 4.2 & $\overline{19.9}$ & -5.6 \\
\hline
\end{tabular}


Table 3. Radiation and temperature field data for crater 3, June 1985 through June 1986 -Continued

\begin{tabular}{|c|c|c|c|c|c|c|}
\hline \multirow{3}{*}{ Date } & \multicolumn{2}{|c|}{$\begin{array}{l}\text { Daily mean short-wave radiation } \\
\text { (watts per square meter) }\end{array}$} & \multicolumn{4}{|c|}{$\begin{array}{l}\text { Temperature } \\
\text { (degrees Celsius) }\end{array}$} \\
\hline & \multirow{2}{*}{ In } & \multirow{2}{*}{ Out } & \multirow{2}{*}{$\begin{array}{c}\text { Daily mean } \\
\text { air } \\
\text { temperature }\end{array}$} & \multicolumn{2}{|c|}{ Soil surface } & temperature \\
\hline & & & & Mean & Maximum & Minimum \\
\hline \multicolumn{7}{|c|}{ December 1985} \\
\hline $\begin{array}{l}1 \ldots \ldots \ldots \\
2 \ldots \ldots \ldots \\
3 \ldots \ldots \ldots \ldots \\
4 \ldots \ldots \ldots \\
5 \ldots \ldots \ldots \ldots\end{array}$ & $\begin{array}{r}86 \\
-- \\
57 \\
119 \\
85\end{array}$ & $\begin{array}{r}10 \\
-\frac{4}{4} \\
12 \\
9\end{array}$ & $\begin{array}{l}2.0 \\
4.6 \\
5.3 \\
2.2 \\
2.3\end{array}$ & $\begin{array}{l}4.5 \\
6.0 \\
7.9 \\
5.7 \\
4.9\end{array}$ & $\begin{array}{r}19.0 \\
7.8 \\
20.7 \\
26.4 \\
22.5\end{array}$ & $\begin{array}{r}-4.9 \\
.2 \\
-1.8 \\
-5.5 \\
-3.2\end{array}$ \\
\hline $\begin{array}{r}6 \ldots \ldots \ldots \\
7 \ldots \ldots \ldots \\
8 \ldots \ldots \ldots \ldots \\
9 \ldots \ldots \ldots \ldots \ldots \\
10 \ldots \ldots \ldots \ldots\end{array}$ & $\begin{array}{r}116 \\
103 \\
118 \\
-- \\
--\end{array}$ & $\begin{array}{l}15 \\
16 \\
24 \\
-- \\
--\end{array}$ & $\begin{array}{r}.8 \\
.5 \\
2.3 \\
-- \\
--\end{array}$ & $\begin{array}{l}4.7 \\
3.9 \\
3.7 \\
-- \\
--\end{array}$ & $\begin{array}{l}25.2 \\
21.3 \\
13.1 \\
-- \\
--\end{array}$ & $\begin{array}{l}-5.7 \\
-5.2 \\
-9.2 \\
-- \\
--\end{array}$ \\
\hline $\begin{array}{l}11 \ldots \ldots \ldots \ldots \\
12 \ldots \ldots \ldots \\
13 \ldots \ldots \ldots \\
14 \ldots \ldots \ldots \ldots \\
15 \ldots \ldots \ldots \ldots \ldots\end{array}$ & $\begin{array}{l}-- \\
110 \\
110 \\
104\end{array}$ & $\begin{array}{l}-- \\
-- \\
23 \\
22 \\
19\end{array}$ & $\begin{array}{l}-- \\
-- \\
-- \\
-.7 \\
.2\end{array}$ & $\begin{array}{r}-- \\
-- \\
-1.0 \\
1.2 \\
3.5\end{array}$ & $\begin{array}{l}-- \\
-- \\
18.0 \\
23.9 \\
26.7\end{array}$ & $\begin{array}{r}-- \\
-- \\
-12.7 \\
-11.8 \\
-10.9\end{array}$ \\
\hline $\begin{array}{l}16 \ldots \ldots \ldots \ldots \\
17 \ldots \ldots \ldots \\
18 \ldots \ldots \ldots \\
19 \ldots \ldots \ldots \ldots \\
20 \ldots \ldots \ldots \ldots\end{array}$ & 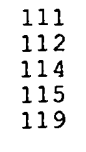 & $\begin{array}{l}23 \\
23 \\
25 \\
24 \\
29\end{array}$ & $\begin{array}{l}3.2 \\
2.5 \\
-- \\
-- \\
--\end{array}$ & $\begin{array}{l}5.4 \\
6.7 \\
8.3 \\
4.9 \\
-.8\end{array}$ & $\begin{array}{l}20.6 \\
21.8 \\
26.4 \\
31.6 \\
29.2\end{array}$ & $\begin{array}{l}-8.3 \\
-8.5 \\
-7.4 \\
-9.2 \\
-9.5\end{array}$ \\
\hline $\begin{array}{l}21 \ldots \ldots \ldots \\
22 \ldots \ldots \ldots \\
23 \ldots \ldots \ldots \\
24 \ldots \ldots \ldots \\
25 \ldots \ldots \ldots\end{array}$ & $\begin{array}{l}-- \\
110 \\
109 \\
105\end{array}$ & $\begin{array}{l}-- \\
-- \\
24 \\
23 \\
22\end{array}$ & $\begin{array}{l}-- \\
-- \\
-- \\
7.2 \\
6.6\end{array}$ & $\begin{array}{l}-- \\
-- \\
4.0 \\
9.0 \\
9.1\end{array}$ & $\begin{array}{l}-- \\
-- \\
26.4 \\
24.4 \\
26.9\end{array}$ & $\begin{array}{r}-- \\
-- \\
-10.0 \\
-4.5 \\
-6.8\end{array}$ \\
\hline $\begin{array}{l}26 \ldots \ldots \ldots \\
27 \ldots \ldots \ldots \\
28 \ldots \ldots \ldots \\
29 \ldots \ldots \ldots \ldots \\
30 \ldots \ldots \ldots\end{array}$ & $\begin{array}{r}110 \\
113 \\
109 \\
49 \\
46\end{array}$ & $\begin{array}{r}22 \\
23 \\
22 \\
7 \\
8\end{array}$ & $\begin{array}{l}-- \\
-- \\
-- \\
-- \\
--\end{array}$ & $\begin{array}{l}3.4 \\
2.8 \\
2.0 \\
5.4 \\
7.8\end{array}$ & $\begin{array}{l}29.8 \\
28.1 \\
26.9 \\
17.5 \\
22.7\end{array}$ & $\begin{array}{l}-10.0 \\
-10.9 \\
-10.8 \\
-6.2 \\
-2.7\end{array}$ \\
\hline $31 \ldots \ldots \ldots$ & 71 & 11 & -- & 4.9 & 24.6 & -5.8 \\
\hline Mean........ & $\overline{100}$ & 18 & 2.8 & 4.7 & $\overline{23.3}$ & -7.2 \\
\hline \multicolumn{7}{|c|}{ January 1986} \\
\hline $\begin{array}{l}1 \ldots \ldots \ldots \ldots \\
2 \ldots \ldots \ldots \ldots \\
3 \ldots \ldots \ldots \ldots \\
4 \ldots \ldots \ldots\end{array}$ & $\begin{array}{r}98 \\
81 \\
114 \\
76 \\
36\end{array}$ & $\begin{array}{r}19 \\
17 \\
24 \\
16 \\
2\end{array}$ & $\begin{array}{l}-- \\
-- \\
-- \\
-- \\
--\end{array}$ & $\begin{array}{l}2.6 \\
3.0 \\
4.7 \\
9.3 \\
8.2\end{array}$ & $\begin{array}{l}24.9 \\
22.7 \\
27.4 \\
22.9 \\
17.6\end{array}$ & $\begin{array}{r}-8.2 \\
-8.7 \\
-8.3 \\
-.5 \\
.0\end{array}$ \\
\hline $\begin{array}{c}6 \ldots \ldots \ldots \ldots \\
7 \ldots \ldots \ldots \\
8 \ldots \ldots \ldots \ldots \\
9 \ldots \ldots \ldots \ldots \ldots \\
10 \ldots \ldots \ldots \ldots\end{array}$ & $\begin{array}{l}112 \\
120 \\
113 \\
141 \\
116\end{array}$ & $\begin{array}{l}22 \\
27 \\
22 \\
29 \\
24\end{array}$ & $\begin{array}{r}-- \\
2.0 \\
-1.2 \\
2.2 \\
2.8\end{array}$ & $\begin{array}{l}6.3 \\
3.7 \\
1.2 \\
4.5 \\
5.5\end{array}$ & $\begin{array}{l}18.6 \\
16.4 \\
25.5 \\
31.3 \\
33.4\end{array}$ & $\begin{array}{r}-2.5 \\
-9.5 \\
-12.4 \\
-10.6 \\
-8.5\end{array}$ \\
\hline $\begin{array}{l}11 \ldots \ldots \ldots \ldots \\
12 \ldots \ldots \ldots \\
13 \ldots \ldots \ldots \\
14 \ldots \ldots \ldots \ldots \\
15 \ldots \ldots \ldots \ldots\end{array}$ & $\begin{array}{r}116 \\
120 \\
-- \\
53 \\
60\end{array}$ & $\begin{array}{l}24 \\
25 \\
-- \\
10 \\
10\end{array}$ & $\begin{array}{l}2.8 \\
2.6 \\
-- \\
2.8 \\
4.9\end{array}$ & $\begin{array}{l}5.7 \\
5.4 \\
-- \\
4.5 \\
7.1\end{array}$ & $\begin{array}{c}32.9 \\
32.2 \\
-- \\
20.4 \\
16.6\end{array}$ & $\begin{array}{l}-8.5 \\
-7.7 \\
-- \\
-6.8 \\
-1.9\end{array}$ \\
\hline $\begin{array}{l}16 \ldots \ldots \ldots \ldots \\
17 \ldots \ldots \ldots \\
18 \ldots \ldots \ldots \ldots \\
19 \ldots \ldots \ldots \ldots \\
20 \ldots \ldots \ldots \ldots\end{array}$ & $\begin{array}{l}87 \\
108 \\
124 \\
107 \\
129\end{array}$ & $\begin{array}{l}17 \\
22 \\
26 \\
24 \\
30\end{array}$ & $\begin{array}{l}1.5 \\
1.9 \\
4.7 \\
3.2 \\
4.7\end{array}$ & $\begin{array}{l}4.1 \\
4.7 \\
7.9 \\
5.8 \\
6.5\end{array}$ & $\begin{array}{l}44.4 \\
26.0 \\
32.4 \\
30.3 \\
23.2\end{array}$ & $\begin{array}{l}-5.8 \\
-6.8 \\
-5.3 \\
-8.0 \\
-4.4\end{array}$ \\
\hline
\end{tabular}


Table 3. Radiation and temperature field data for crater 3, June 1985 through June 1986 -Continued

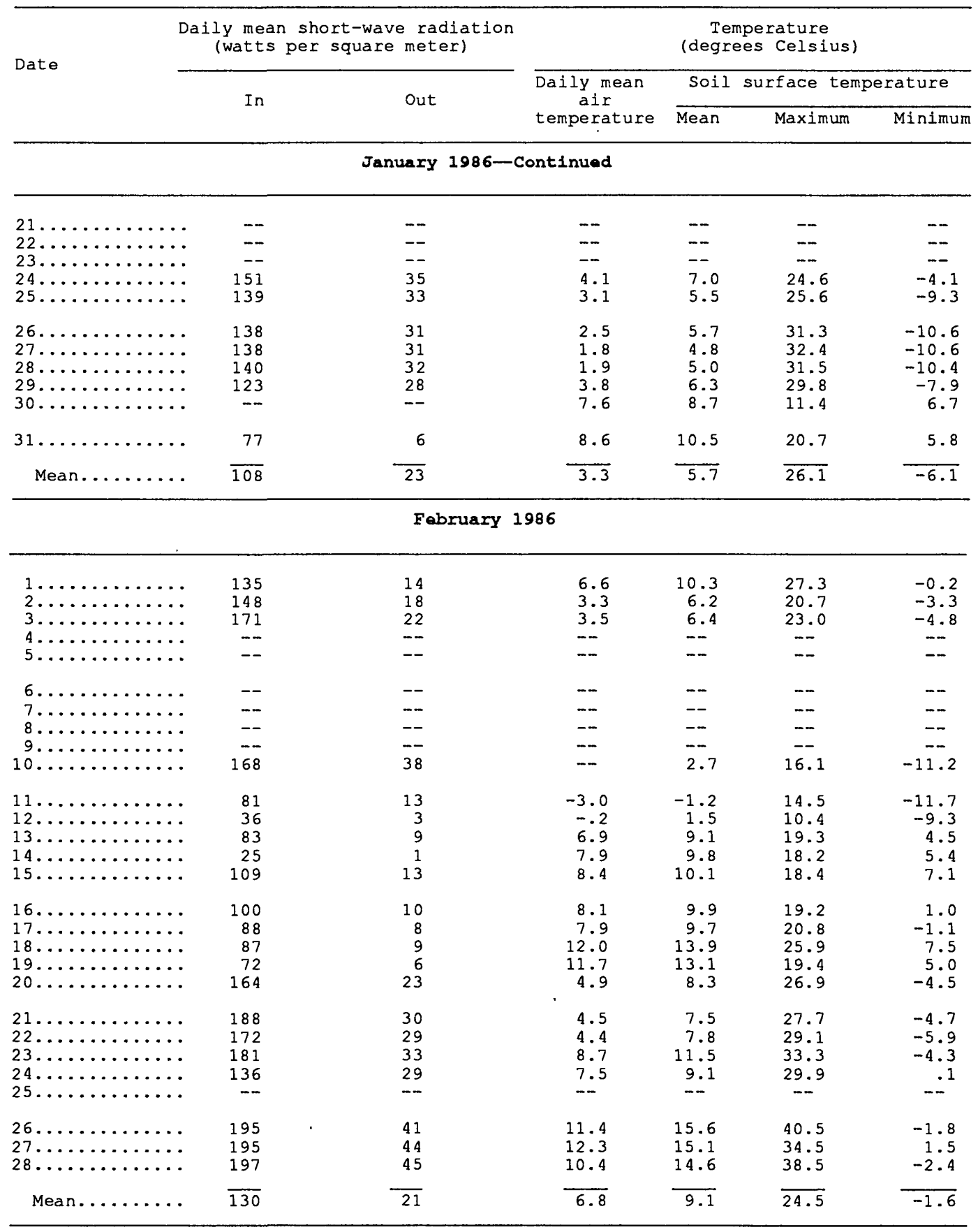


Table 3. Radiation and temperature field data for crater 3, June 1985 through June 1986 -Continued

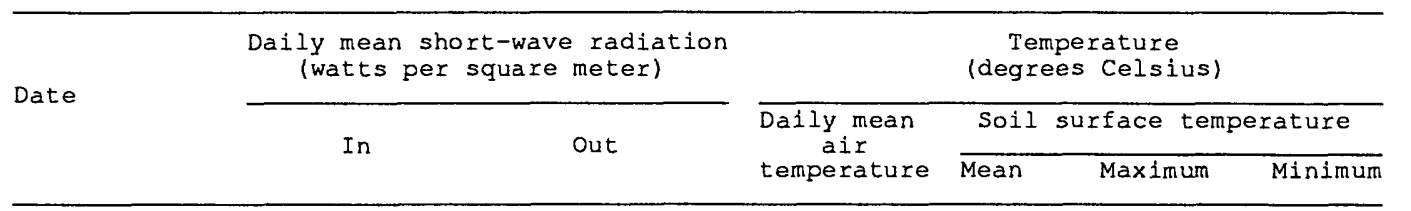

March 1986

\begin{tabular}{|c|c|c|c|c|c|c|}
\hline $\begin{array}{l}1 \ldots \ldots \ldots \\
2 \ldots \ldots \ldots \\
3 \ldots \ldots \ldots \\
4 \ldots \ldots \ldots \\
5 \ldots \ldots \ldots\end{array}$ & $\begin{array}{r}189 \\
82 \\
197 \\
199 \\
209\end{array}$ & $\begin{array}{l}45 \\
15 \\
50 \\
51 \\
53\end{array}$ & $\begin{array}{r}10.1 \\
9.4 \\
11.3 \\
10.8 \\
10.5\end{array}$ & $\begin{array}{l}13.9 \\
11.3 \\
14.7 \\
15.0 \\
14.7\end{array}$ & $\begin{array}{l}37.1 \\
25.3 \\
32.6 \\
37.8 \\
38.8\end{array}$ & $\begin{array}{r}-3.4 \\
3.0 \\
1.2 \\
-.4 \\
-3.1\end{array}$ \\
\hline $\begin{array}{r}6 \ldots \ldots \ldots \ldots \\
7 \ldots \ldots \ldots \\
8 \ldots \ldots \ldots \\
9 \ldots \ldots \ldots \ldots \\
10 \ldots \ldots \ldots \ldots\end{array}$ & $\begin{array}{r}194 \\
144 \\
96 \\
195 \\
32\end{array}$ & $\begin{array}{r}50 \\
34 \\
21 \\
46 \\
0\end{array}$ & $\begin{array}{r}9.7 \\
8.9 \\
10.0 \\
7.1 \\
5.0\end{array}$ & $\begin{array}{r}13.4 \\
11.8 \\
11.6 \\
10.1 \\
6.7\end{array}$ & $\begin{array}{r}36.1 \\
31.7 \\
22.3 \\
22.3 \\
9.6\end{array}$ & $\begin{array}{r}-2.9 \\
-1.0 \\
4.5 \\
-3.0 \\
4.0\end{array}$ \\
\hline $\begin{array}{l}11 \ldots \ldots \ldots \\
12 \ldots \ldots \ldots \\
13 \ldots \ldots \ldots \\
14 \ldots \ldots \ldots \ldots \\
15 \ldots \ldots \ldots \ldots\end{array}$ & $\begin{array}{l}194 \\
102 \\
147 \\
212 \\
204\end{array}$ & $\begin{array}{l}29 \\
14 \\
21 \\
31 \\
34\end{array}$ & $\begin{array}{l}6.0 \\
3.7 \\
3.7 \\
5.3 \\
3.7\end{array}$ & $\begin{array}{l}8.8 \\
5.7 \\
6.8 \\
9.0 \\
6.7\end{array}$ & $\begin{array}{l}22.8 \\
19.0 \\
23.8 \\
24.6 \\
21.8\end{array}$ & $\begin{array}{l}-.1 \\
-3 \cdot 0 \\
-4.3 \\
-1.9 \\
-5.7\end{array}$ \\
\hline $\begin{array}{l}16 \ldots \ldots \ldots \ldots \\
17 \ldots \ldots \ldots \\
18 \ldots \ldots \ldots \\
19 \ldots \ldots \ldots \ldots \\
20 \ldots \ldots \ldots \ldots\end{array}$ & $\begin{array}{r}124 \\
-- \\
-- \\
-- \\
243\end{array}$ & $\begin{array}{l}14 \\
-- \\
-- \\
-- \\
57\end{array}$ & $\begin{array}{l}4.1 \\
-- \\
-- \\
--1\end{array}$ & $\begin{array}{l}6.4 \\
-- \\
-- \\
-\overline{1} \\
12.6\end{array}$ & $\begin{array}{c}16.9 \\
-- \\
-- \\
34.6\end{array}$ & $\begin{array}{l}1.8 \\
-- \\
-- \\
-- \\
-2.5\end{array}$ \\
\hline $\begin{array}{l}21 \ldots \ldots \ldots \ldots \\
22 \ldots \ldots \ldots \ldots \\
23 \ldots \ldots \ldots \\
24 \ldots \ldots \ldots \ldots \\
25 \ldots \ldots \ldots \ldots\end{array}$ & $\begin{array}{l}247 \\
250 \\
192 \\
203 \\
247\end{array}$ & $\begin{array}{l}62 \\
66 \\
48 \\
54 \\
68\end{array}$ & $\begin{array}{r}7.8 \\
8.1 \\
9.3 \\
11.9 \\
11.7\end{array}$ & $\begin{array}{l}12.2 \\
12.6 \\
12.9 \\
15.1 \\
15.5\end{array}$ & $\begin{array}{l}33.8 \\
35.2 \\
34.6 \\
32.4 \\
36.6\end{array}$ & $\begin{array}{r}-4 \cdot 8 \\
-5 \cdot 5 \\
-4 \cdot 1 \\
4.4 \\
-2.3\end{array}$ \\
\hline $\begin{array}{l}26 \ldots \ldots \ldots \ldots \\
27 \ldots \ldots \ldots \\
28 \ldots \ldots \ldots \ldots \\
29 \ldots \ldots \ldots \ldots \\
30 \ldots \ldots \ldots \ldots\end{array}$ & $\begin{array}{r}247 \\
249 \\
206 \\
91 \\
240\end{array}$ & $\begin{array}{l}68 \\
69 \\
55 \\
18 \\
66\end{array}$ & $\begin{array}{l}11.7 \\
11.7 \\
14.2 \\
16.2 \\
13.6\end{array}$ & $\begin{array}{l}16.4 \\
16.4 \\
17.7 \\
19.0 \\
17.8\end{array}$ & $\begin{array}{l}39.1 \\
40.5 \\
40.5 \\
32.6 \\
36.6\end{array}$ & $\begin{array}{r}-.8 \\
-2.6 \\
-1.1 \\
9.0 \\
1.4\end{array}$ \\
\hline $31 \ldots \ldots \ldots$ & 140 & 29 & 13.8 & 16.5 & 34.4 & 1.8 \\
\hline Mean......... & $\overline{181}$ & $\overline{42}$ & 9.2 & $\overline{12.5}$ & $\overline{30.5}$ & -.8 \\
\hline
\end{tabular}

Apr11 1986

\begin{tabular}{|c|c|c|c|c|c|c|}
\hline $\begin{array}{l}1 \ldots \ldots \ldots \\
2 \ldots \ldots \ldots \\
3 \ldots \ldots \ldots \\
4 \ldots \ldots \ldots \\
5 \ldots \ldots \ldots\end{array}$ & $\begin{array}{l}216 \\
258 \\
267 \\
239 \\
196\end{array}$ & $\begin{array}{l}47 \\
49 \\
47 \\
42 \\
33\end{array}$ & $\begin{array}{r}15.0 \\
8.7 \\
10.3 \\
8.7 \\
10.0\end{array}$ & $\begin{array}{l}17.8 \\
10.9 \\
14.3 \\
12.7 \\
13.2\end{array}$ & $\begin{array}{l}31.0 \\
23.0 \\
33.0 \\
30.6 \\
27.9\end{array}$ & $\begin{array}{r}5.8 \\
-.9 \\
-1.3 \\
-3.5 \\
-1.6\end{array}$ \\
\hline $\begin{array}{c}6 \ldots \ldots \ldots \\
7 \ldots \ldots \ldots \\
8 \ldots \ldots \ldots \\
9 \ldots \ldots \ldots \ldots \\
10 \ldots \ldots \ldots \ldots \\
\ldots \ldots \ldots \ldots \ldots\end{array}$ & $\begin{array}{r}80 \\
221 \\
242 \\
252 \\
219\end{array}$ & $\begin{array}{r}8 \\
30 \\
35 \\
38 \\
33\end{array}$ & $\begin{array}{r}6.5 \\
8.1 \\
9.5 \\
10.1 \\
10.9\end{array}$ & $\begin{array}{r}8.8 \\
11.7 \\
14.0 \\
15.7 \\
15.3\end{array}$ & $\begin{array}{l}22.3 \\
24.7 \\
31.0 \\
37.1 \\
35.5\end{array}$ & $\begin{array}{r}.9 \\
-.2 \\
1.4 \\
-1.7 \\
.5\end{array}$ \\
\hline $\begin{array}{l}11 \ldots \ldots \ldots \\
12 \ldots \ldots \ldots \\
13 \ldots \ldots \ldots \\
14 \ldots \ldots \ldots \ldots \\
15 \ldots \ldots \ldots\end{array}$ & $\begin{array}{l}275 \\
281 \\
299 \\
193 \\
270\end{array}$ & $\begin{array}{l}56 \\
75 \\
90 \\
53 \\
82\end{array}$ & $\begin{array}{r}11.5 \\
11.5 \\
9.4 \\
9.9 \\
12.2\end{array}$ & $\begin{array}{l}16.1 \\
14.2 \\
12.3 \\
13.3 \\
15.1\end{array}$ & $\begin{array}{l}37.4 \\
29.7 \\
27.6 \\
30.3 \\
30.2\end{array}$ & $\begin{array}{r}-1.9 \\
-.2 \\
-.8 \\
-1.5 \\
-1.3\end{array}$ \\
\hline $\begin{array}{l}16 \ldots \ldots \ldots \ldots \\
17 \ldots \ldots \ldots \\
18 \ldots \ldots \ldots \\
19 \ldots \ldots \ldots \ldots \\
20 \ldots \ldots \ldots \ldots \ldots\end{array}$ & $\begin{array}{l}145 \\
246 \\
295 \\
290 \\
292\end{array}$ & $\begin{array}{l}42 \\
66 \\
82 \\
79 \\
78\end{array}$ & $\begin{array}{r}8.1 \\
8.5 \\
8.7 \\
10.1 \\
15.8\end{array}$ & $\begin{array}{l}10.7 \\
11.3 \\
11.9 \\
14.3 \\
--\end{array}$ & $\begin{array}{l}22.7 \\
28.2 \\
28.4 \\
36.4 \\
--\end{array}$ & $\begin{array}{l}-1.6 \\
-5.4 \\
-2.1 \\
-3.8 \\
-\end{array}$ \\
\hline
\end{tabular}


Table 3. Radiation and temperature field data for crater 3, June 1985 through June 1986 -Continued

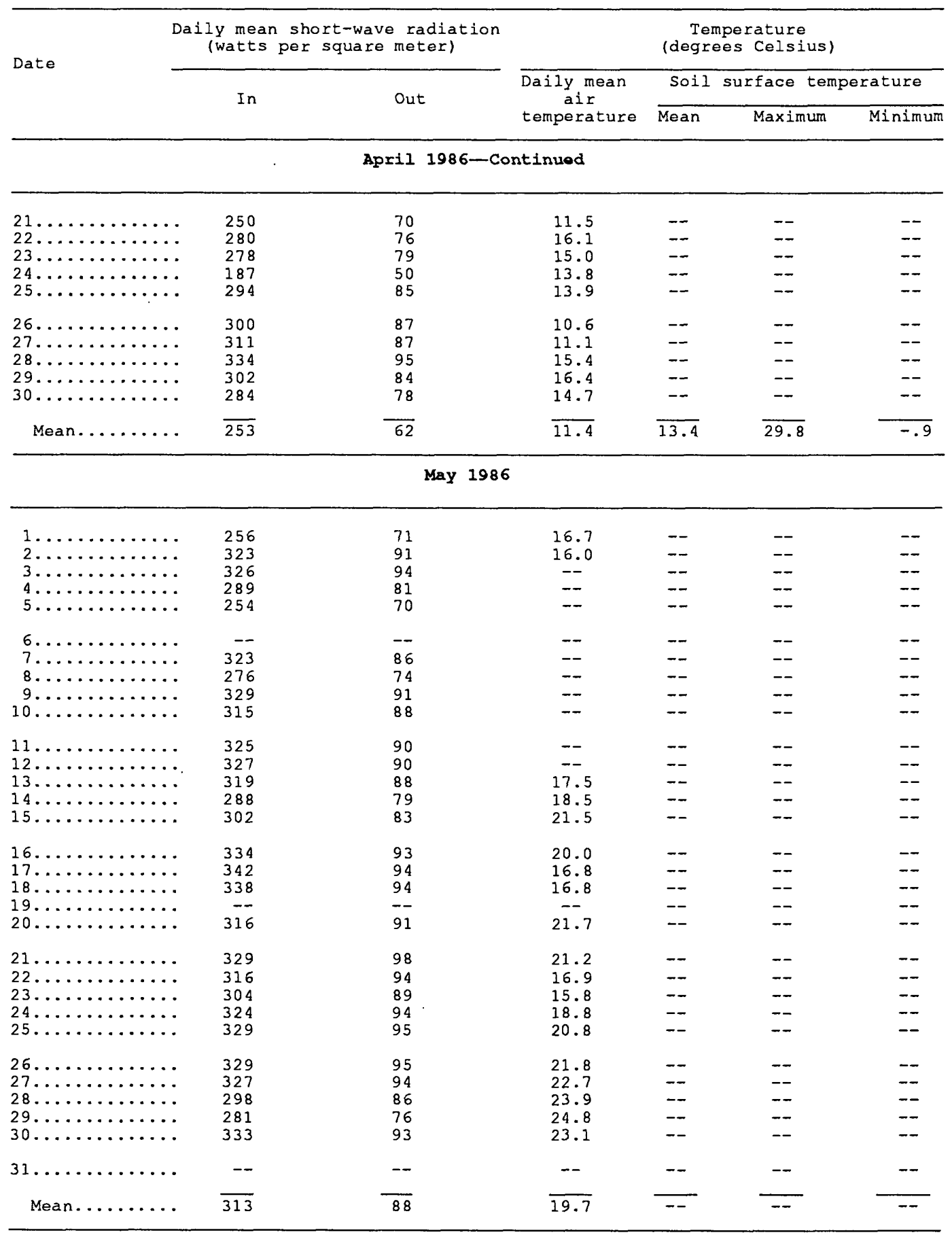


Table 3. Radiation and temperature field data for crater 3, June 1985 through June 1986 -Continued

\begin{tabular}{|c|c|c|c|c|c|c|}
\hline \multirow{3}{*}{ Date } & \multicolumn{2}{|c|}{$\begin{array}{l}\text { Daily mean short-wave radiation } \\
\text { (watts per square meter) }\end{array}$} & \multicolumn{4}{|c|}{$\begin{array}{l}\text { Temperature } \\
\text { (degrees Celsius) }\end{array}$} \\
\hline & \multirow{3}{*}{ In } & \multirow{2}{*}{ out } & \multirow{2}{*}{$\begin{array}{c}\text { Daily mean } \\
\text { air } \\
\text { temperature }\end{array}$} & \multicolumn{2}{|c|}{ Soil surface } & temperature \\
\hline & & & & Mean & Maximum & Minimum \\
\hline \multicolumn{6}{|c|}{ June 1986} & \\
\hline $\begin{array}{l}1 \ldots \ldots \\
2 \ldots \ldots \\
3 \ldots \ldots \ldots \\
4 \ldots \ldots \\
5 \ldots \ldots\end{array}$ & $\begin{array}{l}318 \\
281 \\
326 \\
288 \\
348\end{array}$ & $\begin{array}{l}86 \\
78 \\
87 \\
78 \\
98\end{array}$ & $\begin{array}{l}-- \\
-- \\
25.0 \\
24.8 \\
22.2\end{array}$ & $\begin{array}{l}-- \\
-- \\
-- \\
--\end{array}$ & $\begin{array}{l}-- \\
-- \\
-- \\
-- \\
--\end{array}$ & $\begin{array}{l}-- \\
-- \\
-- \\
-- \\
--\end{array}$ \\
\hline $\begin{array}{c}6 \ldots \ldots \ldots \\
7 \ldots \ldots \ldots \\
8 \ldots \ldots \ldots \\
9 \ldots \ldots \ldots \\
10 \ldots \ldots \ldots \ldots \\
\ldots \ldots \ldots \ldots \ldots\end{array}$ & $\begin{array}{l}343 \\
341 \\
324 \\
346 \\
341\end{array}$ & $\begin{array}{l}95 \\
96 \\
89 \\
96 \\
94\end{array}$ & $\begin{array}{l}19.8 \\
20.4 \\
23.5 \\
23.0 \\
20.7\end{array}$ & $\begin{array}{l}-- \\
-- \\
-- \\
-- \\
--\end{array}$ & $\begin{array}{l}-- \\
-- \\
-- \\
-- \\
--\end{array}$ & $\begin{array}{l}-- \\
-- \\
-- \\
-- \\
--\end{array}$ \\
\hline $\begin{array}{l}11 \ldots \ldots \ldots \ldots \\
12 \ldots \ldots \ldots \\
13 \ldots \ldots \ldots \ldots \\
14 \ldots \ldots \ldots \ldots \\
15 \ldots \ldots \ldots \ldots \ldots\end{array}$ & $\begin{array}{l}340 \\
339 \\
343 \\
320 \\
299\end{array}$ & $\begin{array}{l}94 \\
96 \\
96 \\
91 \\
85\end{array}$ & $\begin{array}{l}21.6 \\
22.0 \\
21.9 \\
22.5 \\
21.0\end{array}$ & $\begin{array}{l}-- \\
-- \\
-- \\
-- \\
--\end{array}$ & $\begin{array}{l}-- \\
-- \\
-- \\
-- \\
--\end{array}$ & $\begin{array}{l}-- \\
-- \\
-- \\
-- \\
--\end{array}$ \\
\hline $\begin{array}{l}16 \ldots \ldots \ldots \\
17 \ldots \ldots \ldots \\
18 \ldots \ldots \ldots \\
19 \ldots \ldots \ldots \\
20 \ldots \ldots \ldots \ldots\end{array}$ & $\begin{array}{l}3 \overline{5} \\
350 \\
350 \\
352\end{array}$ & $\begin{array}{l}103 \\
101 \\
101 \\
103\end{array}$ & $\begin{array}{l}-- \\
22.0 \\
22.1 \\
21.1 \\
20.5\end{array}$ & $\begin{array}{l}-- \\
-- \\
-- \\
-- \\
--\end{array}$ & $\begin{array}{l}-- \\
-- \\
-- \\
-- \\
--\end{array}$ & $\begin{array}{l}-- \\
-- \\
-- \\
-- \\
--\end{array}$ \\
\hline $\begin{array}{l}21 \ldots \ldots \\
22 \ldots \ldots \ldots \\
23 \ldots \ldots \ldots \\
24 \ldots \ldots \ldots \\
25 \ldots \ldots \ldots\end{array}$ & $\begin{array}{l}345 \\
346 \\
308 \\
343 \\
341\end{array}$ & $\begin{array}{r}99 \\
99 \\
88 \\
100 \\
99\end{array}$ & $\begin{array}{l}21.3 \\
22.2 \\
23.2 \\
24.7 \\
26.8\end{array}$ & $\begin{array}{l}-- \\
-- \\
-- \\
-- \\
--\end{array}$ & $\begin{array}{l}-- \\
-- \\
-- \\
-- \\
--\end{array}$ & $\begin{array}{l}-- \\
-- \\
-- \\
-- \\
--\end{array}$ \\
\hline $\begin{array}{l}26 \ldots \ldots \ldots \\
27 \ldots \ldots \ldots \\
28 \ldots \ldots \ldots \\
29 \ldots \ldots \ldots \ldots \\
30 \ldots \ldots \ldots \ldots\end{array}$ & $\begin{array}{l}331 \\
254 \\
299 \\
340 \\
--\end{array}$ & $\begin{array}{l}97 \\
73 \\
86 \\
99 \\
--\end{array}$ & $\begin{array}{l}25.9 \\
25.6 \\
26.1 \\
25.9 \\
--\end{array}$ & $\begin{array}{l}-- \\
-- \\
-- \\
-- \\
--\end{array}$ & $\begin{array}{l}-- \\
-- \\
-- \\
-- \\
--\end{array}$ & $\begin{array}{l}-- \\
-- \\
-- \\
-- \\
--\end{array}$ \\
\hline Mean......... & $\overline{329}$ & 93 & $\overline{22.9}$ & -- & -- & -- \\
\hline
\end{tabular}


Table 4. Meteorological data for undisturbed-land-surface site, May 1985 through June 1986

[Footnotes on this page apply to entire table. --, no data or not calculated]

\begin{tabular}{|c|c|c|c|c|c|c|}
\hline \multirow{2}{*}{ Date } & \multirow{2}{*}{$\begin{array}{c}\text { Daily mean } \\
\text { air } \\
\text { temperature } \\
\text { (degrees } \\
\text { Celsius) }\end{array}$} & \multirow{2}{*}{$\begin{array}{c}\text { Daily } \\
\text { mean } \\
\text { relative } \\
\text { humidity } \\
\text { (percent) }\end{array}$} & \multicolumn{2}{|c|}{$\begin{array}{l}\text { Wind velocity } \\
\text { (meters per second) }\end{array}$} & \multicolumn{2}{|c|}{$\begin{array}{l}\text { Wind direction } \\
\text { (degrees) }\end{array}$} \\
\hline & & & $\begin{array}{l}\text { Daily } \\
\text { maximum }\end{array}$ & $\begin{array}{l}\text { Daily } \\
\text { mean }\end{array}$ & $\begin{array}{l}\text { Maximum } \\
\text { mean }\end{array}$ & $\begin{array}{l}\text { Daily } \\
\text { mean }\end{array}$ \\
\hline \multicolumn{7}{|c|}{ May 1985} \\
\hline $\begin{array}{l}1 \ldots \ldots \cdots \\
2 \ldots \ldots \cdots \\
3 \ldots \ldots \cdots \\
5 \ldots \ldots \cdots\end{array}$ & $\begin{array}{l}-- \\
-- \\
--\end{array}$ & $\begin{array}{l}26.7 \\
27.2 \\
19.7 \\
20.2 \\
24.3\end{array}$ & $\begin{array}{l}1.73 \\
2.90 \\
3.86 \\
2.66 \\
2.16\end{array}$ & $\begin{array}{r}0.47 \\
.16 \\
.14 \\
.29 \\
.32\end{array}$ & $\begin{array}{l}292 \\
285 \\
291 \\
257 \\
295\end{array}$ & $\begin{array}{l}200 \\
246 \\
256 \\
210 \\
232\end{array}$ \\
\hline $\begin{array}{r}6 \ldots \ldots \ldots \\
7 \ldots \ldots \\
8 \ldots \ldots \cdots \\
9 \ldots \ldots \\
10 \ldots \ldots\end{array}$ & $\begin{array}{l}-- \\
-- \\
-- \\
--\end{array}$ & $\begin{array}{l}17.6 \\
21.2 \\
16.3 \\
40.5 \\
43.8\end{array}$ & $\begin{array}{l}4.77 \\
3.68 \\
4.39 \\
4.92 \\
4.87\end{array}$ & $\begin{array}{l}.11 \\
.15 \\
.10 \\
.07 \\
.34\end{array}$ & $\begin{array}{l}300 \\
304 \\
310 \\
309 \\
263\end{array}$ & $\begin{array}{l}276 \\
252 \\
311 \\
310 \\
232\end{array}$ \\
\hline $\begin{array}{l}11 \ldots \ldots \ldots \\
12 \ldots \ldots \ldots\end{array}$ & -- & $\begin{array}{l}37.2 \\
28.1\end{array}$ & $\begin{array}{l}2.09 \\
2.95\end{array}$ & $\begin{array}{l}.24 \\
.49\end{array}$ & $\begin{array}{l}292 \\
218\end{array}$ & $\begin{array}{l}276 \\
139\end{array}$ \\
\hline $13 \ldots \ldots \ldots$ & - & -- & -- & -- & -- & -- \\
\hline $14 \ldots \ldots \ldots$ & -- & -- & - & -- & -- & -- \\
\hline $15 \ldots \ldots \ldots$ & - & - & - & -- & - & - \\
\hline $16 \ldots \ldots \ldots$ & -- & -- & - & -- & -- & -- \\
\hline $\begin{array}{l}17 \ldots \ldots \\
18 \ldots \ldots\end{array}$ & -- & $\begin{array}{l}-- \\
--\end{array}$ & $=$ & $\overline{--}$ & $=$ & $=-$ \\
\hline $19 \ldots \ldots \ldots$ & -- & -- & -- & -- & -- & - \\
\hline $20 \ldots \ldots \ldots$ & - & - & - & -- & -- & - \\
\hline $21 \ldots \ldots \ldots$ & -- & 28.2 & 3.17 & .20 & 196 & 158 \\
\hline $22 \ldots \ldots \ldots$ & -- & 30.0 & 1.59 & .45 & 282 & 220 \\
\hline $23 \ldots \ldots \ldots$ & - & 24.7 & 1.97 & .29 & 252 & 258 \\
\hline $24 \ldots \ldots \ldots$ & - & 22.4 & 2.86 & .35 & 290 & 256 \\
\hline $25 \ldots \ldots \ldots$ & $-\infty$ & 20.9 & 2.97 & .20 & 296 & 253 \\
\hline $26 \ldots \ldots \ldots$ & - & 18.9 & 3.41 & .35 & 291 & 247 \\
\hline $27 \ldots \ldots \ldots$ & - & 16.3 & 3.31 & .18 & 299 & 263 \\
\hline $28 \ldots \ldots \ldots$ & -- & 15.1 & 4.11 & .14 & 309 & 297 \\
\hline $29 \ldots \ldots \ldots$ & -- & 13.8 & 4.30 & .16 & 301 & 265 \\
\hline $30 \ldots \ldots \ldots$ & - & 11.7 & 3.28 & .13 & 250 & 215 \\
\hline $31 \ldots \ldots \ldots$ & -- & 21.6 & 3.38 & .24 & 267 & 254 \\
\hline Mean...... & - & 23.7 & 3.27 & .24 & 280 & 245 \\
\hline
\end{tabular}

June 1985

\begin{tabular}{|c|c|c|c|c|c|c|}
\hline $\begin{array}{l}1 \ldots \ldots \\
2 \ldots \ldots \\
3 \ldots \ldots \\
4 \ldots \ldots \\
5 \ldots \ldots\end{array}$ & $\begin{array}{l}-- \\
-- \\
-- \\
-- \\
--\end{array}$ & $\begin{array}{l}28.3 \\
24.5 \\
52.2 \\
46.0 \\
34.4\end{array}$ & $\begin{array}{l}2.43 \\
2.89 \\
2.20 \\
1.49 \\
2.20\end{array}$ & $\begin{array}{r}0.23 \\
.21 \\
.44 \\
.36 \\
.23\end{array}$ & $\begin{array}{l}286 \\
293 \\
233 \\
280 \\
286\end{array}$ & $\begin{array}{l}259 \\
232 \\
205 \\
252 \\
259\end{array}$ \\
\hline $\begin{array}{r}6 \ldots \ldots \ldots \\
7 \ldots \ldots \ldots \\
8 \ldots \ldots \ldots \\
9 \ldots \ldots \ldots \\
10 \ldots \ldots \ldots\end{array}$ & $\begin{array}{l}-- \\
-- \\
-- \\
-- \\
--\end{array}$ & $\begin{array}{l}30.5 \\
25.8 \\
20.9 \\
20.0 \\
16.2\end{array}$ & $\begin{array}{l}2.04 \\
2.38 \\
2.27 \\
2.04 \\
2.21\end{array}$ & $\begin{array}{l}.38 \\
.36 \\
.28 \\
.39 \\
.62\end{array}$ & $\begin{array}{l}300 \\
239 \\
266 \\
256 \\
193\end{array}$ & $\begin{array}{r}207 \\
-- \\
-- \\
223 \\
101\end{array}$ \\
\hline $\begin{array}{l}11 \ldots \ldots \ldots \\
12 \ldots \ldots \\
13 \ldots \ldots \ldots \\
14 \ldots \ldots \ldots \\
15 \ldots \ldots \ldots\end{array}$ & $\begin{array}{l}-- \\
-- \\
-- \\
-- \\
--\end{array}$ & $\begin{array}{l}12.4 \\
16.2 \\
17.4 \\
16.4 \\
16.1\end{array}$ & $\begin{array}{l}2.45 \\
1.87 \\
1.99 \\
1.69 \\
1.76\end{array}$ & $\begin{array}{l}.23 \\
.30 \\
.27 \\
.28 \\
.24\end{array}$ & $\begin{array}{l}230 \\
282 \\
281 \\
284 \\
279\end{array}$ & $\begin{array}{l}208 \\
256 \\
232 \\
234 \\
238\end{array}$ \\
\hline
\end{tabular}

${ }^{l}$ Daily maximum values are means of hourly maximum values for a given day; dally mean values were derived from all velocity readings for a given day.

${ }^{2}$ Maximum mean values are daily means of hourly azimuth readings that correspond to hourly maximum wind velocities; daily mean values were derived from all azimuth readings for a given day. 
Table 4. Meteorological data for undisturbed-land-surface site, May 1985 through June 1986 -Continued

\begin{tabular}{|c|c|c|c|c|c|c|}
\hline \multirow{2}{*}{ Date } & \multirow{2}{*}{$\begin{array}{c}\text { Daily mean } \\
\text { air } \\
\text { temperature } \\
\text { (degrees } \\
\text { Celsius) }\end{array}$} & \multirow{2}{*}{$\begin{array}{c}\text { Daily } \\
\text { mean } \\
\text { relative } \\
\text { humidity } \\
\text { (percent) }\end{array}$} & \multicolumn{2}{|c|}{$\begin{array}{l}\text { Wind velocity } \\
\text { (meters per second) }\end{array}$} & \multicolumn{2}{|c|}{$\begin{array}{l}\text { Wind direction } \\
\text { (degrees) }\end{array}$} \\
\hline & & & $\begin{array}{l}\text { Daily } \\
\text { maximum }\end{array}$ & $\begin{array}{l}\text { Daily } \\
\text { mean }\end{array}$ & $\begin{array}{l}\text { Maximum } \\
\text { mean }\end{array}$ & $\begin{array}{l}\text { Daily } \\
\text { mean }\end{array}$ \\
\hline \multicolumn{7}{|c|}{ June $1985-$ Continued } \\
\hline $\begin{array}{l}16 \ldots \ldots \ldots \\
17 \ldots \ldots \ldots \\
18 \ldots \ldots \ldots \\
19 \ldots \ldots \ldots \\
20 \ldots \ldots \ldots \ldots\end{array}$ & $\begin{array}{l}-- \\
-- \\
-- \\
-- \\
--\end{array}$ & $\begin{array}{l}14.8 \\
13.3 \\
14.3 \\
15.5 \\
14.6\end{array}$ & $\begin{array}{l}1.70 \\
1.46 \\
1.64 \\
2.38 \\
2.45\end{array}$ & $\begin{array}{r}0.32 \\
.36 \\
.40 \\
.29 \\
.35\end{array}$ & $\begin{array}{l}272 \\
293 \\
286 \\
283 \\
270\end{array}$ & $\begin{array}{l}257 \\
227 \\
216 \\
242 \\
224\end{array}$ \\
\hline $\begin{array}{l}21 \ldots \ldots \ldots \\
22 \ldots \ldots \ldots \\
23 \ldots \ldots \ldots \\
24 \ldots \ldots \\
25 \ldots \ldots \ldots\end{array}$ & $\begin{array}{l}-- \\
-- \\
-- \\
-- \\
--\end{array}$ & $\begin{array}{l}10.5 \\
11.8 \\
15.0 \\
21.5 \\
16.4\end{array}$ & $\begin{array}{l}1.74 \\
2.87 \\
2.60 \\
2.73 \\
3.82\end{array}$ & $\begin{array}{l}.26 \\
.30 \\
.14 \\
.18 \\
.43\end{array}$ & $\begin{array}{l}294 \\
295 \\
252 \\
278 \\
169\end{array}$ & $\begin{array}{l}233 \\
232 \\
261 \\
256 \\
158\end{array}$ \\
\hline $\begin{array}{l}26 \ldots \ldots \ldots \\
27 \ldots \ldots \ldots \\
28 \ldots \ldots \ldots \\
29 \ldots \ldots \ldots \\
30 \ldots \ldots \ldots\end{array}$ & $\begin{array}{l}-- \\
-- \\
-- \\
-- \\
--\end{array}$ & $\begin{array}{r}10.5 \\
11.6 \\
11.1 \\
11.6 \\
9.8\end{array}$ & $\begin{array}{l}2.07 \\
1.78 \\
2.40 \\
2.74 \\
2.35\end{array}$ & $\begin{array}{l}.34 \\
.48 \\
.35 \\
.31 \\
.37\end{array}$ & $\begin{array}{l}214 \\
245 \\
276 \\
289 \\
278\end{array}$ & $\begin{array}{l}199 \\
183 \\
238 \\
224 \\
241\end{array}$ \\
\hline Mean..... & -- & $\overline{19.3}$ & $\overline{2.22}$ & .32 & $\overline{266}$ & $\overline{225}$ \\
\hline \multicolumn{7}{|c|}{ July 1985} \\
\hline $\begin{array}{l}1 \ldots \ldots \\
2 \ldots \ldots \\
3 \ldots \ldots \\
4 \ldots \ldots \\
5 \ldots \ldots\end{array}$ & $\begin{array}{l}-- \\
-- \\
-- \\
-- \\
--\end{array}$ & $\begin{array}{l}11.9 \\
11.1 \\
10.3 \\
11.2 \\
12.4\end{array}$ & $\begin{array}{l}1.58 \\
1.26 \\
1.75 \\
2.03 \\
1.68\end{array}$ & $\begin{array}{r}0.39 \\
.41 \\
.37 \\
.26 \\
.28\end{array}$ & $\begin{array}{l}273 \\
279 \\
292 \\
268 \\
282\end{array}$ & $\begin{array}{l}228 \\
232 \\
220 \\
249 \\
250\end{array}$ \\
\hline $\begin{array}{c}6 \ldots \ldots \ldots \\
7 \ldots \ldots \\
8 \ldots \ldots \ldots \\
9 \ldots \ldots \\
10 \ldots \ldots\end{array}$ & $\begin{array}{l}-- \\
-- \\
-- \\
-- \\
--\end{array}$ & $\begin{array}{l}12.4 \\
24.6 \\
28.4 \\
19.0 \\
23.1\end{array}$ & $\begin{array}{l}2.28 \\
2.04 \\
1.59 \\
2.59 \\
2.30\end{array}$ & $\begin{array}{l}.23 \\
.44 \\
.30 \\
.31 \\
.18\end{array}$ & $\begin{array}{l}292 \\
251 \\
264 \\
290 \\
290\end{array}$ & $\begin{array}{l}267 \\
211 \\
257 \\
272 \\
284\end{array}$ \\
\hline $\begin{array}{l}11 \ldots \ldots \ldots \\
12 \ldots \ldots \ldots \\
13 \ldots \ldots \ldots \\
14 \ldots \ldots \ldots \\
15 \ldots \ldots \ldots\end{array}$ & $\begin{array}{l}-- \\
-- \\
-- \\
-- \\
--\end{array}$ & $\begin{array}{l}21.7 \\
23.0 \\
18.5 \\
16.8 \\
18.1\end{array}$ & $\begin{array}{l}2.22 \\
2.29 \\
1.84 \\
2.35 \\
2.52\end{array}$ & $\begin{array}{l}.28 \\
.31 \\
.45 \\
.27 \\
.23\end{array}$ & $\begin{array}{l}276 \\
260 \\
288 \\
294 \\
272\end{array}$ & $\begin{array}{l}245 \\
231 \\
240 \\
249 \\
236\end{array}$ \\
\hline $\begin{array}{l}16 \ldots \ldots \ldots \\
17 \ldots \ldots \ldots \\
18 \ldots \ldots \ldots \\
19 \ldots \ldots \\
20 \ldots \ldots \ldots\end{array}$ & $\begin{array}{l}-- \\
-- \\
-- \\
-- \\
--\end{array}$ & $\begin{array}{l}27.9 \\
31.6 \\
-- \\
67.9 \\
75.8\end{array}$ & $\begin{array}{l}2.25 \\
2.64 \\
-- \\
1.68 \\
1.88\end{array}$ & $\begin{array}{r}.15 \\
.21 \\
-- \\
.35 \\
.23\end{array}$ & $\begin{array}{r}293 \\
296 \\
-- \\
279 \\
253\end{array}$ & $\begin{array}{r}238 \\
238 \\
-- \\
192 \\
201\end{array}$ \\
\hline $\begin{array}{l}21 \ldots \ldots \ldots \\
22 \ldots \ldots \\
23 \ldots \ldots \ldots \\
24 \ldots \ldots \\
25 \ldots \ldots \ldots\end{array}$ & $\begin{array}{l}-- \\
-- \\
-- \\
-- \\
--\end{array}$ & $\begin{array}{l}69.3 \\
49.5 \\
32.4 \\
26.6 \\
29.7\end{array}$ & $\begin{array}{l}1.68 \\
1.80 \\
2.12 \\
1.70 \\
1.93\end{array}$ & $\begin{array}{l}.21 \\
.30 \\
.34 \\
.27 \\
.32\end{array}$ & $\begin{array}{l}272 \\
270 \\
268 \\
263 \\
258\end{array}$ & $\begin{array}{l}269 \\
250 \\
221 \\
239 \\
234\end{array}$ \\
\hline $\begin{array}{l}26 \ldots \ldots \ldots \\
27 \ldots \ldots \ldots \\
28 \ldots \ldots \ldots \\
29 \ldots \ldots \\
30 \ldots \ldots\end{array}$ & $\begin{array}{l}-- \\
-- \\
-- \\
-- \\
--\end{array}$ & $\begin{array}{l}27.9 \\
25.3 \\
24.1 \\
21.4 \\
16.9\end{array}$ & $\begin{array}{l}1.47 \\
1.97 \\
2.54 \\
3.58 \\
2.51\end{array}$ & $\begin{array}{l}.40 \\
.22 \\
.20 \\
.22 \\
.19\end{array}$ & $\begin{array}{l}245 \\
299 \\
294 \\
278 \\
301\end{array}$ & $\begin{array}{l}227 \\
256 \\
250 \\
271 \\
275\end{array}$ \\
\hline $31 \ldots \ldots \ldots$ & -- & 19.6 & 3.68 & .20 & 277 & 286 \\
\hline Mean...... & -- & $\overline{26.9}$ & $\overline{2.13}$ & .28 & $\overline{277}$ & $\overline{244}$ \\
\hline
\end{tabular}


Table 4. Meteorological data for undisturbed-land-surface site, May 1985 through June 1986 -Continued

\begin{tabular}{|c|c|c|c|c|c|c|}
\hline \multirow{2}{*}{ Date } & \multirow{2}{*}{$\begin{array}{c}\text { Daily mean } \\
\text { air } \\
\text { temperature } \\
\text { (degrees } \\
\text { Celsius) }\end{array}$} & \multirow{2}{*}{$\begin{array}{l}\text { Daily } \\
\text { mean } \\
\text { relative } \\
\text { humidity } \\
\text { (percent) }\end{array}$} & \multicolumn{2}{|c|}{$\begin{array}{l}\text { Wind velocity } \\
\text { (meters per second) }\end{array}$} & \multicolumn{2}{|c|}{$\begin{array}{l}\text { Wind direction } \\
\text { (degrees) }\end{array}$} \\
\hline & & & $\begin{array}{l}\text { Daily } \\
\text { maximum }\end{array}$ & $\begin{array}{l}\text { Daily } \\
\text { mean }\end{array}$ & $\begin{array}{l}\text { Maximum } \\
\text { mean }\end{array}$ & $\begin{array}{l}\text { Daily } \\
\text { mean }\end{array}$ \\
\hline \multicolumn{7}{|c|}{ Auguat 1985} \\
\hline $\begin{array}{l}1 \ldots \ldots \\
2 \ldots \ldots \\
3 \ldots \ldots \\
4 \ldots \ldots \\
5 \ldots \ldots\end{array}$ & $\begin{array}{l}-- \\
-- \\
-- \\
-- \\
--\end{array}$ & $\begin{array}{l}15.8 \\
17.5 \\
17.2 \\
16.7 \\
15.4\end{array}$ & $\begin{array}{l}3.84 \\
1.95 \\
1.50 \\
2.07 \\
1.75\end{array}$ & $\begin{array}{r}0.15 \\
.41 \\
.47 \\
.31 \\
.51\end{array}$ & $\begin{array}{l}297 \\
251 \\
298 \\
278 \\
264\end{array}$ & $\begin{array}{l}285 \\
251 \\
211 \\
205 \\
209\end{array}$ \\
\hline $\begin{array}{c}6 \ldots \ldots \ldots \\
7 \ldots \ldots \ldots \\
8 \ldots \ldots \\
9 \ldots \ldots \ldots \\
10 \ldots \ldots\end{array}$ & $\begin{array}{l}-- \\
-- \\
-- \\
-- \\
--\end{array}$ & $\begin{array}{l}15.0 \\
13.0 \\
12.8 \\
13.5 \\
15.0\end{array}$ & $\begin{array}{l}2.02 \\
2.82 \\
3.07 \\
2.51 \\
3.29\end{array}$ & $\begin{array}{l}.28 \\
.34 \\
.27 \\
.33 \\
.27\end{array}$ & $\begin{array}{l}284 \\
284 \\
269 \\
202 \\
286\end{array}$ & $\begin{array}{l}246 \\
231 \\
224 \\
181 \\
244\end{array}$ \\
\hline $\begin{array}{l}11 \ldots \ldots \ldots \\
12 \ldots \ldots \\
13 \ldots \ldots \\
14 \ldots \ldots \ldots \\
15 \ldots \ldots\end{array}$ & $\begin{array}{l}-- \\
-- \\
-- \\
-- \\
24.2\end{array}$ & $\begin{array}{l}16.2 \\
13.5 \\
15.7 \\
14.3 \\
14.8\end{array}$ & $\begin{array}{l}2.30 \\
2.54 \\
1.83 \\
1.34 \\
1.69\end{array}$ & $\begin{array}{l}.44 \\
.50 \\
.23 \\
.38 \\
.38\end{array}$ & $\begin{array}{l}230 \\
219 \\
302 \\
270 \\
270\end{array}$ & $\begin{array}{l}189 \\
185 \\
239 \\
267 \\
225\end{array}$ \\
\hline $\begin{array}{l}16 \ldots \ldots \ldots \\
17 \ldots \ldots \\
18 \ldots \ldots \\
19 \ldots \ldots \\
20 \ldots \ldots\end{array}$ & $\begin{array}{l}24.8 \\
24.5 \\
22.6 \\
22.1 \\
24.4\end{array}$ & $\begin{array}{l}16.3 \\
22.5 \\
18.4 \\
13.9 \\
13.3\end{array}$ & $\begin{array}{l}2.09 \\
2.81 \\
3.14 \\
2.47 \\
3.29\end{array}$ & $\begin{array}{l}.34 \\
.34 \\
.34 \\
.25 \\
.21\end{array}$ & $\begin{array}{l}279 \\
239 \\
290 \\
266 \\
267\end{array}$ & $\begin{array}{l}229 \\
225 \\
216 \\
268 \\
249\end{array}$ \\
\hline $\begin{array}{l}21 \ldots \ldots \ldots \\
22 \ldots \ldots \ldots \\
23 \ldots \ldots \ldots \\
24 \ldots \ldots \ldots \\
25 \ldots \ldots\end{array}$ & $\begin{array}{c}23.3 \\
-- \\
-- \\
-- \\
--\end{array}$ & $\begin{array}{c}14.5 \\
-- \\
-- \\
-- \\
--\end{array}$ & $\begin{array}{l}2.81 \\
-- \\
-- \\
-- \\
--\end{array}$ & $\begin{array}{l}.29 \\
-- \\
-- \\
--\end{array}$ & $\begin{array}{r}287 \\
-- \\
-- \\
-- \\
--\end{array}$ & $\begin{array}{r}248 \\
-- \\
-- \\
-- \\
--\end{array}$ \\
\hline $\begin{array}{l}26 \ldots \ldots \ldots \\
27 \ldots \ldots \ldots \\
28 \ldots \ldots \ldots \\
29 \ldots \ldots \ldots \\
30 \ldots \ldots \ldots\end{array}$ & $\begin{array}{l}-- \\
26.4 \\
24.5 \\
24.0 \\
25.3\end{array}$ & $\begin{array}{l}-- \\
24.5 \\
16.9 \\
15.2 \\
17.5\end{array}$ & $\begin{array}{l}-- \\
2.21 \\
2.22 \\
1.68 \\
2.21\end{array}$ & $\begin{array}{l}.42 \\
.40 \\
.46 \\
.35\end{array}$ & $\begin{array}{l}-7 \\
273 \\
253 \\
258 \\
287\end{array}$ & $\begin{array}{l}-\overline{2} \\
212 \\
231 \\
204 \\
227\end{array}$ \\
\hline $31 \ldots \ldots \ldots$ & 26.2 & 20.7 & 2.26 & .34 & 266 & 231 \\
\hline Mean..... & $\overline{24.3}$ & $\overline{16.2}$ & $\overline{2.37}$ & .35 & $\overline{268}$ & $\overline{228}$ \\
\hline \multicolumn{7}{|c|}{ September 1985} \\
\hline $\begin{array}{l}1 \ldots \ldots \ldots \\
2 \ldots \ldots \\
3 \ldots \ldots \\
4 \ldots \ldots \\
5 \ldots \ldots\end{array}$ & $\begin{array}{l}25.6 \\
25.9 \\
21.7 \\
16.7 \\
16.8\end{array}$ & $\begin{array}{l}21.1 \\
22.0 \\
29.1 \\
37.1 \\
41.1\end{array}$ & $\begin{array}{l}3.45 \\
3.65 \\
2.91 \\
1.82 \\
1.63\end{array}$ & $\begin{array}{r}0.23 \\
.20 \\
.26 \\
.32 \\
.24\end{array}$ & $\begin{array}{l}266 \\
307 \\
286 \\
296 \\
286\end{array}$ & $\begin{array}{l}258 \\
276 \\
275 \\
277 \\
275\end{array}$ \\
\hline $\begin{array}{r}6 \ldots \ldots \ldots \\
7 \ldots \ldots \ldots \\
8 \ldots \ldots \ldots \\
9 \ldots \ldots \ldots \\
10 \ldots \ldots \ldots\end{array}$ & $\begin{array}{l}14.7 \\
16.7 \\
19.0 \\
19.1 \\
15.5\end{array}$ & $\begin{array}{l}49.0 \\
44.7 \\
38.1 \\
32.4 \\
35.2\end{array}$ & $\begin{array}{l}1.96 \\
2.35 \\
3.17 \\
4.87 \\
2.84\end{array}$ & $\begin{array}{l}.36 \\
.26 \\
.23 \\
.10 \\
.26\end{array}$ & $\begin{array}{l}262 \\
268 \\
302 \\
307 \\
279\end{array}$ & $\begin{array}{l}206 \\
232 \\
248 \\
307 \\
237\end{array}$ \\
\hline $\begin{array}{l}11 \ldots \ldots \ldots \\
12 \ldots \ldots \ldots \\
13 \ldots \ldots \ldots \\
14 \ldots \ldots \ldots \\
15 \ldots \ldots \ldots\end{array}$ & $\begin{array}{l}12.6 \\
13.3 \\
16.5 \\
18.4 \\
18.8\end{array}$ & $\begin{array}{l}38.8 \\
29.3 \\
20.8 \\
15.7 \\
20.2\end{array}$ & $\begin{array}{l}2.99 \\
1.30 \\
1.80 \\
2.50 \\
2.21\end{array}$ & $\begin{array}{l}.36 \\
.38 \\
.35 \\
.46 \\
.50\end{array}$ & $\begin{array}{l}274 \\
258 \\
283 \\
292 \\
296\end{array}$ & $\begin{array}{l}273 \\
203 \\
209 \\
208 \\
202\end{array}$ \\
\hline
\end{tabular}


Table 4. Meteorological data for undisturbed-land-surface site, May 1985 through June 1986 -Continued

\begin{tabular}{|c|c|c|c|c|c|c|}
\hline \multirow{2}{*}{ Date } & \multirow{2}{*}{$\begin{array}{c}\text { Daily mean } \\
\text { air } \\
\text { temperature } \\
\text { (degrees } \\
\text { Celsius) }\end{array}$} & \multirow{2}{*}{$\begin{array}{l}\text { Daily } \\
\text { mean } \\
\text { relative } \\
\text { humidity } \\
\text { (percent) }\end{array}$} & \multicolumn{2}{|c|}{$\begin{array}{l}\text { Wind velocity } \\
\text { (meters per second) }\end{array}$} & \multicolumn{2}{|c|}{$\begin{array}{l}\text { Wind direction } \\
\text { (degrees) }\end{array}$} \\
\hline & & & $\begin{array}{l}\text { Daily } \\
\text { maximum }\end{array}$ & $\begin{array}{l}\text { Daily } \\
\text { mean }\end{array}$ & $\begin{array}{l}\text { Maximum } \\
\text { mean }\end{array}$ & $\begin{array}{l}\text { Daily } \\
\text { mean }\end{array}$ \\
\hline \multicolumn{7}{|c|}{ September 1985-Continued } \\
\hline $\begin{array}{l}16 \ldots \ldots \ldots \\
17 \ldots \ldots \ldots \\
18 \ldots \ldots \ldots \\
19 \ldots \ldots \\
20 \ldots \ldots \ldots\end{array}$ & $\begin{array}{l}17.8 \\
18.0 \\
15.8 \\
14.7 \\
14.8\end{array}$ & $\begin{array}{l}23.4 \\
26.8 \\
56.5 \\
67.3 \\
49.9\end{array}$ & $\begin{array}{l}2.40 \\
4.06 \\
2.07 \\
1.77 \\
1.65\end{array}$ & $\begin{array}{r}0.31 \\
.19 \\
.30 \\
.41 \\
.35\end{array}$ & $\begin{array}{r}267 \\
279 \\
287 \\
222 \\
282\end{array}$ & $\begin{array}{l}264 \\
275 \\
256 \\
144 \\
225\end{array}$ \\
\hline $\begin{array}{l}21 \ldots \ldots \ldots \\
22 \ldots \ldots \ldots \\
23 \ldots \ldots \ldots \\
24 \ldots \ldots \ldots \\
25 \ldots \ldots \ldots\end{array}$ & $\begin{array}{l}15.5 \\
17.0 \\
17.1 \\
19.2 \\
19.7\end{array}$ & $\begin{array}{l}38.5 \\
31.8 \\
27.5 \\
30.0 \\
24.6\end{array}$ & $\begin{array}{l}2.07 \\
2.49 \\
1.30 \\
1.82 \\
1.71\end{array}$ & $\begin{array}{l}.33 \\
.41 \\
.26 \\
.43 \\
.45\end{array}$ & $\begin{array}{l}281 \\
203 \\
273 \\
285 \\
236\end{array}$ & $\begin{array}{l}217 \\
168 \\
225 \\
250 \\
166\end{array}$ \\
\hline $\begin{array}{l}26 \ldots \ldots \ldots \\
27 \ldots \ldots \ldots \\
28 \ldots \ldots \ldots \\
29 \ldots \ldots \ldots \\
30 \ldots \ldots\end{array}$ & $\begin{array}{l}18.4 \\
18.6 \\
18.6 \\
14.9 \\
16.1\end{array}$ & $\begin{array}{l}25.2 \\
45.4 \\
45.9 \\
17.2 \\
30.8\end{array}$ & $\begin{array}{l}1.40 \\
2.06 \\
1.80 \\
1.55 \\
1.70\end{array}$ & $\begin{array}{l}.32 \\
.40 \\
.53 \\
.33 \\
.29\end{array}$ & $\begin{array}{l}271 \\
271 \\
192 \\
285 \\
278\end{array}$ & $\begin{array}{l}242 \\
203 \\
164 \\
275 \\
215\end{array}$ \\
\hline Mean..... & $\overline{17.6}$ & $\overline{33.8}$ & $\overline{2.31}$ & .33 & $\overline{272}$ & $\overline{232}$ \\
\hline \multicolumn{7}{|c|}{ October 1985} \\
\hline $\begin{array}{l}1 \ldots \ldots \\
2 \ldots \ldots \\
3 \ldots \ldots \\
4 \ldots \ldots \\
5 \ldots \ldots\end{array}$ & $\begin{array}{l}17.3 \\
16.6 \\
19.3 \\
20.7 \\
18.0\end{array}$ & $\begin{array}{l}39.9 \\
27.4 \\
25.4 \\
22.5 \\
24.1\end{array}$ & $\begin{array}{l}1.43 \\
1.91 \\
1.75 \\
1.86 \\
1.64\end{array}$ & $\begin{array}{r}0.32 \\
.24 \\
.32 \\
.35 \\
.30\end{array}$ & $\begin{array}{l}289 \\
233 \\
263 \\
229 \\
259\end{array}$ & $\begin{array}{l}230 \\
199 \\
238 \\
205 \\
237\end{array}$ \\
\hline $\begin{array}{c}6 \ldots \ldots \ldots \\
7 \ldots \ldots \\
8 \ldots \ldots \\
9 \ldots \ldots \\
10 \ldots \ldots\end{array}$ & $\begin{array}{r}19.6 \\
16.0 \\
10.4 \\
8.6 \\
11.5\end{array}$ & $\begin{array}{l}30.7 \\
47.4 \\
87.3 \\
80.7 \\
57.7\end{array}$ & $\begin{array}{l}3.99 \\
2.75 \\
1.31 \\
2.59 \\
2.98\end{array}$ & $\begin{array}{l}.22 \\
.31 \\
.38 \\
.24 \\
.27\end{array}$ & $\begin{array}{l}272 \\
270 \\
265 \\
233 \\
191\end{array}$ & $\begin{array}{l}241 \\
252 \\
231 \\
157 \\
153\end{array}$ \\
\hline $\begin{array}{l}11 \ldots \ldots \ldots \\
12 \ldots \ldots \\
13 \ldots \ldots \\
14 \ldots \ldots \\
15 \ldots \ldots\end{array}$ & $\begin{array}{l}10.0 \\
10.9 \\
13.0 \\
12.0 \\
12.8\end{array}$ & $\begin{array}{l}62.3 \\
52.9 \\
33.8 \\
22.6 \\
19.6\end{array}$ & $\begin{array}{l}1.75 \\
1.73 \\
3.90 \\
3.44 \\
2.77\end{array}$ & $\begin{array}{l}.47 \\
.33 \\
.23 \\
.29 \\
.24\end{array}$ & $\begin{array}{l}291 \\
240 \\
186 \\
201 \\
207\end{array}$ & $\begin{array}{l}214 \\
233 \\
132 \\
140 \\
185\end{array}$ \\
\hline $\begin{array}{l}16 \ldots \ldots \ldots \\
17 \ldots \ldots \ldots \\
18 \ldots \ldots \ldots \\
19 \ldots \ldots \ldots \\
20 \ldots \ldots\end{array}$ & $\begin{array}{l}10.7 \\
12.0 \\
11.9 \\
11.6 \\
13.3\end{array}$ & $\begin{array}{l}23.4 \\
25.9 \\
27.5 \\
35.7 \\
36.1\end{array}$ & $\begin{array}{l}1.19 \\
1.30 \\
1.37 \\
1.53 \\
2.32\end{array}$ & $\begin{array}{l}.29 \\
.44 \\
.43 \\
.35 \\
.26\end{array}$ & $\begin{array}{l}279 \\
280 \\
234 \\
259 \\
287\end{array}$ & $\begin{array}{l}221 \\
200 \\
209 \\
210 \\
224\end{array}$ \\
\hline $\begin{array}{l}21 \ldots \ldots \ldots \\
22 \ldots \ldots \ldots \\
23 \ldots \ldots \ldots \\
24 \ldots \ldots \ldots \\
25 \ldots \ldots \ldots\end{array}$ & $\begin{array}{r}\ldots \\
\overline{-.} \\
12.1 \\
12.6\end{array}$ & $\begin{array}{l}-- \\
45.5 \\
40.0 \\
42.5\end{array}$ & $\begin{array}{l}-- \\
1.30 \\
1.20 \\
1.34\end{array}$ & $\begin{array}{l}-- \\
-. \\
.42 \\
.39 \\
.41\end{array}$ & $\begin{array}{l}-- \\
261 \\
233 \\
263\end{array}$ & $\begin{array}{l}-- \\
197 \\
187 \\
194\end{array}$ \\
\hline $\begin{array}{l}26 \ldots \ldots \ldots \\
27 \ldots \ldots \ldots \\
28 \ldots \ldots \ldots \\
29 \ldots \ldots \\
30 \ldots \ldots\end{array}$ & $\begin{array}{l}12.7 \\
12.3 \\
11.7 \\
11.5 \\
11.2\end{array}$ & $\begin{array}{l}37.9 \\
35.2 \\
36.7 \\
37.2 \\
32.9\end{array}$ & $\begin{array}{l}1.24 \\
1.43 \\
1.17 \\
1.33 \\
1.72\end{array}$ & $\begin{array}{l}.38 \\
.45 \\
.53 \\
.31 \\
.30\end{array}$ & $\begin{array}{l}240 \\
277 \\
275 \\
243 \\
273\end{array}$ & $\begin{array}{l}190 \\
226 \\
187 \\
229 \\
228\end{array}$ \\
\hline $31 \ldots \ldots \ldots$ & 14.2 & 22.7 & 5.03 & .41 & 223 & 97 \\
\hline Mean..... & $\overline{13.2}$ & $\overline{38.4}$ & $\overline{2.04}$ & $\overline{.34}$ & $\overline{250}$ & $\overline{202}$ \\
\hline
\end{tabular}


Table 4. Meteorological data for undisturbed-land-surface site, May 1985 through June 1986-Continued

\begin{tabular}{|c|c|c|c|c|c|c|}
\hline \multirow{2}{*}{ Date } & \multirow{2}{*}{$\begin{array}{c}\text { Daily mean } \\
\text { air } \\
\text { temperature } \\
\text { (degrees } \\
\text { Celsius) }\end{array}$} & \multirow{2}{*}{$\begin{array}{l}\text { Daily } \\
\text { mean } \\
\text { relative } \\
\text { humidity } \\
\text { (percent) }\end{array}$} & \multicolumn{2}{|c|}{$\begin{array}{l}\text { Wind velocity } \\
\text { (meters per second) }\end{array}$} & \multicolumn{2}{|c|}{$\begin{array}{l}\text { Wind direction } \\
\text { (degrees) }\end{array}$} \\
\hline & & & $\begin{array}{l}\text { Daily } \\
\text { maximum }\end{array}$ & $\begin{array}{l}\text { Daily } \\
\text { mean }\end{array}$ & $\begin{array}{l}\text { Maximum } \\
\text { mean }\end{array}$ & $\begin{array}{l}\text { Daily } \\
\text { mean }\end{array}$ \\
\hline \multicolumn{7}{|c|}{ November 1985} \\
\hline $\begin{array}{l}1 \ldots \ldots \\
2 \ldots \ldots \\
3 \ldots \ldots \\
4 \ldots \ldots \\
5 \ldots \ldots\end{array}$ & $\begin{array}{l}-- \\
-- \\
-- \\
-- \\
14.1\end{array}$ & $\begin{array}{l}-- \\
-- \\
-- \\
-- \\
31.0\end{array}$ & $\begin{array}{l}-- \\
-- \\
-- \\
-- \\
2.22\end{array}$ & $\begin{array}{l}-- \\
-- \\
-- \\
-- \\
0.32\end{array}$ & $\begin{array}{l}-- \\
-- \\
-- \\
259\end{array}$ & $\begin{array}{l}-- \\
-- \\
-- \\
-- \\
189\end{array}$ \\
\hline $\begin{array}{c}6 \ldots \ldots \ldots \\
7 \ldots \ldots \ldots \\
8 \ldots \ldots \ldots \\
9 \ldots \ldots\end{array}$ & $\begin{array}{l}-- \\
9.3 \\
8.3 \\
7.1 \\
9.0\end{array}$ & $\begin{array}{l}-- \\
33.5 \\
38.2 \\
28.9 \\
34.6\end{array}$ & $\begin{array}{l}-- \\
1.85 \\
1.84 \\
2.84 \\
7.78\end{array}$ & $\begin{array}{l}- \\
.24 \\
.32 \\
.43 \\
.05\end{array}$ & $\begin{array}{l}-\overline{2} \\
286 \\
256 \\
311\end{array}$ & $\begin{array}{l}-\overline{-} \\
245 \\
236 \\
188 \\
313\end{array}$ \\
\hline $\begin{array}{l}11 \ldots \ldots \ldots \\
12 \ldots \ldots \\
13 \ldots \ldots \\
14 \ldots \ldots \\
15 \ldots \ldots\end{array}$ & $\begin{array}{r}2.6 \\
-1.6 \\
-4.8 \\
-1.1 \\
.3\end{array}$ & $\begin{array}{l}85.2 \\
94.1 \\
82.4 \\
77.2 \\
82.3\end{array}$ & $\begin{array}{r}3.92 \\
1.25 \\
2.27 \\
2.93 \\
.89\end{array}$ & $\begin{array}{l}.34 \\
.57 \\
.47 \\
.47 \\
.60\end{array}$ & $\begin{array}{l}258 \\
253 \\
217 \\
173 \\
239\end{array}$ & $\begin{array}{l}232 \\
253 \\
112 \\
127 \\
175\end{array}$ \\
\hline $\begin{array}{l}16 \ldots \ldots \ldots \\
17 \ldots \ldots \\
18 \ldots \ldots \\
19 \ldots \ldots \\
20 \ldots \ldots\end{array}$ & $\begin{array}{r}1.3 \\
2.3 \\
.6 \\
-3.1 \\
-2.8\end{array}$ & $\begin{array}{l}80.7 \\
73.9 \\
52.9 \\
49.4 \\
59.1\end{array}$ & $\begin{array}{l}.94 \\
1.42 \\
4.87 \\
1.85 \\
1.09\end{array}$ & $\begin{array}{l}.55 \\
.66 \\
.48 \\
.72 \\
.23\end{array}$ & $\begin{array}{l}225 \\
206 \\
144 \\
184 \\
259\end{array}$ & $\begin{array}{r}169 \\
185 \\
101 \\
92 \\
250\end{array}$ \\
\hline $\begin{array}{l}21 \ldots \ldots \ldots \\
22 \ldots \ldots \ldots \\
23 \ldots \ldots \ldots \\
24 \ldots \ldots \ldots \\
25 \ldots \ldots \ldots\end{array}$ & $\begin{array}{l}-.6 \\
-.1 \\
2.5 \\
5.9 \\
7.1\end{array}$ & $\begin{array}{l}60.1 \\
67.5 \\
71.2 \\
74.9 \\
--\end{array}$ & $\begin{array}{r}1.36 \\
1.54 \\
.81 \\
2.21 \\
.70\end{array}$ & $\begin{array}{l}.36 \\
.23 \\
.27 \\
.23 \\
.24\end{array}$ & $\begin{array}{l}259 \\
264 \\
282 \\
300 \\
263\end{array}$ & $\begin{array}{l}231 \\
224 \\
234 \\
240 \\
252\end{array}$ \\
\hline $\begin{array}{l}26 \ldots \ldots \ldots \\
27 \ldots \ldots \ldots \\
28 \ldots \ldots \ldots \\
29 \ldots \ldots \ldots \\
30 \ldots \ldots \ldots\end{array}$ & $\begin{array}{l}4.7 \\
4.5 \\
4.1 \\
5.9 \\
4.7\end{array}$ & $\begin{array}{l}-- \\
-- \\
-- \\
-- \\
64.4\end{array}$ & $\begin{array}{l}1.02 \\
1.45 \\
.95 \\
3.98 \\
2.38\end{array}$ & $\begin{array}{l}.47 \\
.27 \\
.52 \\
.22 \\
.51\end{array}$ & $\begin{array}{l}275 \\
268 \\
263 \\
296 \\
232\end{array}$ & $\begin{array}{l}244 \\
264 \\
229 \\
287 \\
121\end{array}$ \\
\hline Mean..... & $\overline{3.2}$ & $\overline{62.1}$ & $\overline{2.18}$ & .39 & $\overline{250}$ & $\overline{208}$ \\
\hline \multicolumn{7}{|c|}{ December 1985} \\
\hline $\begin{array}{l}1 \ldots \ldots \\
2 \ldots \ldots \\
3 \ldots \ldots \\
4 \ldots \ldots \\
5 \ldots \ldots\end{array}$ & $\begin{array}{l}3.2 \\
5.7 \\
6.6 \\
3.9 \\
4.3\end{array}$ & $\begin{array}{l}83.3 \\
97.5 \\
90.8 \\
87.2 \\
84.2\end{array}$ & $\begin{array}{r}1.05 \\
1.73 \\
1.06 \\
.75 \\
.99\end{array}$ & $\begin{array}{r}0.48 \\
.31 \\
.47 \\
.38 \\
.43\end{array}$ & $\begin{array}{l}274 \\
284 \\
252 \\
254 \\
270\end{array}$ & $\begin{array}{l}234 \\
268 \\
283 \\
192 \\
202\end{array}$ \\
\hline $\begin{array}{c}6 \ldots \ldots \ldots \\
7 \ldots \ldots \ldots \\
8 \ldots \ldots \ldots \\
9 \ldots \ldots\end{array}$ & $\begin{array}{l}3.6 \\
3.8 \\
3.7 \\
-- \\
--\end{array}$ & $\begin{array}{c}78.1 \\
77.3 \\
44.9 \\
-- \\
--\end{array}$ & $\begin{array}{l}1.06 \\
1.72 \\
4.86 \\
-- \\
--\end{array}$ & $\begin{array}{l}.31 \\
.40 \\
.66 \\
-. \\
--\end{array}$ & $\begin{array}{r}265 \\
282 \\
136 \\
-- \\
--\end{array}$ & $\begin{array}{r}232 \\
219 \\
91 \\
-- \\
--\end{array}$ \\
\hline $\begin{array}{l}11 \ldots \ldots \ldots \\
12 \ldots \ldots \\
13 \ldots \ldots \ldots \\
14 \ldots \ldots \ldots \\
15 \ldots \ldots \ldots\end{array}$ & $\begin{array}{r}-- \\
-1.0 \\
.8 \\
2.4\end{array}$ & $\begin{array}{c}-- \\
-- \\
49.0 \\
57.5 \\
52.3\end{array}$ & $\begin{array}{l}-- \\
-- \\
2.00 \\
1.24 \\
.92\end{array}$ & $\begin{array}{l}-- \\
-- \\
.34 \\
.36 \\
.44\end{array}$ & $\begin{array}{r}-- \\
-\overline{-} \\
208 \\
249 \\
255\end{array}$ & $\begin{array}{l}-- \\
-- \\
186 \\
225 \\
216\end{array}$ \\
\hline $\begin{array}{l}16 \ldots \ldots \ldots \\
17 \ldots \ldots \ldots \\
18 \ldots \ldots \ldots \\
19 \ldots \ldots \ldots \\
20 \ldots \ldots \ldots\end{array}$ & $\begin{array}{l}5.9 \\
6.9 \\
8.1 \\
4.6 \\
-.4\end{array}$ & $\begin{array}{l}37.3 \\
36.4 \\
38.0 \\
50.2 \\
63.2\end{array}$ & $\begin{array}{r}3.23 \\
3.41 \\
3.09 \\
1.19 \\
.81\end{array}$ & $\begin{array}{l}.34 \\
.50 \\
.32 \\
.39 \\
.43\end{array}$ & $\begin{array}{l}170 \\
158 \\
184 \\
250 \\
269\end{array}$ & $\begin{array}{l}126 \\
116 \\
146 \\
229 \\
143\end{array}$ \\
\hline
\end{tabular}


Table 4. Meteorological data for undisturbed-land-surface site, May 1985 through June $1986-$ Continued

\begin{tabular}{|c|c|c|c|c|c|c|}
\hline \multirow{2}{*}{ Date } & \multirow{2}{*}{$\begin{array}{c}\text { Daily mean } \\
\text { air } \\
\text { temperature } \\
\text { (degrees } \\
\text { Celsius) }\end{array}$} & \multirow{2}{*}{$\begin{array}{l}\text { Daily } \\
\text { mean } \\
\text { relative } \\
\text { humidity } \\
\text { (percent) }\end{array}$} & \multicolumn{2}{|c|}{$\begin{array}{l}\text { Wind velocity } \\
\text { (meters per second) }\end{array}$} & \multicolumn{2}{|c|}{$\begin{array}{l}\text { Wind direction } \\
\text { (degrees) }\end{array}$} \\
\hline & & & $\begin{array}{l}\text { Daily } \\
\text { maximum }\end{array}$ & $\begin{array}{l}\text { Daily } \\
\text { mean }\end{array}$ & $\begin{array}{l}\text { Maximum } \\
\text { mean }\end{array}$ & $\begin{array}{l}\text { Daily } \\
\text { mean }\end{array}$ \\
\hline \multicolumn{7}{|c|}{ December 1985 -Continued } \\
\hline $\begin{array}{l}21 \ldots \ldots \ldots \\
22 \ldots \ldots \ldots \\
23 \ldots \ldots \ldots \\
24 \ldots \ldots \ldots \\
25 \ldots \ldots \ldots\end{array}$ & $\begin{array}{l}-- \\
-- \\
5.7 \\
9.8 \\
9.4\end{array}$ & $\begin{array}{l}-- \\
-- \\
33.0 \\
32.2 \\
35.4\end{array}$ & $\begin{array}{l}-- \\
-- \\
1.92 \\
3.05 \\
2.66\end{array}$ & $\begin{array}{l}-- \\
-- \\
0.20 \\
.40 \\
.49\end{array}$ & $\begin{array}{l}-- \\
-- \\
233 \\
196 \\
201\end{array}$ & $\begin{array}{l}-- \\
-- \\
200 \\
191 \\
138\end{array}$ \\
\hline $\begin{array}{l}26 \ldots \ldots \ldots \\
27 \ldots \ldots \ldots \\
28 \ldots \ldots \ldots \\
29 \ldots \ldots \ldots \\
30 \ldots \ldots \ldots\end{array}$ & $\begin{array}{l}3.2 \\
2.3 \\
1.8 \\
4.5 \\
7.5\end{array}$ & $\begin{array}{l}51.3 \\
52.1 \\
52.6 \\
67.1 \\
75.4\end{array}$ & $\begin{array}{r}1.01 \\
.1 .08 \\
1.09 \\
.88 \\
1.31\end{array}$ & $\begin{array}{l}.35 \\
.53 \\
.63 \\
.36 \\
.42\end{array}$ & $\begin{array}{l}272 \\
257 \\
239 \\
260 \\
257\end{array}$ & $\begin{array}{l}234 \\
191 \\
201 \\
247 \\
247\end{array}$ \\
\hline $31 \ldots \ldots \ldots$ & 4.3 & 82.8 & .82 & .35 & 270 & 236 \\
\hline Mean...... & $\overline{4.4}$ & $\overline{60.4}$ & $\overline{1.72}$ & $\overline{.41}$ & $\overline{238}$ & $\overline{200}$ \\
\hline \multicolumn{7}{|c|}{ January 1986} \\
\hline $\begin{array}{l}1 \ldots \ldots \ldots \\
2 \ldots \ldots \\
3 \ldots \ldots \\
4 \ldots \ldots \\
5 \ldots \ldots\end{array}$ & $\begin{array}{l}2.4 \\
2.6 \\
3.9 \\
8.0 \\
7.2\end{array}$ & $\begin{array}{l}83.9 \\
80.4 \\
75.6 \\
61.0 \\
--\end{array}$ & $\begin{array}{r}1.32 \\
1.08 \\
1.01 \\
1.07 \\
.74\end{array}$ & $\begin{array}{r}0.34 \\
.45 \\
.47 \\
.39 \\
.58\end{array}$ & $\begin{array}{l}283 \\
251 \\
238 \\
284 \\
247\end{array}$ & $\begin{array}{l}198 \\
170 \\
202 \\
219 \\
201\end{array}$ \\
\hline $\begin{array}{c}6 \ldots \ldots \ldots \\
7 \ldots \ldots \ldots \\
8 \ldots \ldots \ldots \\
9 \ldots \ldots \ldots \\
10 \ldots \ldots\end{array}$ & $\begin{array}{r}5.5 \\
3.6 \\
.0 \\
3.8 \\
5.8\end{array}$ & $\begin{array}{l}61.3 \\
36.5 \\
54.2 \\
49.3 \\
46.2\end{array}$ & $\begin{array}{l}3.66 \\
4.06 \\
.87 \\
-- \\
1.01\end{array}$ & $\begin{array}{r}.38 \\
.08 \\
.48 \\
-. \\
.42\end{array}$ & $\begin{array}{r}190 \\
174 \\
247 \\
-- \\
243\end{array}$ & $\begin{array}{r}152 \\
156 \\
205 \\
-- \\
192\end{array}$ \\
\hline $\begin{array}{l}11 \ldots \ldots \ldots \\
12 \ldots \ldots \ldots \\
13 \ldots \ldots \\
14 \ldots \ldots \ldots \\
15 \ldots \ldots \ldots\end{array}$ & $\begin{array}{l}5.8 \\
5.4 \\
-- \\
4.8 \\
6.6\end{array}$ & $\begin{array}{c}52.5 \\
52.4 \\
-. \\
51.1 \\
68.4\end{array}$ & $\begin{array}{l}.94 \\
1.04 \\
1.34 \\
1.63\end{array}$ & $\begin{array}{r}.39 \\
.31 \\
-- \\
.35 \\
.38\end{array}$ & $\begin{array}{r}269 \\
260 \\
-- \\
232 \\
287\end{array}$ & $\begin{array}{r}230 \\
207 \\
-- \\
217 \\
249\end{array}$ \\
\hline $\begin{array}{l}16 \ldots \ldots \ldots \\
17 \ldots \ldots \ldots \\
18 \ldots \ldots \ldots \\
19 \ldots \ldots \ldots \\
20 \ldots \ldots \ldots\end{array}$ & $\begin{array}{l}3.6 \\
3.6 \\
7.1 \\
6.2 \\
8.0\end{array}$ & $\begin{array}{l}81.3 \\
77.8 \\
63.5 \\
59.1 \\
41.8\end{array}$ & $\begin{array}{l}1.21 \\
1.06 \\
1.18 \\
1.36 \\
2.92\end{array}$ & $\begin{array}{l}.53 \\
.59 \\
.47 \\
.44 \\
.54\end{array}$ & $\begin{array}{l}272 \\
281 \\
253 \\
244 \\
178\end{array}$ & $\begin{array}{l}208 \\
210 \\
174 \\
186 \\
149\end{array}$ \\
\hline $\begin{array}{l}21 \ldots \ldots \ldots \\
22 \ldots \ldots \ldots \\
23 \ldots \ldots \ldots \\
24 \ldots \ldots \ldots \\
25 \ldots \ldots \ldots\end{array}$ & $\begin{array}{l}-- \\
-- \\
-- \\
6.6 \\
6.6\end{array}$ & $\begin{array}{c}-- \\
-- \\
-- \\
33.7 \\
31.3\end{array}$ & $\begin{array}{l}-- \\
-- \\
-- \\
2.53 \\
2.37\end{array}$ & $\begin{array}{l}-- \\
-- \\
- \\
.39 \\
.38\end{array}$ & $\begin{array}{l}-- \\
-- \\
-- \\
176 \\
192\end{array}$ & $\begin{array}{l}-- \\
-- \\
-- \\
164 \\
165\end{array}$ \\
\hline $\begin{array}{l}26 \ldots \ldots \ldots \\
27 \ldots \ldots \ldots \\
28 \ldots \ldots \ldots \\
29 \ldots \ldots \\
30 \ldots \ldots\end{array}$ & $\begin{array}{l}4.8 \\
4.7 \\
5.1 \\
5.9 \\
7.9\end{array}$ & $\begin{array}{c}33.0 \\
34.7 \\
35.0 \\
36.4 \\
-.\end{array}$ & $\begin{array}{l}.94 \\
1.17 \\
1.54 \\
1.60 \\
1.74\end{array}$ & $\begin{array}{l}.23 \\
.32 \\
.29 \\
.34 \\
.35\end{array}$ & $\begin{array}{l}238 \\
228 \\
253 \\
276 \\
260\end{array}$ & $\begin{array}{l}210 \\
195 \\
220 \\
260 \\
248\end{array}$ \\
\hline $31 \ldots \ldots \ldots$ & 9.5 & 89.7 & 2.71 & .10 & 287 & 269 \\
\hline Mean..... & $\overline{5.4}$ & $\overline{55.6}$ & $\overline{1.62}$ & .38 & $\overline{244}$ & $\overline{202}$ \\
\hline
\end{tabular}


Table 4. Meteorological data for undisturbed-land-surface site, May 1985 through June 1986 -Continued

\begin{tabular}{|c|c|c|c|c|c|c|}
\hline \multirow{2}{*}{ Date } & \multirow{2}{*}{$\begin{array}{c}\text { Daily mean } \\
\text { air } \\
\text { temperature } \\
\text { (degrees } \\
\text { Celsius) }\end{array}$} & \multirow{2}{*}{$\begin{array}{l}\text { Daily } \\
\text { mean } \\
\text { relative } \\
\text { humidity } \\
\text { (percent) }\end{array}$} & \multicolumn{2}{|c|}{$\begin{array}{l}\text { Wind velocity } \\
\text { (meters per second) }\end{array}$} & \multicolumn{2}{|c|}{$\begin{array}{l}\text { Wind direction } \\
\text { (degrees) }\end{array}$} \\
\hline & & & $\begin{array}{l}\text { Daily } \\
\text { maximum }\end{array}$ & $\begin{array}{l}\text { Daily } \\
\text { mean }\end{array}$ & $\begin{array}{l}\text { Maximum } \\
\text { mean }\end{array}$ & $\begin{array}{l}\text { Daily } \\
\text { mean }\end{array}$ \\
\hline \multicolumn{7}{|c|}{ Fabruary 1986} \\
\hline $\begin{array}{l}1 \ldots \ldots \\
2 \ldots \ldots \\
3 \ldots \ldots \\
4 \ldots \ldots \\
5 \ldots \ldots\end{array}$ & $\begin{array}{l}7.5 \\
5.4 \\
4.4 \\
-- \\
--\end{array}$ & $\begin{array}{l}-- \\
81.4 \\
79.1 \\
-- \\
--\end{array}$ & $\begin{array}{l}1.05 \\
1.94 \\
1.61 \\
-- \\
--\end{array}$ & $\begin{array}{r}0.44 \\
.54 \\
.49 \\
-- \\
--\end{array}$ & $\begin{array}{l}249 \\
280 \\
297 \\
-- \\
--\end{array}$ & $\begin{array}{r}184 \\
248 \\
230 \\
-- \\
--\end{array}$ \\
\hline $\begin{array}{r}6 \ldots \ldots \ldots \\
7 \ldots \ldots \ldots \\
8 \ldots \ldots \ldots \\
9 \ldots \ldots \ldots \\
10 \ldots \ldots \ldots\end{array}$ & $\begin{array}{c}-- \\
-- \\
-- \\
-- \\
-1.2\end{array}$ & $\begin{array}{l}-- \\
-- \\
-- \\
-- \\
47.7\end{array}$ & $\begin{array}{l}-- \\
-- \\
-- \\
-- \\
2.39\end{array}$ & $\begin{array}{l}-- \\
-- \\
-- \\
-- \\
.20\end{array}$ & $\begin{array}{c}-- \\
-- \\
-- \\
-- \\
266\end{array}$ & $\begin{array}{c}-- \\
-- \\
-- \\
-- \\
246\end{array}$ \\
\hline $\begin{array}{l}11 \ldots \ldots \ldots \\
12 \ldots \ldots \ldots \\
13 \ldots \ldots \ldots \\
14 \ldots \ldots \ldots \\
15 \ldots \ldots \ldots\end{array}$ & $\begin{array}{l}-- \\
-- \\
8.5 \\
9.2 \\
9.3\end{array}$ & $\begin{array}{l}61.1 \\
74.7 \\
87.5 \\
88.1 \\
87.2\end{array}$ & $\begin{array}{l}1.64 \\
.81 \\
2.93 \\
2.13 \\
5.75\end{array}$ & $\begin{array}{l}.28 \\
.49 \\
.27 \\
.27 \\
.06\end{array}$ & $\begin{array}{l}269 \\
257 \\
307 \\
275 \\
309\end{array}$ & $\begin{array}{l}242 \\
248 \\
302 \\
278 \\
312\end{array}$ \\
\hline $\begin{array}{l}16 \ldots \ldots \ldots \\
17 \ldots \ldots \ldots \\
18 \ldots \ldots \ldots \\
19 \ldots \ldots \ldots \\
20 \ldots \ldots \ldots\end{array}$ & $\begin{array}{r}9.2 \\
9.5 \\
13.0 \\
12.8 \\
6.7\end{array}$ & $\begin{array}{l}75.9 \\
77.6 \\
70.9 \\
54.1 \\
55.5\end{array}$ & $\begin{array}{l}3.58 \\
3.11 \\
3.40 \\
6.93 \\
1.61\end{array}$ & $\begin{array}{l}.28 \\
.16 \\
.16 \\
.09 \\
.49\end{array}$ & $\begin{array}{l}315 \\
286 \\
303 \\
304 \\
281\end{array}$ & $\begin{array}{l}310 \\
296 \\
286 \\
310 \\
232\end{array}$ \\
\hline $\begin{array}{l}21 \ldots \ldots \ldots \\
22 \ldots \ldots \ldots \\
23 \ldots \ldots \ldots \\
24 \ldots \ldots \ldots \\
25 \ldots \ldots \ldots \ldots\end{array}$ & $\begin{array}{r}6.6 \\
6.6 \\
10.6 \\
10.2 \\
--\end{array}$ & $\begin{array}{l}53.5 \\
57.6 \\
57.0 \\
61.1 \\
--\end{array}$ & $\begin{array}{l}1.82 \\
1.41 \\
2.21 \\
2.37 \\
--\end{array}$ & $\begin{array}{l}.42 \\
.40 \\
.32 \\
.54 \\
--\end{array}$ & $\begin{array}{l}248 \\
264 \\
210 \\
220 \\
--\end{array}$ & $\begin{array}{c}202 \\
208 \\
171 \\
143 \\
--\end{array}$ \\
\hline $\begin{array}{l}26 \ldots \ldots \ldots \\
27 \ldots \ldots \ldots \\
28 \ldots \ldots \ldots\end{array}$ & $\begin{array}{l}14.1 \\
15.6 \\
13.3\end{array}$ & $\begin{array}{l}49.3 \\
36.1 \\
36.8\end{array}$ & $\begin{array}{l}1.21 \\
2.71 \\
1.47\end{array}$ & $\begin{array}{l}.32 \\
.38 \\
.49\end{array}$ & $\begin{array}{l}256 \\
216 \\
251\end{array}$ & $\begin{array}{l}224 \\
183 \\
210\end{array}$ \\
\hline Mean..... & 9.0 & $\overline{64.6}$ & $\overline{2.48}$ & .34 & $\overline{270}$ & $\overline{241}$ \\
\hline \multicolumn{7}{|c|}{ March 1986} \\
\hline $\begin{array}{l}1 \ldots \ldots \\
2 \ldots \ldots \\
3 \ldots \ldots \\
4 \ldots \ldots \\
5 \ldots \ldots\end{array}$ & $\begin{array}{l}12.7 \\
11.5 \\
13.7 \\
10.8 \\
--\end{array}$ & $\begin{array}{l}44.6 \\
59.8 \\
56.4 \\
43.4 \\
44.1\end{array}$ & $\begin{array}{l}1.61 \\
2.71 \\
2.17 \\
1.34 \\
1.28\end{array}$ & $\begin{array}{r}0.58 \\
.64 \\
.17 \\
.40 \\
.43\end{array}$ & $\begin{array}{l}263 \\
172 \\
225 \\
267 \\
272\end{array}$ & $\begin{array}{l}218 \\
134 \\
190 \\
228 \\
204\end{array}$ \\
\hline $\begin{array}{c}6 \ldots \ldots \ldots \\
7 \ldots \ldots \ldots \\
8 \ldots \ldots \ldots \\
9 \ldots \ldots \\
10 \ldots \ldots\end{array}$ & $\begin{array}{r}-- \\
11.3 \\
11.8 \\
8.2 \\
6.1\end{array}$ & $\begin{array}{l}45.5 \\
51.1 \\
65.8 \\
58.6 \\
91.2\end{array}$ & $\begin{array}{l}1.92 \\
1.93 \\
5.56 \\
3.49 \\
3.99\end{array}$ & $\begin{array}{l}.50 \\
.32 \\
.17 \\
.15 \\
.19\end{array}$ & $\begin{array}{l}267 \\
290 \\
302 \\
298 \\
291\end{array}$ & $\begin{array}{l}183 \\
256 \\
298 \\
280 \\
284\end{array}$ \\
\hline $\begin{array}{l}11 \ldots \ldots \ldots \\
12 \ldots \ldots \\
13 \ldots \ldots \\
14 \ldots \ldots \\
15 \ldots \ldots\end{array}$ & $\begin{array}{l}7.4 \\
5.7 \\
4.3 \\
5.6 \\
4.9\end{array}$ & $\begin{array}{l}68.9 \\
63.2 \\
64.9 \\
72.1 \\
73.9\end{array}$ & $\begin{array}{l}2.23 \\
2.90 \\
2.16 \\
1.48 \\
3.35\end{array}$ & $\begin{array}{l}.34 \\
.33 \\
.36 \\
.33 \\
.31\end{array}$ & $\begin{array}{l}277 \\
200 \\
282 \\
277 \\
281\end{array}$ & $\begin{array}{l}244 \\
188 \\
231 \\
244 \\
250\end{array}$ \\
\hline $\begin{array}{l}16 \ldots \ldots \ldots \\
17 \ldots \ldots \\
18 \ldots \ldots \\
19 \ldots \ldots \\
20 \ldots \ldots\end{array}$ & $\begin{array}{l}5.1 \\
-- \\
-- \\
-- \\
10.4\end{array}$ & $\begin{array}{c}85.0 \\
-- \\
-- \\
-- \\
54.9\end{array}$ & $\begin{array}{l}3.25 \\
-- \\
-- \\
-- \\
1.67\end{array}$ & $\begin{array}{l}.24 \\
-- \\
-- \\
.45\end{array}$ & $\begin{array}{r}287 \\
-- \\
-- \\
-- \\
241\end{array}$ & $\begin{array}{r}279 \\
-- \\
-- \\
-- \\
154\end{array}$ \\
\hline $\begin{array}{l}21 \ldots \ldots \ldots \\
22 \ldots \ldots \ldots \\
23 \ldots \ldots \\
24 \ldots \ldots \\
25 \ldots \ldots\end{array}$ & $\begin{array}{l}10.0 \\
10.6 \\
11.7 \\
13.6 \\
13.5\end{array}$ & $\begin{array}{l}54.9 \\
49.3 \\
46.2 \\
38.6 \\
42.2\end{array}$ & $\begin{array}{l}1.71 \\
1.88 \\
1.98 \\
2.42 \\
1.98\end{array}$ & $\begin{array}{l}.36 \\
.46 \\
.31 \\
.35 \\
.57\end{array}$ & $\begin{array}{l}246 \\
239 \\
263 \\
304 \\
250\end{array}$ & $\begin{array}{l}224 \\
208 \\
235 \\
266 \\
146\end{array}$ \\
\hline
\end{tabular}


Table 4. Meteorological data for undisturbed-land-surface site, May 1985 through June 1986 -Continued

\begin{tabular}{|c|c|c|c|c|c|c|}
\hline \multirow{2}{*}{ Date } & \multirow{2}{*}{$\begin{array}{c}\text { Daily mean } \\
\text { air } \\
\text { temperature } \\
\text { (degrees } \\
\text { Celsius) }\end{array}$} & \multirow{2}{*}{$\begin{array}{c}\text { Daily } \\
\text { mean } \\
\text { relative } \\
\text { humidity } \\
\text { (percent) }\end{array}$} & \multicolumn{2}{|c|}{$\begin{array}{l}\text { Wind velocity } \\
\text { (meters per second) }\end{array}$} & \multicolumn{2}{|c|}{$\begin{array}{l}\text { Wind direction } \\
\text { (degrees) }\end{array}$} \\
\hline & & & $\begin{array}{c}\text { Daily } \\
\text { maximum }\end{array}$ & $\begin{array}{l}\text { Daily } \\
\text { mean }\end{array}$ & $\begin{array}{l}\text { Maximum } \\
\text { mean }\end{array}$ & $\begin{array}{l}\text { Daily } \\
\text { mean }\end{array}$ \\
\hline \multicolumn{7}{|c|}{ March 1986-Continued } \\
\hline $\begin{array}{l}26 \ldots \ldots \ldots \\
27 \ldots \ldots \ldots \\
28 \ldots \ldots \ldots \\
29 \ldots \ldots \\
30 \ldots \ldots \ldots\end{array}$ & $\begin{array}{l}13.9 \\
14.2 \\
15.8 \\
17.5 \\
15.7\end{array}$ & $\begin{array}{l}46.8 \\
44.2 \\
42.3 \\
48.0 \\
50.8\end{array}$ & $\begin{array}{l}1.37 \\
1.46 \\
1.80 \\
1.88 \\
2.06\end{array}$ & $\begin{array}{l}0.38 \\
.36 \\
.30 \\
.50 \\
.50\end{array}$ & $\begin{array}{l}246 \\
259 \\
274 \\
243 \\
274\end{array}$ & $\begin{array}{l}220 \\
218 \\
214 \\
198 \\
206\end{array}$ \\
\hline $31 \ldots \ldots$ & 15.0 & 49.5 & 2.15 & .40 & 221 & 156 \\
\hline Mean..... & $\overline{10.8}$ & $\overline{55.6}$ & $\overline{2.28}$ & .37 & $\overline{261}$ & $\overline{220}$ \\
\hline \multicolumn{7}{|c|}{ Apr11 1986} \\
\hline $\begin{array}{l}1 \ldots \ldots \\
2 \ldots \ldots \\
3 \ldots \ldots \\
4 \ldots \ldots \\
5 \ldots \ldots\end{array}$ & $\begin{array}{r}16.0 \\
9.0 \\
11.6 \\
10.4 \\
11.6\end{array}$ & $\begin{array}{l}52.1 \\
29.7 \\
32.6 \\
41.5 \\
36.9\end{array}$ & $\begin{array}{l}4.21 \\
7.76 \\
3.14 \\
2.07 \\
3.32\end{array}$ & $\begin{array}{r}0.23 \\
.39 \\
.38 \\
.30 \\
.43\end{array}$ & $\begin{array}{l}286 \\
119 \\
218 \\
276 \\
250\end{array}$ & $\begin{array}{r}262 \\
60 \\
181 \\
210 \\
191\end{array}$ \\
\hline $\begin{array}{r}6 \ldots \ldots \ldots \\
7 \ldots \ldots \ldots \\
8 \ldots \ldots \ldots \\
9 \ldots \ldots \ldots \\
10 \ldots \ldots\end{array}$ & $\begin{array}{r}8.1 \\
9.5 \\
10.6 \\
12.2 \\
13.0\end{array}$ & $\begin{array}{l}64.1 \\
68.5 \\
62.2 \\
57.2 \\
50.8\end{array}$ & $\begin{array}{l}2.12 \\
2.57 \\
1.81 \\
1.24 \\
1.60\end{array}$ & $\begin{array}{l}.35 \\
.26 \\
.44 \\
.29 \\
.37\end{array}$ & $\begin{array}{l}250 \\
281 \\
296 \\
268 \\
273\end{array}$ & $\begin{array}{l}208 \\
265 \\
248 \\
266 \\
217\end{array}$ \\
\hline $\begin{array}{l}11 \ldots \ldots \ldots \\
12 \ldots \ldots \ldots \\
13 \ldots \ldots \ldots \\
14 \ldots \ldots \ldots \\
15 \ldots \ldots \ldots\end{array}$ & $\begin{array}{r}13.5 \\
13.2 \\
9.7 \\
10.9 \\
13.1\end{array}$ & $\begin{array}{l}44.0 \\
36.0 \\
25.6 \\
25.6 \\
30.8\end{array}$ & $\begin{array}{l}2.03 \\
5.67 \\
3.94 \\
2.13 \\
4.17\end{array}$ & $\begin{array}{l}.28 \\
.40 \\
.45 \\
.56 \\
.25\end{array}$ & $\begin{array}{l}264 \\
278 \\
169 \\
204 \\
260\end{array}$ & $\begin{array}{l}244 \\
215 \\
128 \\
156 \\
239\end{array}$ \\
\hline $\begin{array}{l}16 \ldots \ldots \ldots \\
17 \ldots \ldots \ldots \\
18 \ldots \ldots \ldots \\
19 \ldots \ldots \ldots \\
20 \ldots \ldots \ldots\end{array}$ & $\begin{array}{r}8.9 \\
9.1 \\
10.2 \\
12.1 \\
18.0\end{array}$ & $\begin{array}{l}53.6 \\
40.1 \\
30.7 \\
28.0 \\
31.0\end{array}$ & $\begin{array}{l}3.64 \\
2.74 \\
3.66 \\
1.84 \\
2.36\end{array}$ & $\begin{array}{l}.19 \\
.61 \\
.41 \\
.18 \\
.37\end{array}$ & $\begin{array}{l}286 \\
242 \\
178 \\
251 \\
242\end{array}$ & $\begin{array}{l}248 \\
145 \\
156 \\
209 \\
165\end{array}$ \\
\hline $\begin{array}{l}21 \ldots \ldots \ldots \\
22 \ldots \ldots \ldots \\
23 \ldots \ldots \ldots \\
24 \ldots \ldots \\
25 \ldots \ldots \ldots\end{array}$ & $\begin{array}{l}14.7 \\
18.2 \\
16.2 \\
15.4 \\
15.3\end{array}$ & $\begin{array}{l}44.8 \\
28.6 \\
28.6 \\
30.6 \\
27.8\end{array}$ & $\begin{array}{l}1.38 \\
2.96 \\
4.03 \\
2.72 \\
4.37\end{array}$ & $\begin{array}{l}.27 \\
.18 \\
.21 \\
.26 \\
.36\end{array}$ & $\begin{array}{l}250 \\
260 \\
261 \\
308 \\
258\end{array}$ & $\begin{array}{l}236 \\
251 \\
262 \\
287 \\
246\end{array}$ \\
\hline $\begin{array}{l}26 \ldots \ldots \ldots \\
27 \ldots \ldots \ldots \ldots \\
28 \ldots \ldots \ldots \\
29 \ldots \ldots \ldots \\
30 \ldots \ldots \ldots\end{array}$ & $\begin{array}{c}11.3 \\
13.0 \\
-- \\
-- \\
--\end{array}$ & $\begin{array}{l}25.5 \\
24.4 \\
24.6 \\
26.2 \\
27.0\end{array}$ & $\begin{array}{l}4.83 \\
2.05 \\
2.89 \\
2.06 \\
2.11\end{array}$ & $\begin{array}{l}.08 \\
.33 \\
.31 \\
.35 \\
.36\end{array}$ & $\begin{array}{l}202 \\
292 \\
303 \\
252 \\
276\end{array}$ & $\begin{array}{l}172 \\
243 \\
245 \\
227 \\
237\end{array}$ \\
\hline Mean..... & $\overline{12.4}$ & $\overline{37.6}$ & $\overline{3.05}$ & .33 & $\overline{252}$ & $\overline{214}$ \\
\hline \multicolumn{7}{|c|}{ May 1986} \\
\hline $\begin{array}{l}1 \ldots \ldots \ldots \\
2 \ldots \ldots \\
3 \ldots \ldots \\
4 \ldots \ldots \\
5 \ldots \ldots\end{array}$ & $\begin{array}{l}18.8 \\
19.0 \\
13.5 \\
12.6\end{array}$ & $\begin{array}{l}22.2 \\
23.7 \\
28.2 \\
30.9 \\
25.5\end{array}$ & $\begin{array}{l}2.85 \\
3.28 \\
5.66 \\
3.86 \\
3.23\end{array}$ & $\begin{array}{r}0.36 \\
.32 \\
.12 \\
.49 \\
.13\end{array}$ & $\begin{array}{l}286 \\
260 \\
304 \\
240 \\
294\end{array}$ & $\begin{array}{r}251 \\
224 \\
-- \\
176 \\
286\end{array}$ \\
\hline $\begin{array}{c}6 \ldots \ldots \ldots \\
7 \ldots \ldots \ldots \\
8 \ldots \ldots \ldots \\
9 \ldots \ldots \\
10 \ldots \ldots\end{array}$ & $\begin{array}{l}7.9 \\
10.8 \\
12.1 \\
13.7 \\
17.1\end{array}$ & $\begin{array}{l}65.4 \\
46.9 \\
39.2 \\
33.5 \\
25.8\end{array}$ & $\begin{array}{l}2.78 \\
4.01 \\
3.02 \\
2.28 \\
4.87\end{array}$ & $\begin{array}{l}.25 \\
.41 \\
.51 \\
.36 \\
.20\end{array}$ & $\begin{array}{l}278 \\
182 \\
231 \\
267 \\
306\end{array}$ & $\begin{array}{l}250 \\
152 \\
127 \\
158 \\
254\end{array}$ \\
\hline
\end{tabular}


Table 4. Meteorological data for undisturbed-land-surface site, May 1985 through June 1986-Continued

\begin{tabular}{|c|c|c|c|c|c|c|}
\hline \multirow{2}{*}{ Date } & \multirow{2}{*}{$\begin{array}{c}\text { Daily mean } \\
\text { air } \\
\text { temperature } \\
\text { (degrees } \\
\text { Celsius) }\end{array}$} & \multirow{2}{*}{$\begin{array}{l}\text { Daily } \\
\text { mean } \\
\text { relative } \\
\text { humidity } \\
\text { (percent) }\end{array}$} & \multicolumn{2}{|c|}{$\begin{array}{l}\text { Wind velocity } \\
\text { (meters per second) }\end{array}$} & \multicolumn{2}{|c|}{$\begin{array}{l}\text { Wind direction } \\
\text { (degrees) }\end{array}$} \\
\hline & & & $\begin{array}{c}\text { Daily } \\
\text { maximum }\end{array}$ & $\begin{array}{l}\text { Daily } \\
\text { mean }\end{array}$ & $\begin{array}{l}\text { Maximum } \\
\text { mean }\end{array}$ & $\begin{array}{l}\text { Daily } \\
\text { mean }\end{array}$ \\
\hline \multicolumn{7}{|c|}{ May 1986--Continued } \\
\hline $\begin{array}{l}11 \ldots \ldots \ldots \\
12 \ldots \ldots \ldots \\
13 \ldots \ldots \\
14 \ldots \ldots \ldots \\
15 \ldots \ldots\end{array}$ & $\begin{array}{l}16.3 \\
17.2 \\
19.1 \\
20.1 \\
22.1\end{array}$ & $\begin{array}{l}30.9 \\
24.9 \\
21.3 \\
21.9 \\
21.0\end{array}$ & $\begin{array}{l}2.61 \\
2.29 \\
1.99 \\
2.40 \\
3.70\end{array}$ & $\begin{array}{l}0.16 \\
.35 \\
.23 \\
.29 \\
.31\end{array}$ & $\begin{array}{l}275 \\
245 \\
270 \\
276 \\
182\end{array}$ & $\begin{array}{l}262 \\
204 \\
261 \\
232 \\
157\end{array}$ \\
\hline $\begin{array}{l}16 \ldots \ldots \ldots \\
17 \ldots \ldots \ldots \\
18 \ldots \ldots \ldots \\
19 \ldots \ldots\end{array}$ & $\begin{array}{c}20.0 \\
17.9 \\
18.9 \\
--\end{array}$ & $\begin{array}{l}15.9 \\
16.8 \\
17.0 \\
--\end{array}$ & $\begin{array}{l}4.04 \\
2.54 \\
1.66 \\
--\end{array}$ & $\begin{array}{r}.40 \\
.18 \\
.39 \\
-.\end{array}$ & $\begin{array}{l}158 \\
229 \\
284 \\
--\end{array}$ & $\begin{array}{l}115 \\
213 \\
209 \\
--\end{array}$ \\
\hline $20 \ldots \ldots \ldots$ & 22.8 & 20.0 & 4.13 & .32 & 287 & 244 \\
\hline $\begin{array}{l}21 \ldots \ldots \cdots \\
22 \ldots \ldots \cdots \\
23 \ldots \ldots \cdots \\
24 \ldots \ldots \\
25 \ldots \ldots \\
\end{array}$ & $\begin{array}{l}21.7 \\
17.5 \\
17.8 \\
20.8 \\
22.8\end{array}$ & $\begin{array}{l}21.0 \\
14.3 \\
16.0 \\
14.6 \\
15.2\end{array}$ & $\begin{array}{l}5.47 \\
3.24 \\
1.84 \\
1.45 \\
1.50\end{array}$ & $\begin{array}{l}.20 \\
.31 \\
.65 \\
.56 \\
.44\end{array}$ & $\begin{array}{l}292 \\
218 \\
216 \\
279 \\
287\end{array}$ & $\begin{array}{l}274 \\
228 \\
221 \\
226 \\
187\end{array}$ \\
\hline $\begin{array}{l}26 \ldots \ldots \ldots \\
27 \ldots \ldots \ldots \\
28 \ldots \ldots \ldots \\
29 \ldots \ldots \cdots \\
30 \ldots \ldots\end{array}$ & $\begin{array}{l}24.1 \\
24.9 \\
25.7 \\
26.1 \\
24.7\end{array}$ & $\begin{array}{l}15.9 \\
15.6 \\
20.0 \\
24.1 \\
22.1\end{array}$ & $\begin{array}{l}2.03 \\
2.02 \\
2.53 \\
1.75 \\
1.89\end{array}$ & $\begin{array}{l}.33 \\
.33 \\
.40 \\
.52 \\
.37\end{array}$ & $\begin{array}{l}282 \\
247 \\
250 \\
286 \\
230\end{array}$ & $\begin{array}{l}359 \\
225 \\
224 \\
212 \\
180\end{array}$ \\
\hline $31 \ldots \ldots \ldots$ & -- & -- & -- & -- & -- & -- \\
\hline Mean..... & 18.8 & 24.5 & $\overline{2.93}$ & .34 & $\overline{257}$ & $\overline{218}$ \\
\hline \multicolumn{7}{|c|}{ June 1986} \\
\hline $\begin{array}{l}1 \ldots \ldots \ldots \\
2 \ldots \ldots \ldots \\
3 \ldots \ldots \cdots \\
4 \ldots \ldots \\
5 \ldots \ldots \ldots\end{array}$ & $\begin{array}{l}29.1 \\
28.2 \\
26.9 \\
26.1 \\
24.5\end{array}$ & $\begin{array}{l}22.7 \\
25.5 \\
26.0 \\
19.3 \\
16.6\end{array}$ & $\begin{array}{l}2.28 \\
2.76 \\
2.41 \\
3.10 \\
3.56\end{array}$ & $\begin{array}{l}0.34 \\
.23 \\
.47 \\
.27 \\
.26\end{array}$ & $\begin{array}{l}298 \\
278 \\
272 \\
266 \\
297\end{array}$ & $\begin{array}{l}220 \\
245 \\
215 \\
260 \\
265\end{array}$ \\
\hline $\begin{array}{c}6 \ldots \ldots \ldots \\
7 \ldots \ldots \\
8 \ldots \ldots \\
9 \ldots \ldots \\
10 \ldots \ldots\end{array}$ & $\begin{array}{l}21.4 \\
21.9 \\
23.5 \\
23.2 \\
23.0\end{array}$ & $\begin{array}{l}20.3 \\
21.0 \\
24.1 \\
17.3 \\
21.2\end{array}$ & $\begin{array}{l}2.47 \\
3.28 \\
4.48 \\
2.96 \\
1.98\end{array}$ & $\begin{array}{l}.35 \\
.39 \\
.26 \\
.13 \\
.42\end{array}$ & $\begin{array}{l}272 \\
248 \\
195 \\
206 \\
234\end{array}$ & $\begin{array}{l}220 \\
236 \\
117 \\
185 \\
199\end{array}$ \\
\hline $\begin{array}{l}11 \ldots \ldots \cdots \\
12 \ldots \ldots \cdots \\
13 \ldots \ldots \cdots \\
14 \ldots \ldots \\
15 \ldots \ldots \cdots\end{array}$ & $\begin{array}{l}23.6 \\
23.9 \\
23.7 \\
24.5 \\
--\end{array}$ & $\begin{array}{l}18.8 \\
15.3 \\
13.2 \\
14.3 \\
16.2\end{array}$ & $\begin{array}{l}1.72 \\
2.49 \\
1.80 \\
3.00 \\
2.70\end{array}$ & $\begin{array}{l}.37 \\
.33 \\
.52 \\
.40 \\
.29\end{array}$ & $\begin{array}{l}274 \\
279 \\
267 \\
283 \\
273\end{array}$ & $\begin{array}{l}233 \\
248 \\
224 \\
210 \\
226\end{array}$ \\
\hline $\begin{array}{l}16 \ldots \ldots \ldots \\
17 \ldots \ldots \cdots \\
18 \ldots \ldots \cdots \\
19 \ldots \ldots \cdots \\
20 \ldots \ldots \cdots\end{array}$ & $\begin{array}{l}-- \\
24.3 \\
23.9 \\
23.6 \\
22.6\end{array}$ & $\begin{array}{l}-- \\
15.6 \\
15.3 \\
14.5 \\
12.1\end{array}$ & $\begin{array}{l}-2 . \\
3.20 \\
2.86 \\
2.23 \\
2.41\end{array}$ & $\begin{array}{l}-- \\
.22 \\
.24 \\
.33 \\
.23\end{array}$ & $\begin{array}{l}-- \\
262 \\
284 \\
260 \\
284\end{array}$ & $\begin{array}{l}-- \\
247 \\
256 \\
240 \\
246\end{array}$ \\
\hline $\begin{array}{l}21 \ldots \ldots \cdots \\
22 \ldots \ldots \cdots \\
23 \ldots \ldots \cdots \\
24 \ldots \ldots \cdots \\
25 \ldots \ldots\end{array}$ & $\begin{array}{l}23.2 \\
24.5 \\
25.7 \\
27.2 \\
29.1\end{array}$ & $\begin{array}{l}12.0 \\
10.9 \\
13.9 \\
19.2 \\
15.5\end{array}$ & $\begin{array}{l}1.68 \\
1.55 \\
2.47 \\
2.49 \\
2.59\end{array}$ & $\begin{array}{l}.37 \\
.37 \\
.33 \\
.40 \\
.23\end{array}$ & $\begin{array}{l}263 \\
253 \\
276 \\
258 \\
290\end{array}$ & $\begin{array}{l}223 \\
224 \\
205 \\
252 \\
254\end{array}$ \\
\hline $\begin{array}{l}26 \ldots \ldots \ldots \\
27 \ldots \ldots \ldots \\
28 \ldots \ldots \\
29 \ldots \ldots \ldots \\
30 \ldots \ldots \\
\end{array}$ & $\begin{array}{l}28.0 \\
27.8 \\
28.4 \\
28.0 \\
--\end{array}$ & $\begin{array}{l}13.4 \\
14.4 \\
18.7 \\
18.7 \\
--\end{array}$ & $\begin{array}{l}2.84 \\
3.51 \\
3.73 \\
3.64 \\
--\end{array}$ & $\begin{array}{r}.30 \\
.17 \\
.31 \\
.14 \\
-.\end{array}$ & $\begin{array}{l}266 \\
259 \\
294 \\
307 \\
--\end{array}$ & $\begin{array}{l}239 \\
251 \\
274 \\
284 \\
--\end{array}$ \\
\hline Mean..... & $\overline{25.2}$ & $\overline{17.4}$ & $\overline{2.72}$ & .31 & $\overline{268}$ & $\overline{232}$ \\
\hline
\end{tabular}

